\title{
The Low-Energy Telescope (LET) and SEP Central Electronics for the STEREO Mission
}

\author{
R.A. Mewaldt • C.M.S. Cohen · W.R. Cook • A.C. Cummings • A.J. Davis • S. Geier • \\ B. Kecman • J. Klemic • A.W. Labrador · R.A. Leske · H. Miyasaka • V. Nguyen • \\ R.C. Ogliore · E.C. Stone · R.G. Radocinski - M.E. Wiedenbeck • J. Hawk • \\ S. Shuman $\cdot$ T.T. von Rosenvinge $\cdot$ K. Wortman
}

Received: 5 January 2007 / Accepted: 15 October 2007 / Published online: 12 December 2007

(C) Springer Science+Business Media B.V. 2007

\begin{abstract}
The Low-Energy Telescope (LET) is one of four sensors that make up the Solar Energetic Particle (SEP) instrument of the IMPACT investigation for NASA's STEREO mission. The LET is designed to measure the elemental composition, energy spectra, angular distributions, and arrival times of $\mathrm{H}$ to $\mathrm{Ni}$ ions over the energy range from $\sim 3$ to $\sim 30 \mathrm{MeV} /$ nucleon. It will also identify the rare isotope ${ }^{3} \mathrm{He}$ and trans-iron nuclei with $30 \leq Z \leq 83$. The SEP measurements from the two STEREO spacecraft will be combined with data from ACE and other 1-AU spacecraft to provide multipoint investigations of the energetic particles that result from interplanetary shocks driven by coronal mass ejections (CMEs) and from solar flare events. The multipoint in situ observations of SEPs and solarwind plasma will complement STEREO images of CMEs in order to investigate their role in space weather. Each LET instrument includes a sensor system made up of an array of 14 solid-state detectors composed of 54 segments that are individually analyzed by custom Pulse Height Analysis System Integrated Circuits (PHASICs). The signals from four PHASIC chips in each LET are used by a Minimal Instruction Set Computer (MISC) to provide onboard particle identification of a dozen species in $\sim 12$ energy intervals at event rates of $\sim 1,000$ events/sec. An additional control unit, called SEP Central, gathers data from the four SEP sensors, controls the SEP bias supply, and manages the interfaces to the sensors and the SEP interface to the Instrument Data Processing Unit (IDPU). This article outlines the scientific objectives that LET will address, describes the design and operation of LET and the SEP Central electronics, and discusses the data products that will result.
\end{abstract}

\footnotetext{
R.A. Mewaldt $(\varangle)$ - C.M.S. Cohen · W.R. Cook · A.C. Cummings · A.J. Davis · S. Geier · B. Kecman •

J. Klemic · A.W. Labrador · R.A. Leske · H. Miyasaka · V. Nguyen · R.C. Ogliore - E.C. Stone

California Institute of Technology, Pasadena, CA 91125, USA

e-mail: rmewaldt@srl.caltech.edu
}

R.G. Radocinski · M.E. Wiedenbeck

Jet Propulsion Laboratory, California Institute of Technology, Pasadena, CA 91109, USA

J. Hawk · S. Shuman · T.T. von Rosenvinge $\cdot$ K. Wortman

NASA/Goddard Space Flight Center, Greenbelt, MD 20771, USA 
Keywords Space vehicles: instruments - Instrumentation: detectors - Sun: particle emission - Sun: coronal mass ejections - Sun: flares $\cdot$ Acceleration of particles

\section{Acronyms and Abbreviations}

ACE Advanced Composition Explorer

ACR Anomalous Cosmic Ray

ADC Analog-to-Digital Converter

ALU Arithmetic Logic Unit

ApID Application process Identifier

ASCII American Standard Code for Information Interchange

ASIC Application Specific Integrated Circuit

C\&DH Command and Data Handling

CCSDS Consultative Committee for Space Data Systems

CF Correction Factor

CIR Corotating Interaction Region

CME Coronal Mass Ejection

CMOS Complementary Metal Oxide Semiconductor

CNO Carbon, Nitrogen, and Oxygen element group

CPU Central Processing Unit

CPU24 GSFC version of MISC

CRIS Cosmic Ray Isotope Spectrometer

CV Capacitance (C) vs. Voltage (V)

DAC Digital-to-Analog Converter

DC Direct Current

DPU Data Processing Unit

EEPROM Electronically Erasable Programmable Read-Only Memory

EGSE Electrical Ground Support Equipment

EM Engineering Model

EOR End of Record

EPAM Electron, Proton, and Alpha Monitor

EPHIN Electron Proton Helium Instrument (SoHO)

ERH Event Record Header

ESA European Space Agency

ESP Energetic Storm Particles

EUV Extreme Ultraviolet

E/M Energy/nucleon

FET Field Effect Transistor

FM1 Flight Model 1

FM2 Flight Model 2

FOV Field Of View

FPGA Field-Programmable Gate Array

FR4 Flame Resistant 4 (printed circuit board material)

F.S. Full scale

GALEX Galaxy Evolution Explorer

GCR Galactic Cosmic Ray

GEANT Geometry And Tracking (A toolkit for the simulation of particles through matter)

GOES Geostationary Operational Environmental Satellite

GSE Ground Support Equipment 


\begin{tabular}{|c|c|}
\hline GSFC & Goddard Space Flight Center \\
\hline HAZ & "HAZard" event \\
\hline HET & High Energy Telescope \\
\hline HV & High Voltage \\
\hline HVPS & High Voltage Power Supply \\
\hline $\mathrm{I} / \mathrm{F}$ & Interface \\
\hline $\mathrm{I} / \mathrm{O}$ & Input/Output \\
\hline ICD & Interface Control Document \\
\hline ICME & Interplanetary Coronal Mass Ejection \\
\hline ID & Identification \\
\hline IDPU & IMPACT Data Processing Unit \\
\hline IMF & Interplanetary Magnetic Field \\
\hline IMP & Interplanetary Monitoring Platform \\
\hline IMPACT & In situ Measurements of Particles And CME Transients \\
\hline ISEE-3 & International Sun-Earth Explorer 3 \\
\hline ISM & Interstellar Medium \\
\hline IT & Information Technology \\
\hline ITAR & International Traffic in Arms Regulations \\
\hline ITO & Indium Tin Oxide \\
\hline IV & Leakage current (I) vs. Voltage (V) \\
\hline JPL & Jet Propulsion Laboratory \\
\hline LBL & Lawrence Berkeley Laboratory \\
\hline LEMT & Low Energy Matrix Telescope \\
\hline LET & Low Energy Telescope \\
\hline $\mathrm{LiBeB}$ & Lithium, Beryllium, and Boron element group \\
\hline LVPS & Low Voltage Power Supply \\
\hline MAG & Magnetometer \\
\hline MISC & Minimal Instruction Set Computer \\
\hline MRD & Mission Requirements Document \\
\hline MSU & Michigan State University \\
\hline NASA & National Aeronautics and Space Administration \\
\hline NASTRAN & NASA Structural Analysis system \\
\hline $\mathrm{NeMgSi}$ & Neon, Magnesium, and Silicon element group \\
\hline NOAA & National Oceanic and Atmospheric Administration \\
\hline NSCL & National Superconducting Cyclotron Laboratory \\
\hline OGO & Orbiting Geophysical Observatory \\
\hline PDFE & Particle Detector Front End \\
\hline PEN & Penetrating event \\
\hline PHA & Pulse Height Analyzer \\
\hline PHASIC & Pulse Height Analysis System Integrated Circuit \\
\hline PLASTIC & Plasma And Suprathermal Ion Composition \\
\hline PSI & Pounds per Square Inch \\
\hline RHESSI & Ramaty High Energy Solar Spectroscopic Imager \\
\hline RISC & Reduced Instruction Set Computer \\
\hline RTSW & Real-Time Solar Wind \\
\hline SAMPEX & Solar, Anomalous, and Magnetospheric Particle Explorer \\
\hline SDO & Solar Dynamics Observatory \\
\hline SECCHI & Sun Earth Connection Coronal and Heliospheric Investigation \\
\hline SEP & Solar Energetic Particle \\
\hline
\end{tabular}




$\begin{array}{ll}\text { SEPT } & \text { Solar Electron Proton Telescope } \\ \text { SEPT-E } & \text { Ecliptic-viewing component of SEPT } \\ \text { SEPT-NS } & \text { North/South viewing component of SEPT } \\ \text { SIS } & \text { Solar Isotope Spectrometer } \\ \text { SIT } & \text { Suprathermal Ion Telescope } \\ \text { SOHO } & \text { Solar Heliospheric Observatory } \\ \text { SRAM } & \text { Static Random Access Memory } \\ \text { SRL } & \text { Space Radiation Laboratory } \\ \text { SSD } & \text { Solid-State Detector } \\ \text { STEREO } & \text { Solar Terrestrial Relations Observatory } \\ \text { STIM } & \text { Stimulated (pulser-produced) event } \\ \text { SWAVES } & \text { STEREO/WAVES Radio and Plasma Wave Experiment } \\ \text { TCP/IP } & \text { Transmission Control Protocol/Internet Protocol } \\ \text { TOF } & \text { Time Of Flight } \\ \text { UCB } & \text { University of California Berkeley } \\ \text { UH } & \text { Ultra-Heavy } \\ \text { ULEIS } & \text { Ultra-Low Energy Isotope Spectrometer } \\ \text { UT } & \text { Universal Time } \\ \text { VLSI } & \text { Very Large Scale Integration }\end{array}$

\section{Introduction}

The Solar Terrestrial Relations Observatory (STEREO) will employ two nearly identical spacecraft in orbit about the Sun (one moving ahead of the Earth, and one moving behind) to provide the first-ever 3-D images of coronal mass ejections (CMEs). These stereo images will be supplemented by multipoint in situ measurements of solar wind and CME plasma and the energetic particles accelerated in association with solar eruptions, and by multipoint observations of radio bursts occurring in these events. The Low Energy Telescope (LET) is one of four solar energetic particle sensors for the IMPACT (In Situ Measurements of Particles and CME Transients) investigation on STEREO. IMPACT provides measurements of solar wind and suprathermal electrons, interplanetary magnetic fields, and solar energetic particles (see Luhmann et al., 2005, 2007). The Solar Energetic Particle (SEP) suite is composed of the Solar Electron Proton Telescope (SEPT; Müller-Mellin et al. 2007), the Suprathermal Ion Telescope (SIT; Mason et al. 2007), the LET, and the High Energy Telescope (HET; von Rosenvinge et al. 2007).

The STEREO Mission Requirements Document (MRD) includes the following science objective that is specifically directed toward energetic particle studies:

Discover the mechanisms and sites of energetic particle acceleration in the low corona and the interplanetary medium

This leads to two additional, more specific objectives:

- Characterize distribution functions to an accuracy of $\pm 10 \%$ for electrons and/or ions with energies typical of solar energetic particle populations.

- Determine the location of particle acceleration in the low corona to within $300,000 \mathrm{~km}$ in radius and in interplanetary space to within $20^{\circ}$ in total longitude.

The four SEP instruments complement each other in addressing these objectives by providing comprehensive measurements of the composition and energy spectra of energetic nuclei from $\mathrm{H}$ to $\mathrm{Ni}(1 \leq Z \leq 28)$ spanning the energy range from $\sim 0.03$ to 
Fig. 1 Species and energy coverage of the four SEP sensors. The HET coverage is shown in $r e d$, the LET coverage in blue, the SIT coverage in black, and the SEPT coverage in green. The SIT and LET sensors can measure trans-iron species with $Z>30$

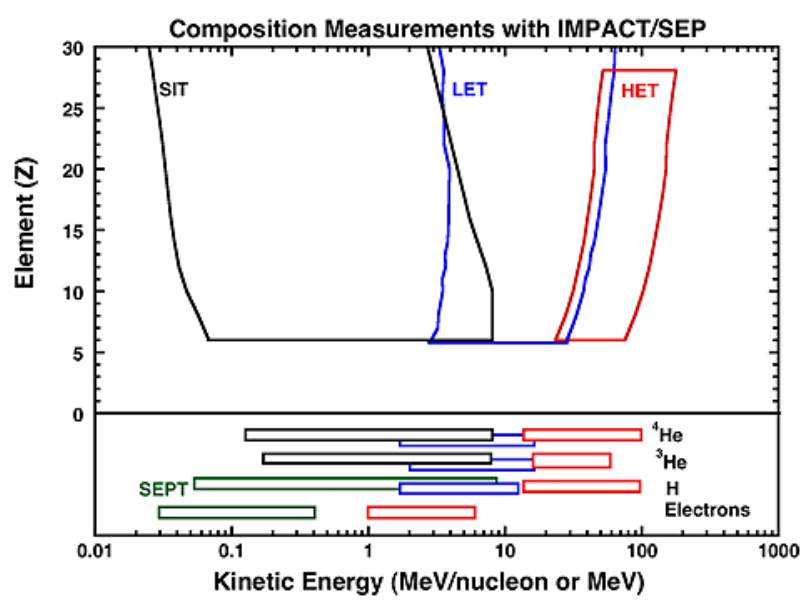

$>100 \mathrm{MeV} /$ nucleon, as well as electrons from 0.03 to $6 \mathrm{MeV}$ (see Fig. 1). In addition, the SIT and LET instruments will be sensitive to trans-iron nuclei with $30 \leq Z \leq 83$, and SEPT and LET will provide information on the pitch-angle distributions of solar energetic particles.

A block diagram of the SEP system is shown in Fig. 2. The LET, HET, and SIT sensors each include a dedicated microprocessor for onboard data processing and sensor control. An additional control unit, called SEP Central, gathers data from the four SEP sensors, controls the SEP bias supply, and manages the interfaces to the sensors and the SEP interface to the Instrument Data Processing Unit (IDPU). A photo of LET, HET, and SEP Central is shown in Fig. 3.

In this article we describe the LET instrument, including the science objectives, design requirements, measurement capabilities, and data products. The paper documents the features and capabilities of LET, as well as the onboard software and functions that can be altered by command or with new table uploads. There are two identical LET instruments: Flight Model 1 (FM1) is flying on the STEREO Ahead spacecraft, and FM2 is on the Behind spacecraft. Table 1 summarizes key characteristics of the LET instrument, and refers to the sections, figures, and tables where more details can be found. It is intended that sufficient information be provided so that a broad range of users in the solar and space physics community can make use of LET data.

The primary measurement goal of LET is to measure the composition, energy spectra, and time variations of solar energetic particles ranging from $\mathrm{H}$ to Ni. The energy range for oxygen extends from $\sim 3$ to $30 \mathrm{MeV} /$ nucleon (Fig. 1). In this range the intensities can vary by a factor of $\sim 10^{6}$, as illustrated in Fig. 4 , which includes data from 9 years of the 11-year solar cycle. During the last two years of solar minimum (1997-1998) there were not many SEP events, and the daily intensities typically reflect the quiet-time intensities of anomalous cosmic ray (ACR) and galactic cosmic ray (GCR) oxygen nuclei. Typical quiet time energy spectra from the last solar minimum are shown in Fig. 5. Superimposed on the quiet time intensities in Fig. 4 are hundreds of SEP events that typically last a few days. The frequency of large SEP events began to pick up in 1997-1998 and continued through 2005. Based on the $>30 \mathrm{MeV}$ proton fluences measured by NOAA's GOES satellites, the two largest events were the July 14, 2000 (Bastille Day), event and the October 28, 2003, event. Figure 5 includes an oxygen spectrum from the January 20, 2005 event, which had a powerlaw spectrum extending over two decades in energy. 


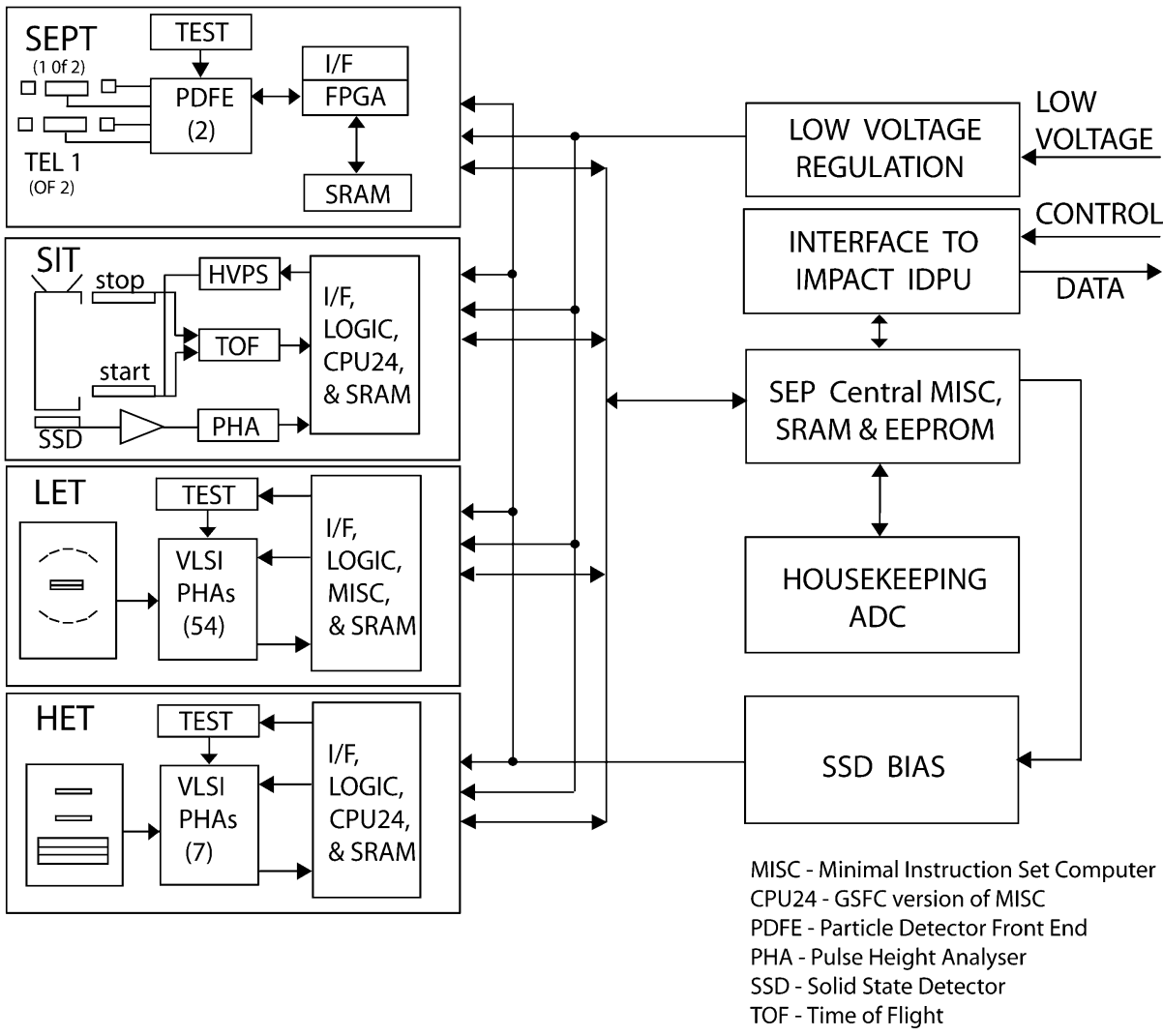

Fig. 2 SEP Sensor Suite block diagram. LET, HET, and SIT each include a dedicated microprocessor. An additional microprocessor, SEP Central, gathers data from the four SEP sensors, controls the SEP bias supply, and manages the interfaces to the sensors and the SEP interface to the Instrument Data Processing Unit (IDPU)

STEREO was launched on October 25, 2006, during the approach to solar minimum, which should be reached sometime in 2007. The frequency of SEP events is expected to begin to increase in 2008 .

\section{Science Objectives}

A key aspect of the STEREO mission is the combination of stereo imaging of solar eruptions and CMEs (see Howard et al. 2007) with multipoint in situ measurements of solar wind and CME plasma (Galvin et al. 2007; Lin et al. 2007) and the energetic particles accelerated in association with these events. There will also be multipoint observations of the radio bursts associated with solar eruptions (Bougeret et al. 2007). In addition, near-Earth instruments will provide a third imaging point of view (RHESSI, SoHO, Hinode, SDO, TRACE, and ground-based observations) as well as a third in situ point of view (ACE, Wind, and SoHO). The paper by Luhmann et al. (2007) discusses some of the questions that can be addressed by new perspectives on solar eruptions. In this paper we focus on objectives that can be addressed with data from the LET. 
Fig. 3 Photo of the LET and HET and the SEP Central housing. LET is mounted on a post so as to clear the FOV. HET is mounted on top of the SEP Central housing, with its electronics mounted inside. The SEP Central processor and the low-voltage power supply and bias supply for the four SEP sensors are located in this housing

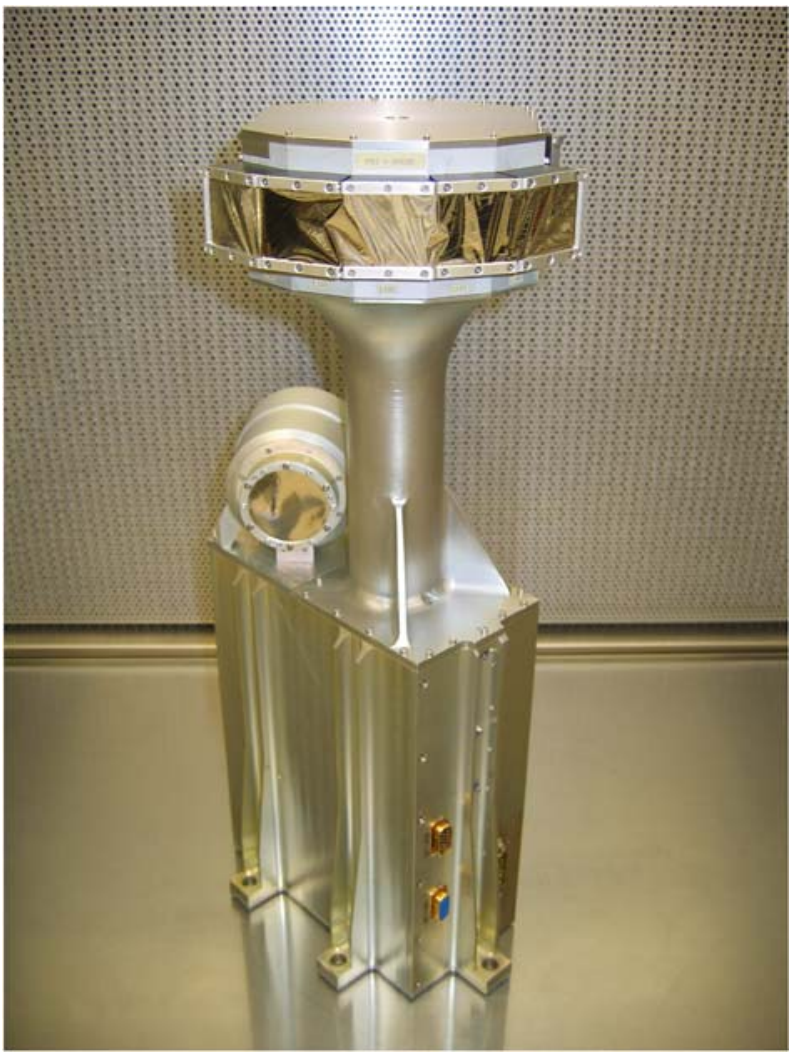

\subsection{SEP Acceleration by CME-Driven Shocks}

By 1995 it was well accepted that solar energetic particle (SEP) events generally fell into two distinct categories: "impulsive" and "gradual" (Reames 1995). According to this description, impulsive events are generated by acceleration in solar flares and are characterized by particle intensities generally lasting for hours and confined to narrow ranges of solar longitude, enhanced intensities of ${ }^{3} \mathrm{He}$ relative to ${ }^{4} \mathrm{He}$ and $\mathrm{Fe}$ relative to $\mathrm{O}$, and enhanced abundances of high charge state ions (e.g., $\mathrm{Fe}^{20+}$ ) relative to the solar wind. With some notable exceptions, these events are not generally associated with coronal mass ejections (CMEs). In contrast, gradual events are larger in peak intensity, last for days, and have a composition that is variable but generally more similar to the solar wind in elemental abundances and average charge states. Gradual events are a result of shock acceleration by CME-driven shocks in the corona and solar wind.

With the launch of ACE and the subsequent availability of detailed compositional measurements in many SEP events, this simple categorization of SEP events was called into question (e.g., Cohen et al. 1999; Mason et al. 1999; Mewaldt 2000; Mewaldt et al. 2006). Many large events have been observed that have elemental composition similar to that of impulsive events (Fig. 6), while having solar associations (CMEs, long X-ray flare duration) expected of gradual events. The average charge state of $>20 \mathrm{MeV} /$ nucleon heavy ions was also measured by SAMPEX, and, in many gradual events, was found to be similar to those of impulsive events (Labrador et al., 2003, 2005). Given the similarity in composition be- 
Table 1 Summary of LET characteristics

\begin{tabular}{|c|c|c|}
\hline Characteristic & Value & Details \\
\hline Measurement objective & $\begin{array}{l}\text { Composition, energy spectra, time } \\
\text { variations of solar and interplanetary } \\
\text { energetic particles }\end{array}$ & Sects. $2.1-2.5$; Figs. $4-18$ \\
\hline Measurement technique & $\begin{array}{l}\text { Multiple- } \Delta E \text { vs. residual energy, with } \\
\text { corrections for trajectory }\end{array}$ & Sects. 3.1, 3.3; Figs. 20-22 \\
\hline $\begin{array}{l}\text { Sensor system } \\
\text { Energy-loss } \\
\text { measurements }\end{array}$ & $\begin{array}{l}\text { One double-ended detector stack composed } \\
\text { of } 14 \text { ion-implanted Si detectors of varying } \\
\text { thickness and segmentation }\end{array}$ & $\begin{array}{l}\text { Sects. } 3.3,3.4 \text {, } \\
\text { Appendix 1; Figs. 19-20 }\end{array}$ \\
\hline $\begin{array}{l}\text { Trajectory } \\
\text { measurements }\end{array}$ & $\begin{array}{l}\text { Five segmented apertures on opposite sides } \\
\text { arranged in a fan and backed up by a } 1-\mathrm{D} \\
\text { position-sensitive detector with } 10 \text { segments }\end{array}$ & $\begin{array}{l}\text { Sects. } 3.3,3.4,4.7 \\
\text { Figs. } 20,23,38\end{array}$ \\
\hline $\begin{array}{l}\text { Onboard particle } \\
\text { identification }\end{array}$ & $\begin{array}{l}\text { Sixteen species from } \mathrm{H} \text { to Fe identified } \\
\text { within an average of } 12 \text { energy intervals }\end{array}$ & $\begin{array}{l}\text { Sects. 3.6.4, Appendixes 2, } \\
\text { 3, 4; Figs. 29-31, 41, } 42\end{array}$ \\
\hline Charge interval & & Sects. 3.6.4, 4.6; Fig. 1 \\
\hline Primary interval & $1 \leq Z \leq 28$ & \\
\hline Extended interval & $1 \leq Z \leq 83$ & \\
\hline Energy interval & & Sects. 3.6.4, 4.5, 4.6; \\
\hline $\mathrm{H}, \mathrm{He}$ & $1.8-15 \mathrm{MeV} /$ nucleon & Figs. 1, 29, 31, 32, 37 \\
\hline $\mathrm{O}$ & 3.4-33 MeV/nucleon & \\
\hline $\mathrm{Si}$ & 4.0-45 MeV/nucleon & \\
\hline $\mathrm{Fe}$ & 3.8-59 MeV/nucleon & \\
\hline Field of view & Two $133^{\circ}$ by $29^{\circ}$ fans & Sects. 3.3, 4.7; Figs. 20, 38 \\
\hline Geometry factor & $4.0 \mathrm{~cm}^{2} \mathrm{sr}$ & $\begin{array}{l}\text { Sects. 4.5-4.6; } \\
\text { Table 13; Fig. } 37\end{array}$ \\
\hline $\begin{array}{l}\text { Event yields } \\
\text { (large SEP event) }\end{array}$ & $>10^{6} \mathrm{H} \& \mathrm{He} ;>10^{5} \mathrm{C}-\mathrm{Si} ;>10^{4} \mathrm{~S}-\mathrm{Fe}$ & Sect. 4.8; Fig. 39 \\
\hline Element resolution & $<0.2$ charge units & $\begin{array}{l}\text { Sects. } 4.2,4.5 \text {; } \\
\text { Figs. } 22,35\end{array}$ \\
\hline Mass resolution & $\begin{array}{l}\text { Range } 2 \text { He: } 0.23 \mathrm{amu} \\
\text { Range } 3 \text { He: } 0.20 \mathrm{amu}\end{array}$ & Sect. 4.5 \\
\hline \multicolumn{3}{|l|}{ Resource allocations } \\
\hline Dimensions $(1 \times \mathrm{w} \times \mathrm{h})$ & $16.8 \mathrm{~cm} \times 14.8 \mathrm{~cm} \times 5.8 \mathrm{~cm}$ & Fig. 19 \\
\hline Mass & $0.855 \mathrm{~kg}$ & Sect. 3.10; Table 12 \\
\hline Instrument power & $1.18 \mathrm{~W}$ (excludes power supply ineffic.) & Sect. 3.10; Table 12 \\
\hline Bit rate & $577 \mathrm{bps}$ & $\begin{array}{l}\text { Sect. } 3.10 ; \\
\text { Tables } 12,16\end{array}$ \\
\hline
\end{tabular}


Fig. 4 Daily intensity of 7-90 $\mathrm{MeV} /$ nucleon oxygen over the period from September 1997 through October 2006. The smoothly varying background is due to anomalous cosmic ray and galactic cosmic ray oxygen, while the spikes are due to large SEP events. The data are from the SIS instrument on ACE

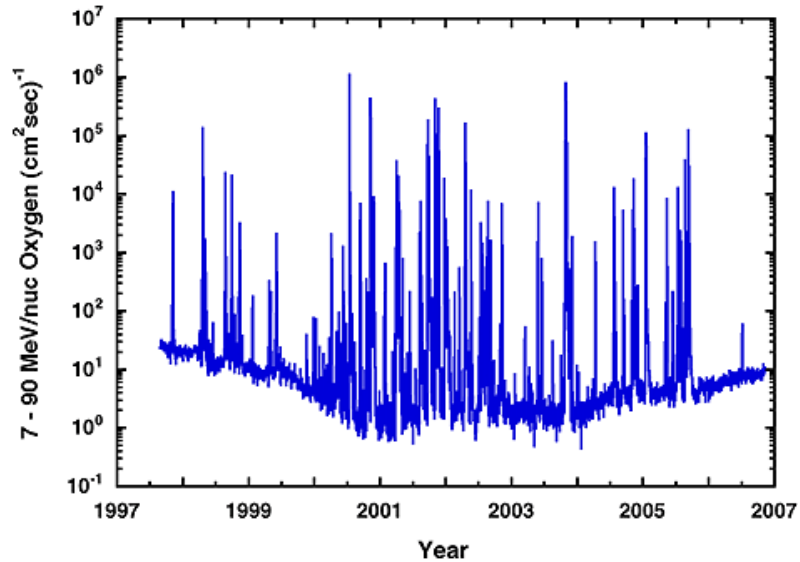

Fig. 5 Quiet-time ACR and GCR spectra for oxygen are shown along with the average intensity of oxygen during the first 34 hours of the January 20, 2005 SEP event. The SEP data are from the SIS and ULEIS instruments on ACE (Mewaldt et al. 2005a). The GCR and ACR data (Leske et al. 2000) are from the CRIS (blue triangles) and SIS (black circles) instruments on ACE and the LEMT instrument (green triangles) on Wind. Note that the ACR spectra are present only during solar minimum while large SEP events occur mainly during solar maximum

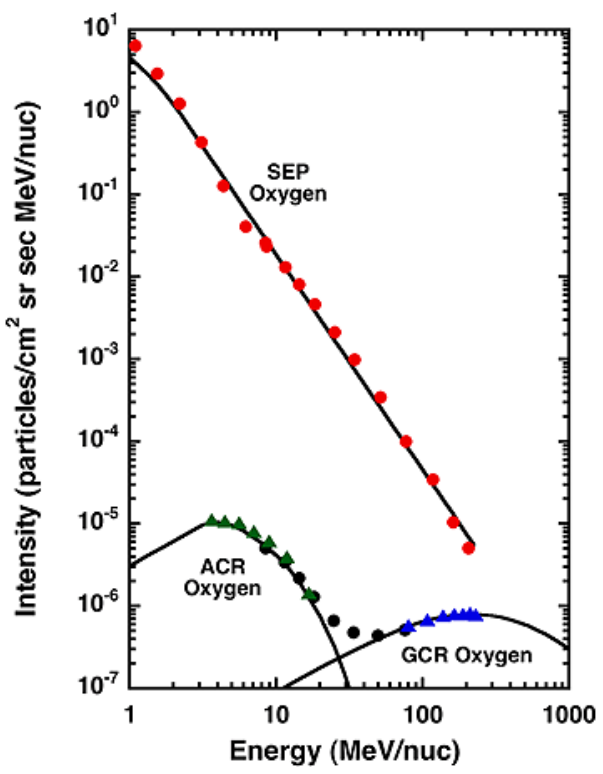

tween these "hybrid" events and impulsive events (thought to be accelerated flare material), most proposed explanations for the generation of hybrid events involve mixing flare material with ambient coronal or solar wind material. Table 2 summarizes the characteristics of SEP events according to the two-class picture, and also lists some of the new results from solar cycle 23 that have modified this picture.

The hypothesis by Tylka et al. (2005) suggests that the suprathermal population from which SEP events are accelerated has an energy-dependent composition, moving from more solar-wind-like at lower energies to more flare-like at higher energies (Fig. 7). Under the assumption that perpendicular shocks have a higher injection threshold than parallel shocks, acceleration at perpendicular shocks would result in more flare-like material being accelerated to produce a SEP event with enhanced $\mathrm{Fe} / \mathrm{O},{ }^{3} \mathrm{He} /{ }^{4} \mathrm{He}$, and elevated heavy ion charge states. In contrast, parallel shocks would predominantly accelerate suprathermal material with "nominal" composition resulting in typical gradual SEP events. A competing hypoth- 
Fig. 6 Composition of four SEP events observed by ACE/SIS as compared to standard abundances for gradual events (open circles) and impulsive events (filled circles). Cohen et al. (1999) found that these and other large SEP events associated with CMEs appeared to have an impulsive composition, including enhancements of ${ }^{3} \mathrm{He}$
Nominal=|mpulsive $O$ Nominal=Gradual

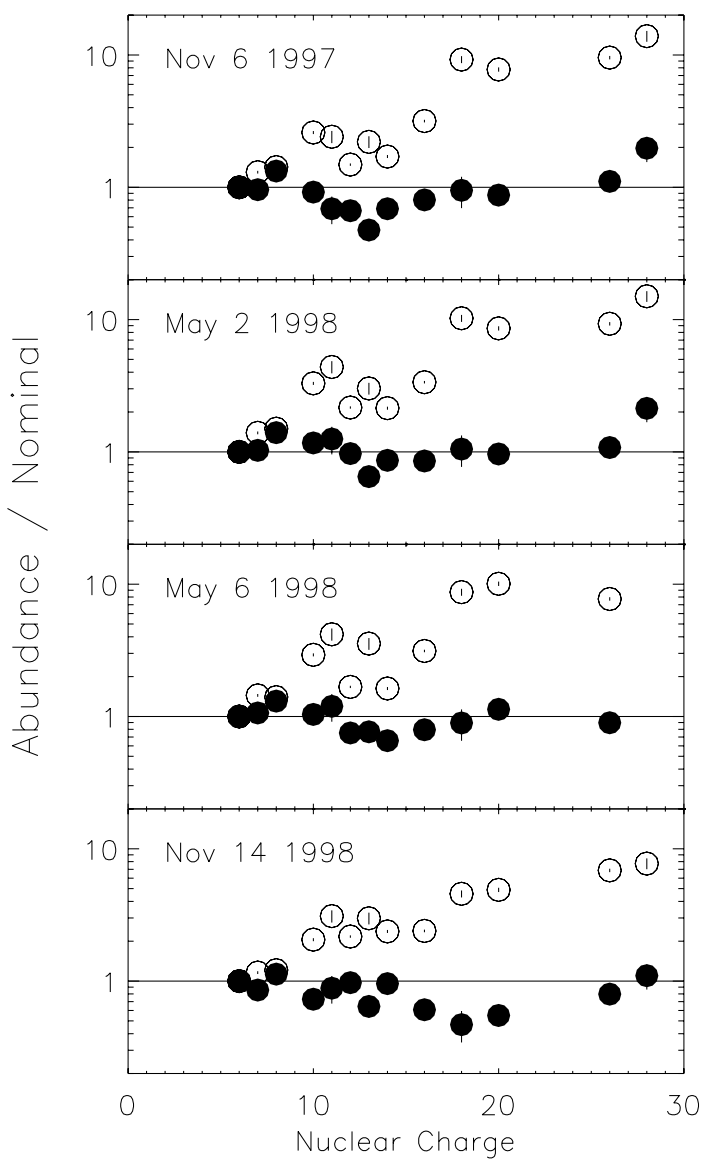

esis by Cane et al. $(2003,2006)$ involves a simpler scenario of flare-accelerated material superimposed on shock-accelerated material to produce a composite SEP event with an energy-dependent composition. The relative intensity of the two components depends on the strength of the shock, the size of the flare and flaring region, and the magnetic connection to the observer.

Energy-dependent composition has also been observed in events that are clearly dominated by shock-accelerated material. The inability of the shock to confine particles to the acceleration region results in "spectral breaks" such as those apparent in Fig. 8. As the turbulence near the shock is the dominant mechanism for returning particles to the shock, one would expect the relative energies of the break points for different ions to be a function of the particles' mean free paths or rigidity. This was found to be a reasonable approximation for the large SEP events of October/November 2003 (Cohen et al. 2005; Mewaldt et al. 2005b, 2005c), as well as others (Cohen et al. 2007). Modeling of shocks shows that the strength of the shock and its orientation varies along the face of the shock (Fig. 9).

There are several ways in which data from LET can further the understanding of CMEdriven shock acceleration and the generation of hybrid events. For the first time since the Helios spacecraft (Schwenn and Marsch 1991), SEP events will be measured simultane- 
Table 2 Characteristics of SEP events

\begin{tabular}{|c|c|c|c|c|}
\hline \multirow[t]{2}{*}{ Characteristic } & \multicolumn{2}{|c|}{$\begin{array}{l}\text { Conventional picture } \\
\text { (Reames 1999) }\end{array}$} & \multicolumn{2}{|l|}{ New results } \\
\hline & Impulsive & Gradual & Impulsive & Gradual \\
\hline${ }^{3} \mathrm{He} /{ }^{4} \mathrm{He}$ & $\sim 1$ & $\sim 0.0004$ & $>0.01$ (a) & $\sim 0.001-0.1(\mathrm{~b})$ \\
\hline $\mathrm{Fe} / \mathrm{O}$ & $\sim 1$ & $\sim 0.1$ & & $\begin{array}{l}\sim 0.01-1 \\
\text { often energy } \\
\text { dependent }(\mathrm{c})\end{array}$ \\
\hline $\mathrm{H} / \mathrm{He}$ & $\sim 10$ & $\sim 100$ & & \\
\hline Fe Q-State & $\sim 20$ & $\sim 14$ & $\begin{array}{l}\sim 13-20 \\
\text { broad distribution, } \\
\text { energy depend (d) }\end{array}$ & $\begin{array}{l}\sim 10-20 \\
\text { can be energy } \\
\text { dependent }(\mathrm{e})\end{array}$ \\
\hline Electron/Proton & Electron-rich & Proton-rich & & \\
\hline $\mathrm{X}$-rays & Impulsive & Gradual & & \\
\hline Typical duration & Hours & Days & & \\
\hline $\begin{array}{l}\text { Longitudinal } \\
\text { cone }\end{array}$ & $<30^{\circ}$ & $\sim 180^{\circ}$ & & \\
\hline Radio type & III, V (II) & II, IV & & II, IV, III- $\ell$ (f) \\
\hline Coronagraph & - & CME $96 \%$ & $\begin{array}{l}\text { Frequently CMEs, } \\
\text { often narrow }(\mathrm{g})\end{array}$ & $\begin{array}{l}\text { Always CME, usually } \\
\text { wide/fast (h) }\end{array}$ \\
\hline $\begin{array}{l}\text { Enhancements of } \\
(Z \geq 33) / O\end{array}$ & & & up to $10^{4}$ (i) & $0.2-20(\mathrm{j})$ \\
\hline $\begin{array}{l}\text { Heavy isotope } \\
\text { enhancements }\end{array}$ & & & $\begin{array}{l}\text { up to factors } \\
\text { of } \sim 5(\mathrm{k})\end{array}$ & $\begin{array}{l}\text { up to factors } \\
\text { of } \sim 3(1)\end{array}$ \\
\hline
\end{tabular}

(a) Ho et al. (2005)

(b) Desai et al. (2006)

(c) Cohen et al. (1999), Cane et al. (2006), Desai et al. (2006), Cohen et al. (2005), Mewaldt et al. (2006)

(d) Klecker et al. (2006)

(e) Labrador et al. (2003), Mazur et al. (1999)

(f) Cane et al. (2002)

(g) Wang et al. (2006a)

(h) Kahler and Vourlidas (2005)

(i) Reames and Ng (2004), Mason et al. (2004), Leske et al. (2007a)

(j) Reames and Ng (2004)

(k) Wiedenbeck et al. (2003), Leske et al. (2003b)

(1) Leske et al. (2007b)

ously at different solar longitudes. In combination with other SEP instruments (e.g., HET and SIT) on STEREO, particle spectra for many heavy ions (H through Fe) will be measured over a large energy range ( $\sim 0.04$ to $\sim 100 \mathrm{MeV} /$ nucleon $)$, something that was not possible with Helios. Such measurements are critical for understanding compositional variations in SEP events. The ability to make multipoint determinations of heavy-ion spectra over a large energy range is a crucial step in differentiating between current theories-the direct flare hypothesis of Cane et al. (2003, 2006), which predicts a longitudinal dependence 
Fig. 7 Schematic illustration of the model of Tylka et al. (2005). The higher injection threshold at quasi-perpendicular shocks results in the acceleration of mainly suprathermal ions that are enriched in remnant flare material. At quasi-parallel shocks the injection threshold is assumed to be lower, and a more normal composition is accelerated

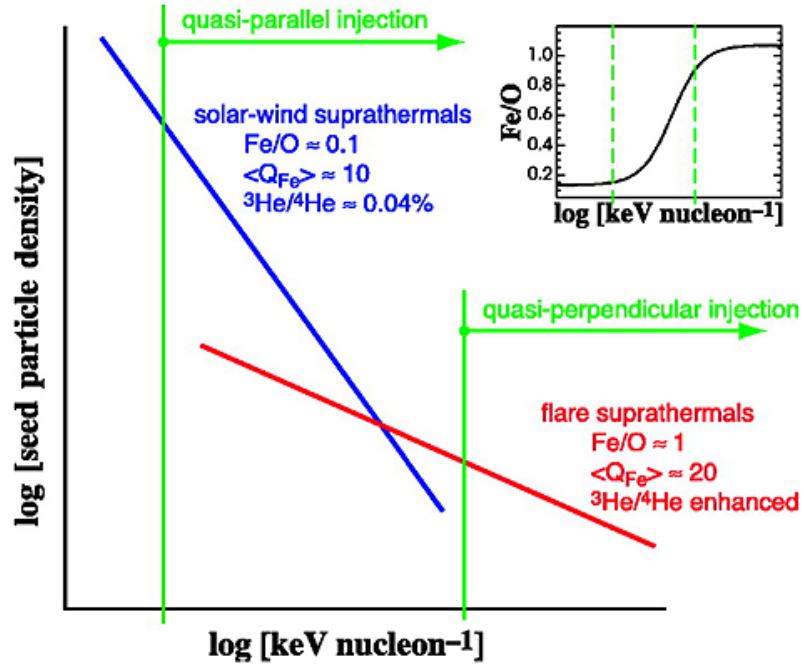

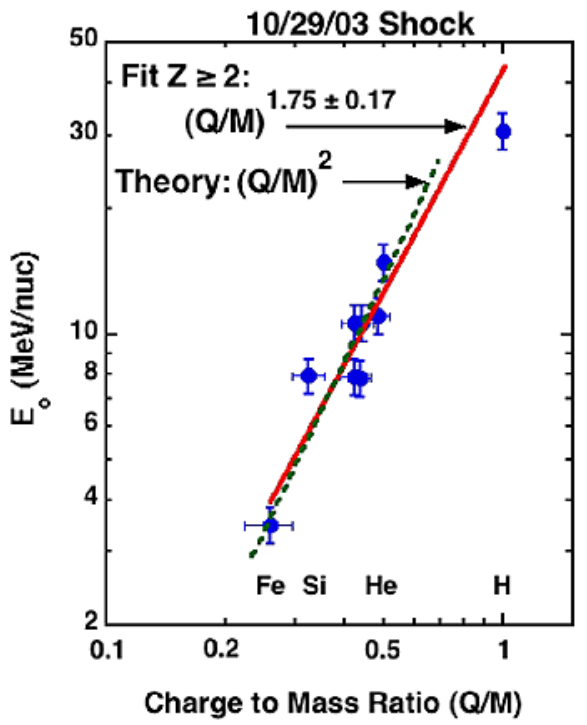

Fig. 8 Example of spectral breaks observed in the October 28, 2003 SEP event following the arrival of the shock at ACE (from Mewaldt et al. 2005c). In the left panel spectra measured by ACE and GOES are fit with a power-law times an exponential form with e-folding energy $E_{0}$. In the right panel the $E_{0}$ values are plotted vs. the charge-to-mass ratio $(Q / M)$ measured by SAMPEX (Labrador et al. 2005). The observed slope of $1.75 \pm 0.17$ is reasonably close to that predicted by the model of $\mathrm{Li}$ et al. (2005)

in the presence of flare-like material, and the shock-orientation model of Tylka et al. (2005), which does not, on average, predict a longitudinal dependence. Although the current SEP instrumentation boasts measurements made over a larger energy range with greater sensitivity 


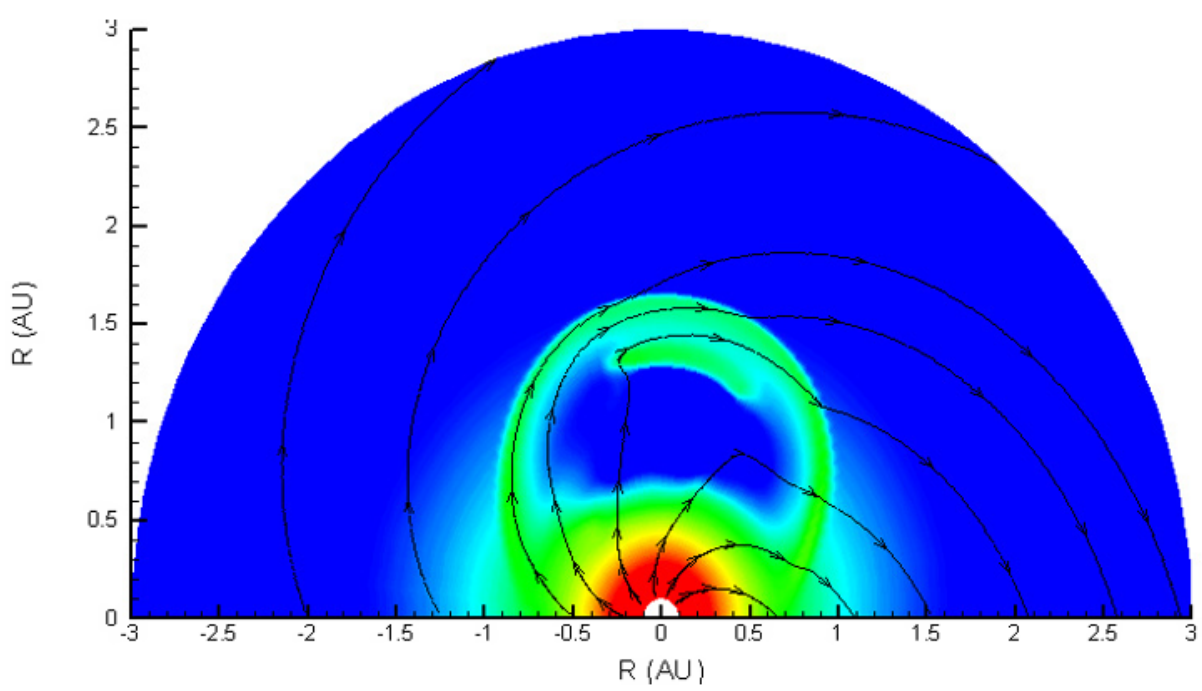

Fig. 9 Results of a shock simulation (see, e.g., Rice et al. 2003) shows density enhancements (color) around an expanding CME. The increases in density indicate the shock region and strength. The orientation of the magnetic field lines (black arrows) changes in relation to the shock normal along the flanks and nose of the shock

Fig. 10 Longitude distribution of $\mathrm{Fe} / \mathrm{O}$ ratios (divided by 0.134 ) measured in SEP events (from Cane et al. 2006). The predominance of Fe-rich events in the western hemisphere has been interpreted as evidence for direct contributions of flare accelerated particles in some well-connected events

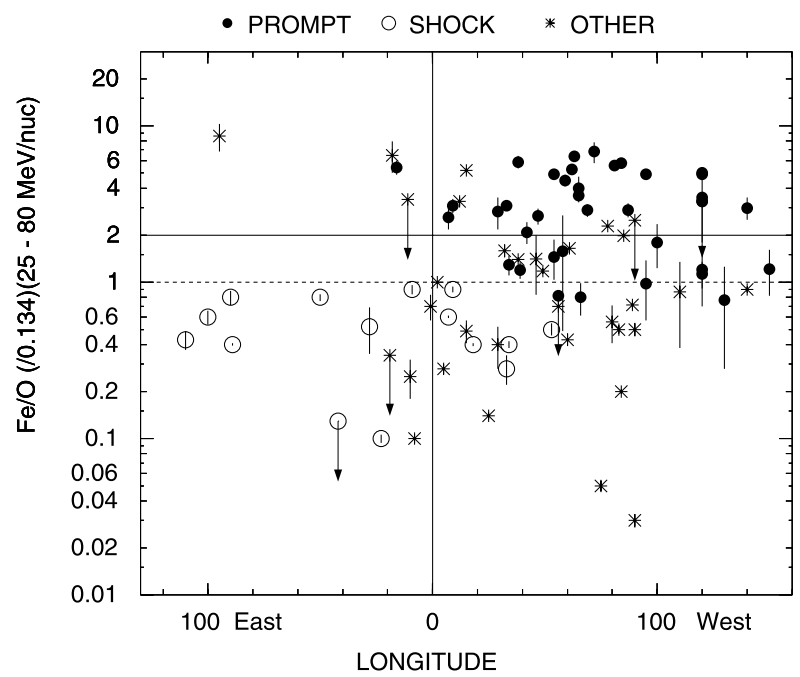

than previously possible (Stone et al. 1998a), longitudinal studies using such instrumentation (e.g., von Rosenvinge et al. 2001) have not made significant progress over similar research performed decades ago (e.g., Mason et al. 1984) largely because they have been statistical in nature (Fig. 10) and have not involved multipoint measurements. With STEREO other aspects of SEP events, such as the energy of spectral breaks, the spectral index at high and low energies, and the temporal evolution of spectra, intensities, and composition can be examined as a function of longitude within a single SEP event, providing key information for testing models of SEP acceleration and transport. 


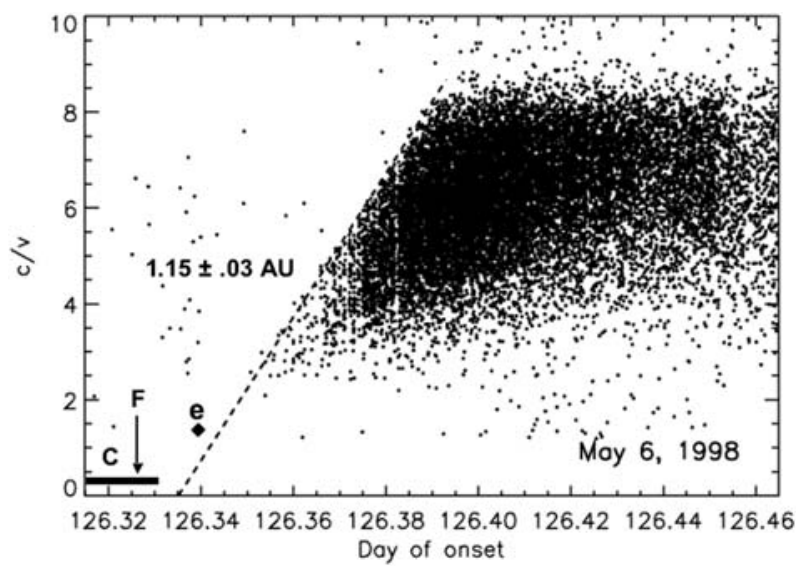

Fig. 11 The measured value of $c / v$ (where $c$ is the speed of light and $v$ is the particle velocity) is plotted vs. arrival time of individual $\mathrm{C}$ to $\mathrm{Ni}$ ions with $>7 \mathrm{MeV} /$ nucleon observed by ACE/SIS during the May 6, 1998, SEP event (Mewaldt et al. 2003). Also shown is the onset time for near-relativistic electrons (Haggerty and Roelof 2001), the onset of the X-ray flare, and the CME release time (band includes range of two extrapolation approaches). A fit to the onset gives an interplanetary pathlength of $1.15 \pm 0.03 \mathrm{AU}$ and a particle release time near the Sun of 8:02 \pm 2 minutes

The source of the energetic particles can also be examined through timing studies. Using the arrival time and energy of energetic particles, the particle release time near the Sun can be deduced (Fig. 11). This time can then be compared to the height vs. time profiles of CMEs, temporal evolution of flares, and the timing of other solar phenomena. Such studies have been performed with events observed by instruments on ACE, Wind, and SoHO (e.g., Krucker and Lin 2000; Haggerty and Roelof 2002; Mewaldt et al. 2003; Tylka et al. 2003; Klassen et al. 2005) in an effort to determine the likely accelerator (CME-driven shock or flare-related reconnection) of the particles. Although there is statistical evidence for a dependence of SEP onset timing on connection longitude, it is not known if this is due to the time it takes for the shock to reach the field line on which the spacecraft is located, or the time it takes for particles to diffuse to this field line. Multipoint timing studies may be able to decide between these possibilities.

Finally, during SEP events the combination of data from STEREO and ACE (located at the inner Lagrange point) will allow the seed particle population to be studied along with the resulting accelerated population. This cannot be done by a single spacecraft as the seed and accelerated populations travel along different trajectories from the Sun (the seed population taking a more radial path, while the accelerated particles are generally flowing along the Parker spiral). However, with two or more separated spacecraft the composition of the seed and accelerated populations can be measured and compared (see Fig. 12).

\subsubsection{Comparison of SEP and CME Kinetic Energies}

The particles accelerated by CME-driven shocks derive their energy from the kinetic energy of the CME. It is of interest to compare the total kinetic energy of SEPs in large SEP events to the kinetic energy of the CME measured by coronagraphs in order to evaluate the efficiency of SEP acceleration. This efficiency is known to vary over many orders of magnitude (see, e.g., Kahler and Vourlidas 2005). Figure 13 shows a comparison of the kinetic energy of SEPs vs. the CME kinetic energy for 17 large SEP events from solar cycle 23 (Mewaldt 


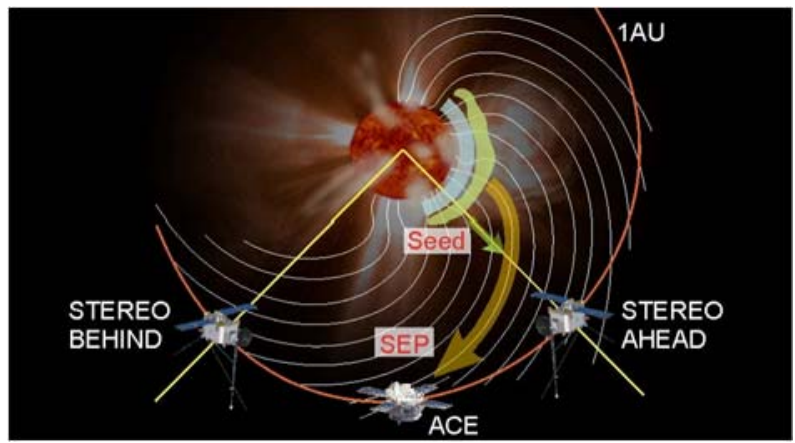

Fig. 12 Schematic of the trajectories of the accelerated and seed particle populations and their relation to the positioning of the STEREO and ACE spacecraft. For CME-driven shocks originating in the western hemisphere of the Sun, the Ahead STEREO spacecraft will be able to measure the seed populations for the accelerated SEPs, which follow the magnetic field lines and will be measured by either ACE or Behind (typically $\sim 50^{\circ}$ to the west of where they were accelerated, depending on the solar wind velocity). Thermal plasma travels radially, while suprathermal particles will be distributed in longitude when they reach 1 AU, depending on their velocity and on how much they scatter in the inner heliosphere. In the above picture the Behind STEREO spacecraft will also be situated to image the CME as is moves away from the Sun

Fig. 13 Comparison of CME and SEP kinetic energies for 17 large SEP events (from Mewaldt et al. 2005d). The single square is the April 21, 2002 event; the circles are the October-

November 2003 events, and the diamonds are other events where CME mass estimates were available

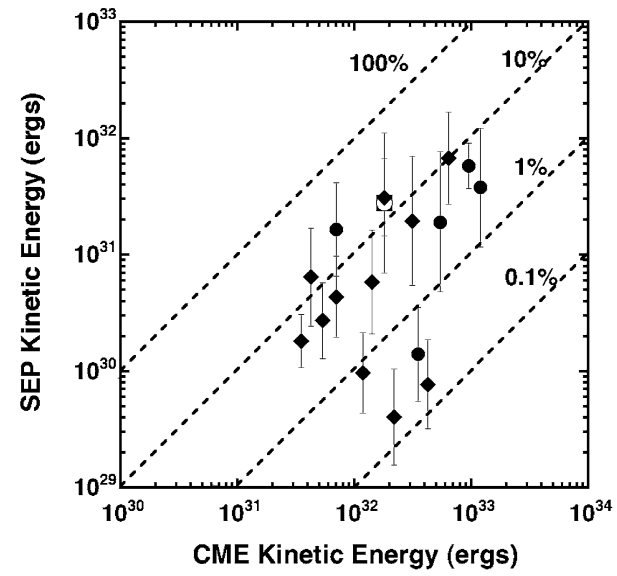

et al. 2005d; Mewaldt 2006). The SEP kinetic energies were obtained by integrating the measured and extrapolated SEP spectra from 0.01 to $100 \mathrm{MeV} /$ nucleon. Note that while there is a wide range of deduced efficiencies, it is not uncommon for the SEP kinetic energy to amount to $\sim 10 \%$ of the CME kinetic energy. It is interesting that a similar efficiency is required for the acceleration of galactic cosmic rays by supernova shock waves to sustain the cosmic ray intensity in the Galaxy (Ptuskin 2001).

The data in Fig. 13 are from single-point measurements of SEPs with near-Earth instruments that were corrected for the longitude of the associated flare and CME. This correction is uncertain, and undoubtedly depends on the configuration of the interplanetary magnetic field. With measurements of SEP spectra over a wide energy range from the two STEREO spacecraft and from ACE and GOES near Earth it will be possible to measure directly the longitudinal variation in SEP intensities for many SEP events, thereby increasing the ac- 
curacy of the SEP energy estimates. The best estimates of CME kinetic energies are for limb CMEs, while estimates for halo-CMEs are more uncertain (Vourlidas et al. 2000). With STEREO there should be CME images of the events from two or three points of view, thereby providing much more accurate estimates of the CME kinetic energy.

\subsection{Impulsive Solar Energetic Particle Events}

As noted earlier there exists a second category of solar energetic particle events. These are frequently called "impulsive" events because they tend to occur in association with impulsive solar X-ray events (i.e., events with fast onsets and short durations). A variety of observational characteristics distinguishing impulsive from gradual events have been suggested (see Table 2 and Sect. 2.1), many of which rely on composition signatures. The most distinctive indicator of an impulsive event is a large enhancement of the ${ }^{3} \mathrm{He} /{ }^{4} \mathrm{He}$ ratio, which often can be $>1,000 \times$ the solar wind value of $\sim 4 \times 10^{-4}$. Thus the term "3 He-rich" is often used as a synonym for impulsive SEP events.

Although it is widely believed that particle acceleration in impulsive events is driven by magnetic reconnection in solar flares, the detailed physical mechanisms are only poorly understood. A variety of resonant processes for heating the background plasma or otherwise extracting the population of particles to be accelerated from it have been proposed. To date, however, none of them has proven capable of accounting for all of the observed characteristics.

Measurements of impulsive SEP events over a relatively broad energy range (Mason et al. 2000, 2002) show that spectral shapes can differ from event to event and among different species in an individual event. Thus, it is not sufficient to characterize impulsive events by their composition derived from observations made over a narrow range of energies. The spectral variability may be responsible for the fact that correlations among various abundance ratios are often relatively weak (Reames et al. 1994). On STEREO the broad energy coverage provided by the combination of SIT, SEPT, LET, and HET will provide the spectral measurements needed to better understand the observed composition. Figure 14 compares the energy coverage and sensitivities of SIT and LET with spectra of key species that have been reported in two impulsive events. The overlap in energy coverage between the various SEP instruments will also help facilitate their intercalibration using particle measurements made in flight.

The heavy-element $(Z \geq 6)$ composition observed in most impulsive SEP events follows a pattern of enhancements (relative to solar wind or to gradual SEP composition) that increase approximately monotonically with atomic number (or mass). However, a small number of events have been reported with large deviations from this pattern (Mason et al. 2002; Wiedenbeck et al. 2003). In addition, isotopic composition measurements have shown that impulsive events frequently have strongly enhanced abundances of heavy isotopes (Mason et al. 1994; Dwyer et al. 2001; Wiedenbeck et al. 2003). Observed values of the ${ }^{22} \mathrm{Ne} /{ }^{20} \mathrm{Ne}$ ratio $>3 \times$ the solar wind value are larger than seen in any but the most extremely fractionated gradual events (Leske et al. 2003a), as illustrated in Fig. 15. Thus far there have been relatively few well-measured examples of impulsive SEP events with extreme elemental and isotopic fractionation patterns. STEREO will search for additional events of this sort, measure their composition over a broad energy range, and provide context information that should help in identifying the features that distinguish these highly fractionated events from the general population of impulsive SEP events.

Recent measurements from Wind/LEMT (Reames and Ng 2004), ACE/ULEIS (Mason et al. 2004), and ACE/SIS (Leske et al. 2007a) show that some impulsive SEP events exhibit 
Fig. 14 The average ${ }^{3} \mathrm{He}$ and

${ }^{4} \mathrm{He}$ intensities for two ${ }^{3} \mathrm{He}$-rich SEP events measured by the ULEIS and SIS instruments on ACE are plotted vs.

energy/nucleon. The sensitivity of the SIT and LET instruments for measuring energy spectra of ${ }^{3} \mathrm{He}$ and ${ }^{4} \mathrm{He}$ is also indicated (solid line) as the intensity at which one event per 3 hrs would be collected in a given energy bin [using the energy bins in Fig. 30 for LET and in Mason et al. (2007) for SIT]
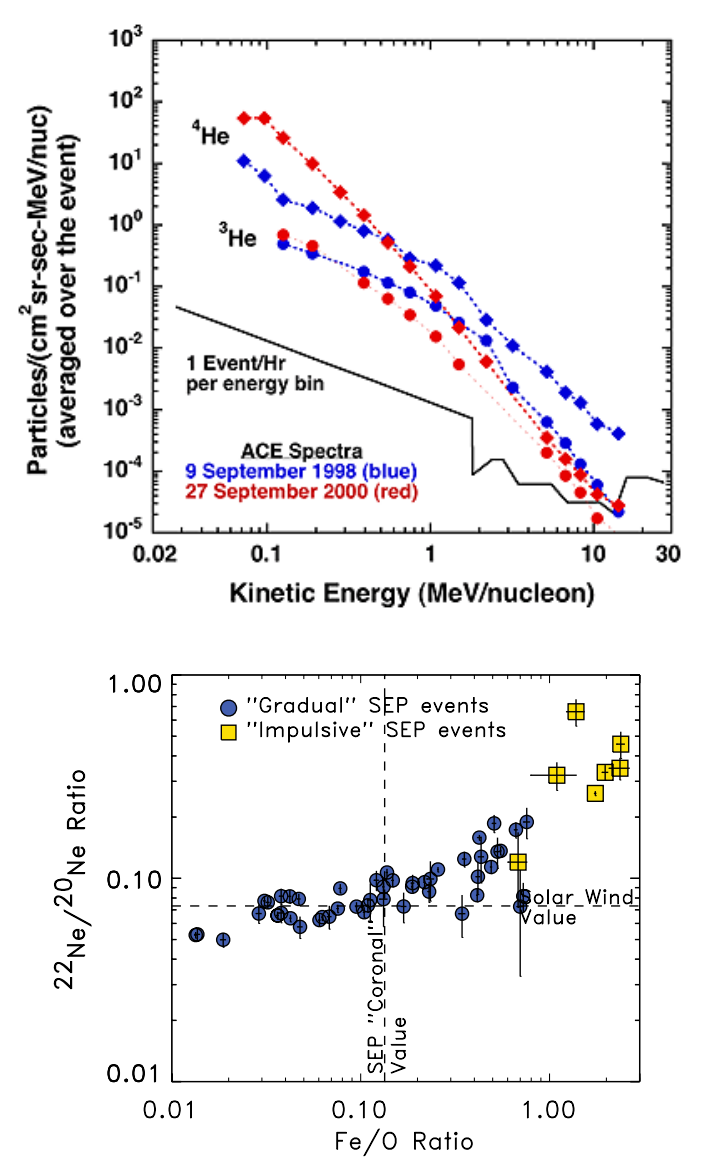

Fig. 15 Plot of the ${ }^{22} \mathrm{Ne} /{ }^{20} \mathrm{Ne}$ isotope ratio vs. $\mathrm{Fe} / \mathrm{O}$ for a number of SEP events observed with the ACE/SIS instrument from 1997 through 2005. The impulsive events shown, which are indicated by yellow squares, are among the largest ${ }^{3} \mathrm{He}$-rich events observed by SIS. Note that both elemental and isotopic fractionation tends, on average, to be more extreme in impulsive than in gradual events

large abundance enhancements of ultraheavy ( $\mathrm{UH}, Z \geq 30$ ) elements relative to standard solar composition that tend to increase with increasing $Z$. In some cases these enhancements can be $>10^{2}-10^{3}$, comparable to observed ${ }^{3} \mathrm{He}$ enhancements. These observations provide new constraints on theories of particle acceleration in impulsive SEP events since the UH species occur with a wide range of $M / Q$ ratios and with such low abundances in the background plasma that they behave strictly as test particles. The studies from Wind/LEMT and ACE/ULEIS only resolved major charge groups, while the higher-resolution ACE/SIS data were restricted to elements with $Z \leq 40$ and had a relatively high energy threshold (>12 MeV/nucleon). The LET instrument, which has a significantly lower energy threshold than SIS and a dynamic range sufficient for measuring elements over the entire periodic table, should provide improved charge resolution for $Z>40$ in those impulsive events having significant intensities above several $\mathrm{MeV} /$ nucleon.

In a significant fraction of impulsive SEP events, particle distributions at $1 \mathrm{AU}$ are observed to be highly anisotropic and appear during their early phases as beams propagating outward from the Sun along the magnetic field. Such events tend to exhibit a dispersive onset, with the time of first arrival of particles of a given velocity, $v$, increasing linearly with $1 / v$. It is often (but not always) the case that the slope of this relation corresponds to the expected $\sim 1.2$ AU length of a nominal Parker spiral magnetic field line between the Sun and Earth as in Fig. 11. This behavior is taken as indicating nearly scatter-free propa- 
gation from the point of release at the Sun to $1 \mathrm{AU}$. Using the observed dispersion of onset times and/or the time evolution of the anisotropy as constraints, one can model the injection and propagation of SEPs in order to deconvolve the effects of injection and release times of finite duration from those of scattering during propagation (Wang et al. 2006b; Dröge et al. 2006). The LET instrument, with its capability for measuring arrival direction distributions within the ecliptic plane (see Sect. 4.7), will provide data for such studies. LET anisotropy observations will also help in the interpretation of data from the SIT and HET instruments (which make measurements within a limited field of view around the nominal magnetic field direction) when the particle fluxes have significant anisotropies. High velocity particles (e.g., $v / c>0.15$ ) are particularly useful for timing studies because they experience less propagation delay and provide more-precise time markers than very low-velocity particles. Although electrons generally yield the most precise timing data, the higherenergy ions measured by LET and HET will be important for understanding differences between the acceleration and release histories of ions and electrons (Krucker and Lin 2000; Mewaldt et al. 2003).

A major impediment to the study of impulsive SEP events has been the fact that such events tend to be associated with relatively small X-ray flares on the Sun. The particle data generally do not provide precise enough timing information to unambiguously distinguish which of many small X-ray events they are associated with. Following seminal work of Reames et al. (1985), considerable progress has recently been made in associating impulsive SEP events with their counterparts on the Sun (Wang et al. 2006a; Nitta et al. 2006). Timing associations between ion events and electron events observed in situ near 1 AU and then between the electron events and Type III radio bursts, which are triggered by the streaming of lower-energy electrons near the Sun, have made it possible, in some cases, to pinpoint the solar release time with sufficient accuracy to identify a unique solar-flare counterpart. This association can be checked using EUV or X-ray imaging data in conjunction with a model of the solar magnetic field: if the calculated field line from the imaged flare location through the corona does not correspond to an interplanetary field line that the Parker spiral will ultimately connect to the observer, the association is called into question.

For impulsive SEP events with clear solar flare associations one can then use sequences of EUV and white-light images to investigate dynamical changes in the solar corona associated with the particle release and obtain information about the physics that is involved. Such studies, which are still in their infancy, have provided intriguing evidence that impulsive SEP events originate when flaring loops undergo reconnection with adjacent open field lines and that the particle injections can be accompanied by escaping "jets" or narrow CMEs (Wang et al. 2006a). The STEREO mission will provide the key observations needed for making the sequence of connections essential to these studies: ion and electron measurements from the IMPACT/SEP instruments, radio burst data from SWAVES, EUV and white-light imaging from SECCHI. Measurements from RHESSI, GOES, Hinode, SoHO, ACE, and Wind as well as ground-based solar radio burst observatories will provide valuable supporting data.

Based on investigations that attempted to associate impulsive SEP events with specific solar flares on the Sun (Reames 1993) and multispacecraft studies involving Helios and ISEE-3 (Reames et al. 1991) it has been concluded that impulsive SEPs propagate into a $\sim 10^{\circ}-20^{\circ}$ half-angle cone about the magnetic field line connected to the source region. This narrow spread is attributed to the relatively small spatial region from which the particles are released, in contrast to the very broad region from which the shock-accelerated particles in gradual SEP events originate. However, as noted earlier, flare associations are difficult. It is not clear whether some associations with eastern hemisphere flares actually reflect a highly distorted magnetic connection between the Sun and the observer or some deficiency in our simple picture of impulsive SEP release and transport. 
It has been found that there is a maximum fluence of ${ }^{3} \mathrm{He}$ that can be obtained from an impulsive SEP event (Ho et al. 2005). This observation, which indicates that only a limited number of energetic ${ }^{3} \mathrm{He}$ particles can be released from the Sun in such an event, may also be related to the small spatial size of the acceleration region.

In the course of an impulsive SEP event the particle intensity seen at a single spacecraft can exhibit "dropouts" (Mazur et al. 2000). These have been explained in terms of the shredded character of the interplanetary field caused by the random walk of field lines (Giacalone et al. 2000). The related phenomenon of abrupt event onsets with no velocity dispersion is thought to be due to the observer moving into flux tubes already populated with particles.

The two STEREO spacecraft, together with ACE, Wind, and other spacecraft operating near Earth, will obtain measurements of individual impulsive SEP events from different heliolongitudes, thereby probing the longitudinal widths of these events at $1 \mathrm{AU}$ without having to rely on solar-flare associations. If impulsive SEPs do indeed have a narrow longitudinal spread, multispacecraft correlations will mainly be seen in the first one to two years of the STEREO mission. During this solar minimum period the interplanetary field should be relatively undisturbed, but impulsive SEP events may be rather infrequent. Investigation of correlations at larger angular separations and how they depend on solar activity could provide new insights into these events later in the mission. The plasma beta, which may play an important role in organizing the particle transport (Reames et al. 2001), will be available using data from the MAG and PLASTIC instruments.

\subsection{Space Weather}

\subsubsection{An Interplanetary SEP Network}

Although CME, X-ray, and radio observations all provide early warning of large solar eruptive events that are a space weather concern, the best way to accurately gauge how large a SEP event will be is with direct particle measurements from a location that is magnetically well connected to the particle acceleration site near the Sun, whether it be a CME-driven shock or a flare. This is illustrated in Fig. 16, which shows typical intensity profiles for SEP events originating at different longitudes. The two STEREO spacecraft ahead and behind Earth, combined with ACE (Stone et al. 1998a) and GOES (Onsager et al. 1996) near Earth, will form a three-point interplanetary network for early detection of large SEP events. It is likely that any large SEP event of concern in the nearEarth environment will be magnetically well connected to at least one of these spacecraft, all of which broadcast SEP intensities in real time. Algorithms now exist that forecast the eventual size of an event based on its onset profile (e.g., Hoff et al. 2003; Neal and Townsend 2005). With this network, alerts for large SEP events can be made sooner and with much greater precision. Real-time data from LET and HET will provide energy spectra of $\mathrm{H}, \mathrm{He}, \mathrm{CNO}$ and Fe from $\sim 3$ to $\sim 100 \mathrm{MeV} /$ nucleon, including the most important energy range for radiation effects on humans or hardware.

With the many spacecraft that are and will be exploring the inner heliosphere during the coming years, the growing set of assets that are exploring Mars, and the expected return of astronauts to the Moon, it is clear that there will be an increasing need for global models, forecasts, and "nowcasts" of space weather throughout the inner heliosphere, and not just along the Earth-Sun line. In particular, there will be a need to model the global longitude distribution of particle intensities from large SEP events as they occur (see, e.g., Turner 2006). SEP data from the three-point network described earlier will build up a statistical database of SEP longitude distributions, and it will provide valuable tests for global models that are driven by imaging and in situ observations. 

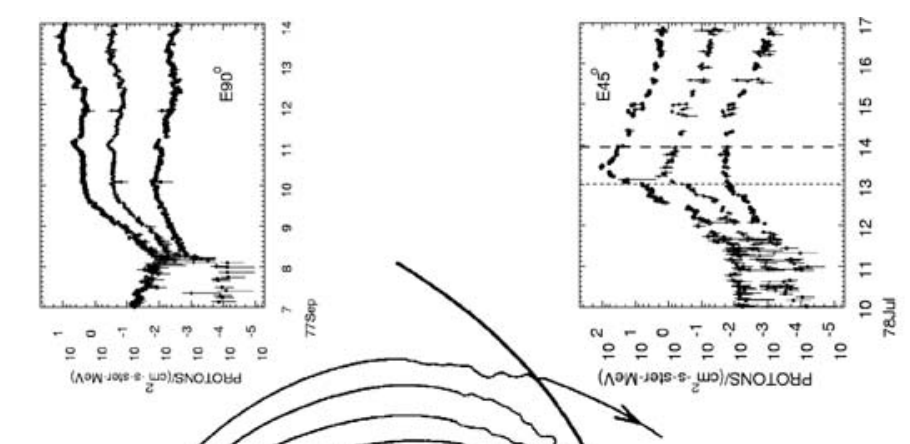

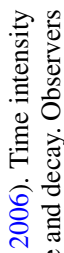

을

丞

ฮั ญ

可证

诃

눙

㵄.

홍

능

क

₹ี

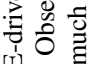

地

$\sum_{0} \sum_{0}$

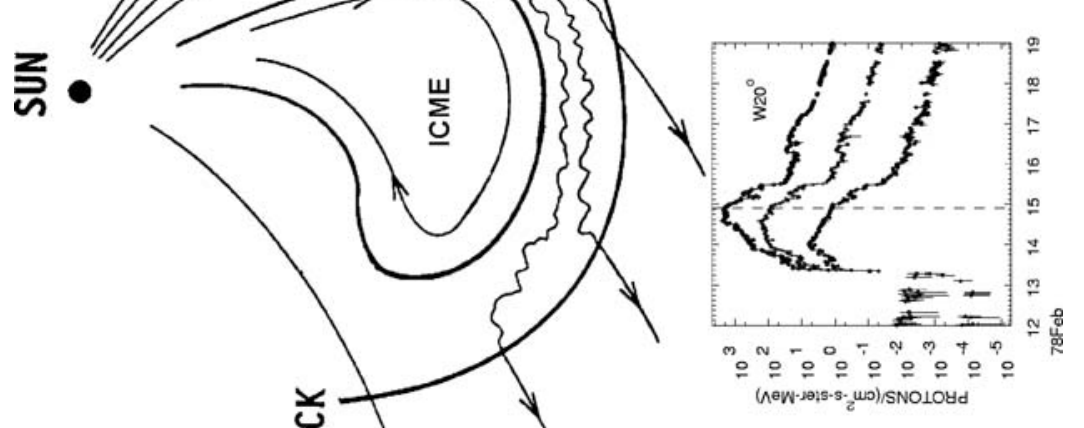

牙 咅

胥

吾过

कू क्षे

응효을

管

尊号言

巳 స్

흘

흔

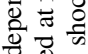

完

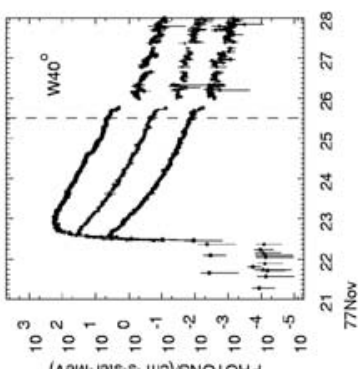

政

\% $\frac{3}{3}$

되욜

䆛

प०

os

등 궁

它

: .:

再爷

한

उ

突

记

ธ․

है

눙

当管。

ㄴ.

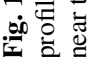


Interplanetary shocks are currently identified using both magnetic field and plasma instruments on ACE, providing 1-hour warning. However, to extend the warning time, the escaping upstream component of energetic storm particle (ESP, see Sect. 2.4) ion events can be used to provide short-term forecasts of approaching interplanetary shocks. ACE/EPAM data have been used to train an artificial neural network to predict the arrival time of the maximum intensity of an ESP event at Earth (Vandegriff et al. 2005). On STEREO, SIT, SEPT, and LET will all be sensitive to ions escaping upstream from approaching shocks, and it will be possible to have a three-point network to warn of interplanetary shocks.

\subsubsection{Characterizing the 1-AU Radiation Environment}

Knowledge of the long-term radiation environment is important for evaluating design requirements for spacecraft hardware. Although the intensity of GCRs in a given energy interval varies by at most a factor of 10 over the solar cycle, the intensity of SEPs with, e.g., 10 to $30 \mathrm{MeV} /$ nucleon can vary by a factor of $\sim 10^{6}$ (see Figs. 4 and 5). Indeed, ACE data have shown that the yearly average intensity of $>20 \mathrm{MeV} /$ nucleon $\mathrm{CNO}$ and Fe during the past solar maximum has been considerably greater than earlier in the space era, with three of the years from 2000 to 2003 exceeding the $90 \%$ confidence limits of Tylka et al. (1997) for yearly fluences of 21 to $43 \mathrm{MeV} /$ nucleon CNO and 45 to $95 \mathrm{MeV} /$ nucleon Fe (Mewaldt et al. 2007). These results from ACE indicate that models of the 1-AU radiation environment need to be updated. Data from LET and HET can complement ACE data in developing improved models of the radiation environment. In particular LET and HET provide $\mathrm{H}$ and He data in an interval where ACE has incomplete coverage, and where GOES data are often affected by relatively high background and by temporal variations in the geomagnetic cutoff at geosynchronous altitude.

\subsection{Acceleration by CIRs and ESP Events}

\subsubsection{Corotating Interaction Regions}

Corotating interaction regions (CIRs) occur when a stream of fast solar wind overtakes a stream of slow solar wind. The compression region that forms at the boundary can strengthen into a shock pair (forward and reverse shocks) and accelerate particles up to $\sim 20 \mathrm{MeV} /$ nucleon (see, e.g., Richardson 2004). As long as the two streams are consistently emerging from the Sun, the structure will corotate past fixed points as the Sun rotates, sometimes existing over many solar rotations.

The elemental composition of the accelerated particle population associated with CIRs is still a puzzle. Although some of the elemental ratios (e.g., Fe/O) are similar to those found in the solar wind, other ratios like $\mathrm{C} / \mathrm{O}$ are significantly enhanced (e.g., Mason et al. 1997). The cause of this remains unknown. Although it has been suggested that the CIR composition anomalies are due to preferential acceleration of seed particles arising from the "inner source" of pickup ions (Gloeckler 1999), measurements of the charge states of CIR-accelerated ions have not found evidence for singly charged C ions (Mazur et al. 2002; Möbius et al. 2002).

With the multispacecraft measurements from STEREO and ACE, it will be possible to examine the energy spectra and abundances of many heavy-ion species over a wide energy range simultaneously at three different spatial points in the CIR structure. The evolution of the spectra at these three points can also be measured as the structure corotates past the spacecraft. Under circumstances where both a forward and a reverse shock are formed by 
$1 \mathrm{AU}$, comparing the resulting accelerated particle population using the coincident measurements made in the leading and trailing regions of the CIR will yield valuable information concerning the characteristics and evolution of these two different shocks and the seed particles that are accelerated.

Typically CIR events exhibit inverse velocity dispersion (i.e., the lower energy ions arrive first) due to the fact that the shock strengthens as it moves outwards and so, initially, is only able to accelerate particles to lower energies (Reames et al. 1997). Later, when the shock is strong enough to generate higher energy particles, this dispersion effect is lessened as transport over longer distances affects the low energy ions more. Examining these signatures simultaneously at three different points in the large CIR structure will provide key information regarding the formation and evolution of the reverse shock.

\subsubsection{Energetic Storm Particles}

Similar to CIR events, energetic storm particle (ESP) events are a result of interplanetary shock acceleration which occurs relatively near the spacecraft (rather than primarily near the Sun, as in SEP events). ESP events provide one of the best opportunities to study shock acceleration of energetic particles since the characteristics of the particle population, as well as the characteristics of the shock, can be measured at the same time. With two well-separated spacecraft, STEREO will be able to examine the spatial extent and characteristics of traveling, interplanetary shocks and will also be able to observe composition differences resulting from differing seed populations. Comparing the measured ESP characteristics over a wide energy range with the magnetic field and plasma properties at several points will provide insight into the structure of the shock and related acceleration conditions.

\subsection{Anomalous Cosmic Rays}

Anomalous cosmic rays (ACRs) originate from both interstellar and heliospheric sources. The most abundant ACR species are those that have a large neutral component in the ISM, including $\mathrm{H}, \mathrm{He}, \mathrm{N}, \mathrm{O}, \mathrm{Ne}$, and $\mathrm{Ar}$ (see, e.g., Cummings et al. 2002). Interstellar neutral atoms of these elements drift into the heliosphere, are ionized by solar UV or charge exchange with the solar wind to become pickup-ions, and are then convected into the outer heliosphere where they are accelerated to energies of tens of MeV/nucleon (Fisk et al. 1974). Pesses et al. (1981) first proposed that ACRs are accelerated at the solar-wind termination shock by diffusive shock acceleration, and ACR energy spectra of these elements at $1 \mathrm{AU}$ and in the outer heliosphere out to $70 \mathrm{AU}$ are consistent with those expected from shock acceleration models (Cummings et al. 2002).

Although Voyager 1 did not observe the expected shock-accelerated power-law spectrum in unmodulated form when it crossed the termination shock (Stone et al. 2005; McDonald et al. 2005), Voyager 1 did find a weak shock with a compression ratio of $\sim 2.5$ (Burlaga et al. 2005). It is possible that ACRs are accelerated at another location on the termination shock (e.g., McComas and Schwadron 2006; Stone et al. 2005), and it has also been suggested that ACRs are accelerated in the heliosheath (e.g., Moraal et al. 2006; Zhang et al. 2006).

Figure 17 shows 1 AU spectra for N, O, Ne, and Ar that were measured by ACE and Wind during the 1997-1998 solar minimum period (Reames 1999; Leske et al. 2000). Also shown are five other species measured at $1 \mathrm{AU}$, four of which- $\mathrm{Mg}, \mathrm{C}, \mathrm{Si}$, and $\mathrm{S}-$ also exhibited low-energy increases at Voyager (as did Na) (Cummings et al. 2002), but of much lower intensity than the abundant ACR elements. Since species such as $\mathrm{Mg}, \mathrm{Si}$, and $\mathrm{Fe}$ should be either ionized or locked up in grains in the ISM (e.g., Slavin and Frisch 2002), their origin is unclear but must be from the heliosphere. 


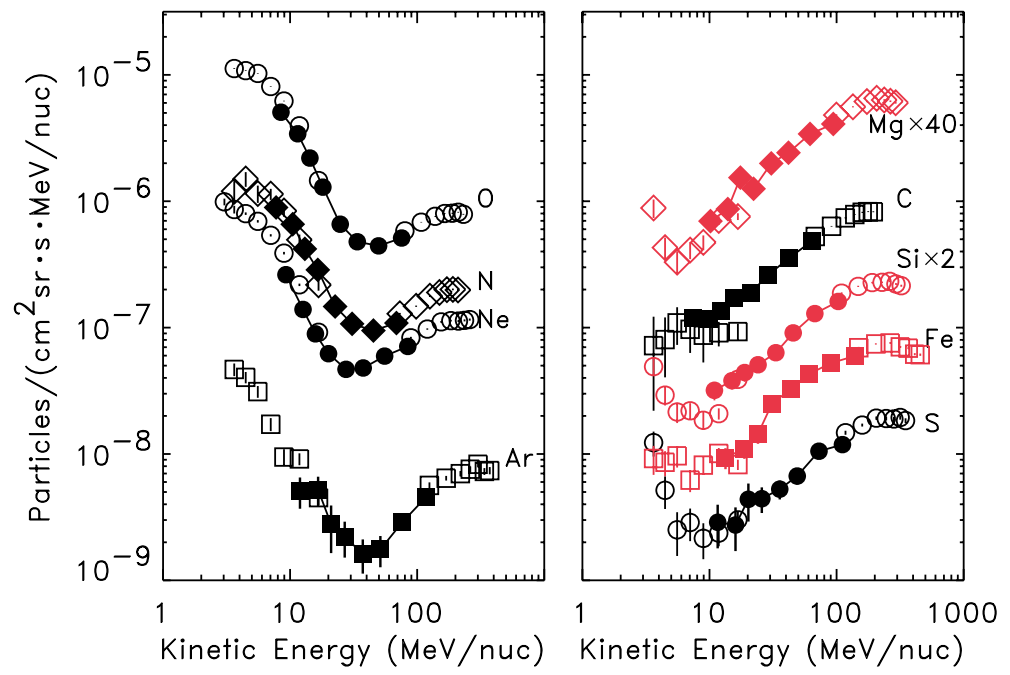

Fig. 17 Solar minimum spectra showing ACR contributions to $\mathrm{N}, \mathrm{O}, \mathrm{Ne}$, and Ar (left side), along with similar, but much smaller contributions to the $1 \mathrm{AU}$ spectra of five other species (right side). The data are from ACE and Wind (from Leske et al. 2000)

Fig. 18 Time history of ACR oxygen from 1968 to 2006 , based on measurements from OGO-5, IMP-7\&8, SAMPEX, and ACE. Also shown is the Climax neutron monitor count rate, scaled to the 25 th power. The expected profile for 2007-2010 is shown in yellow

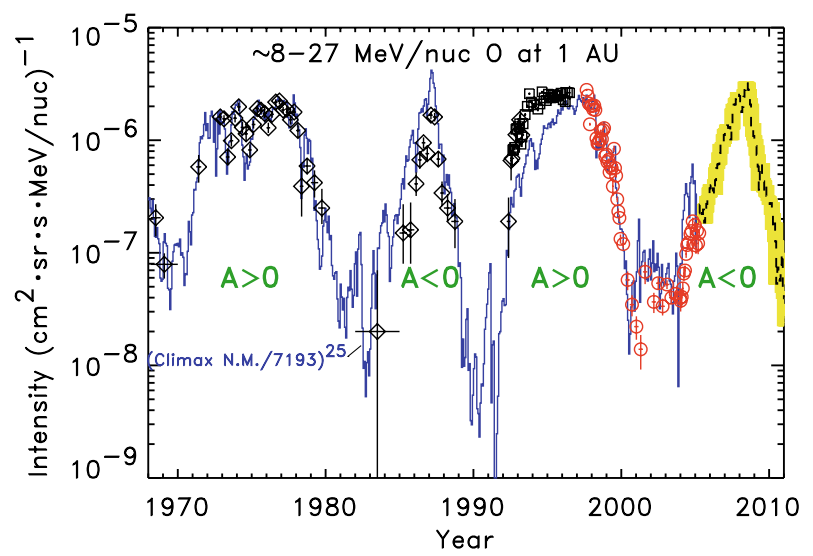

It has been suggested that these additional rare-ACR species could originate from the "inner source" of pickup ions associated with dust grains near the Sun (e.g., Cummings et al. 2002) or from an "outer source" of pickup ions originating in the Kuiper Belt (Schwadron et al. 2002). Cummings et al. (2002) also found that the energy spectra of ACR Mg, Si, and S measured at 1 AU with the Wind spacecraft (Reames 1999) did not fit the modulation model using the same modulation parameters that fit the more abundant ACR elements. This puzzle and the mystery of the origin of the rare ACRs will be addressed during the 20072008 solar minimum, when the intensities are expected to reach a maximum (see Fig. 18), using the LET and HET sensors in combination with instruments on ACE. LET and HET complement the SIS and CRIS instruments on ACE by providing coverage that extends to lower energy and by making much better measurements of $\mathrm{H}$ and $\mathrm{He}$ spectra.

If the minor ACR species $(\mathrm{C}, \mathrm{Na}, \mathrm{Mg}, \mathrm{Si}, \mathrm{S}$, and $\mathrm{Fe}$ ) do originate from pickup ions formed from Kuiper Belt material, then the source of these pickup ions is much more confined to 
the ecliptic than are interstellar pickup ions. The difference in the latitude distribution of the Kuiper Belt and interstellar pickup ion sources might be expected to result in a solar-cycle dependence in the ratio of minor-ACR species to major-ACR species, which has, to our knowledge, not yet been modeled.

\section{Instrumentation}

\subsection{Design Requirements}

To achieve the objectives discussed in Sect. 2 the LET sensor had to satisfy several design requirements. These were ultimately derived from the STEREO Mission Requirements Document (taking into account allocated resources), which states that the SEP suite shall measure the intensity, composition, energy spectra, and direction of energetic protons from 0.06 to $40 \mathrm{MeV}$, heavier ions from $\sim 0.03$ to $40 \mathrm{MeV} /$ nucleon, electrons from $\sim 0.03$ to $6 \mathrm{MeV}$, and ${ }^{3} \mathrm{He}$-rich solar particle events. The STEREO Level-1 Requirements Document states that:

LET shall measure SEP ion fluxes, spectra, and composition in two oppositely directed $100 \times 30 \mathrm{deg}$ or better FOVs, covering the energy range from 3-25 MeV/ nucleon for $\mathrm{C}$ to $\mathrm{Fe}$ ions, $1.5-13 \mathrm{MeV} /$ nucleon for $\mathrm{He}$, and 1.5-3 MeV for $\mathrm{H}$. The mass resolution shall be better than 0.35 amu for ${ }^{3} \mathrm{He}$ and ${ }^{4} \mathrm{He}$. Time resolution shall be 1 minute for $\mathrm{H}$ and $\mathrm{He}$ Beacon data, and 15 minutes otherwise. LET shall handle at least 1,000 events/sec.

The above statement has led to the requirements and goals summarized in Table 3, which were used to drive the detailed design of the instrument. Table 3 also shows the present capability of the two LET instruments, which meet or surpass essentially all of these requirements.

\subsection{Approach}

The LET instrument employs the well-established $\mathrm{d} E / \mathrm{d} x$ vs. total energy technique to determine the nuclear charge, and in some cases the mass, of detected heavy ions. This particleidentification technique uses the energy loss signal from a detector that the particle fully penetrates $(\Delta E)$ and the energy deposited in a following detector in which the particle stops $\left(E^{\prime}\right)$, together with the thickness penetrated in the $\Delta E$ detector $(L)$ to obtain an estimate of the particle's charge, $Z$, as follows (Stone et al. 1998b):

$$
Z \cong\left(\frac{k}{L(2+\varepsilon)^{a-1}}\right)^{1 /(a+1)}\left(E^{a}-E^{\prime a}\right)^{1 /(a+1)} .
$$

Here $E=\Delta E+E^{\prime}, 2+\varepsilon$ is the mass-to-charge ratio of the nuclide being considered, and $k$ and a are constants obtained by approximating the range-energy relation for heavy ions in silicon in the form $R \cong k\left(M / Z^{2}\right)(E / M)^{a}$, where $R$ and $M$ denote the particle range and mass, respectively. In the energy range covered by LET $a \cong 1.55$ and $k \cong 18.6$ when $R$ is expressed in $\mu \mathrm{m}$ of $\mathrm{Si}$ and $E / M$ in $\mathrm{MeV} /$ nucleon. In the course of data analysis these values are adjusted to fit the observed relationship between $\Delta E$ and $E^{\prime}$. 
Table 3 LET design requirements

\begin{tabular}{|c|c|c|c|}
\hline Description & Requirement & Goal & Capability \\
\hline Field of view & $\begin{array}{l}2 \text { oppositely directed } \\
100^{\circ} \times 30^{\circ} \text { fans }\end{array}$ & $\begin{array}{l}2 \text { oppositely directed } \\
130^{\circ} \times 30^{\circ} \text { fans }\end{array}$ & $\begin{array}{l}2 \text { oppositely directed } \\
133^{\circ} \times 29^{\circ} \text { fans }\end{array}$ \\
\hline $\begin{array}{l}\text { Energy range } \\
\text { (MeV/nucleon) }\end{array}$ & $\begin{array}{l}\mathrm{H}: 1.8-3 ; \mathrm{He}: 1.8-13 \\
\mathrm{O}: 4-25 ; \mathrm{Fe}: 4-25\end{array}$ & $\begin{array}{l}\mathrm{H}: 1.4-6 ; \mathrm{He}: 1.4-13 \\
\mathrm{O}: 2.5-25 ; \mathrm{Fe}: 2.5-25\end{array}$ & $\begin{array}{l}\mathrm{H}: 1.8-12 ; \mathrm{He}: \\
1.8-15 \mathrm{O}: 4-50 ; \\
\text { Fe: } 4-50\end{array}$ \\
\hline $\begin{array}{l}\text { Geometry factor } \\
\left(\mathrm{cm}^{2} \mathrm{sr}\right)\end{array}$ & $\begin{array}{l}\text { H, He: } 0.5 \\
6 \leq Z \leq 26: 2\end{array}$ & $\begin{array}{l}\text { H, He: } 0.9 \\
6 \leq Z \leq 26: 4.5\end{array}$ & $\begin{array}{l}\mathrm{H}, \mathrm{He}: 4.04 \\
6 \leq Z \leq 26: 4.04\end{array}$ \\
\hline L1 noise level & $<90 \mathrm{keV} \mathrm{rms}$ & $\leq 60 \mathrm{keV} \mathrm{rms}$ & $\begin{array}{l}<30 \mathrm{keV} \mathrm{rms} \mathrm{for} \\
25 \mu \mathrm{m} \text { thick L1 }\end{array}$ \\
\hline $\begin{array}{l}\text { L1 thickness } \\
\text { uniformity }\end{array}$ & $\sigma_{T} \leq 0.6 \mu \mathrm{m} \mathrm{rms}$ & $\sigma_{T} \leq 0.3 \mu \mathrm{m} \mathrm{rms}$ & $\begin{array}{l}\sigma_{T}=0.44 \mu \mathrm{m} \mathrm{rms} \\
\text { (typical) }\end{array}$ \\
\hline $\begin{array}{l}\text { Element } \\
\text { resolution }\end{array}$ & $\begin{array}{l}\text { Resolve: } \mathrm{H}, \mathrm{He}, \mathrm{C}, \mathrm{N} \text {, } \\
\mathrm{O}, \mathrm{Ne}, \mathrm{Mg}, \mathrm{Si}, \mathrm{Fe}\end{array}$ & $\begin{array}{l}\text { Also resolve: } \mathrm{Na}, \mathrm{Al} \text {, } \\
\mathrm{Ar}, \mathrm{Ca}\end{array}$ & $\begin{array}{l}\text { Resolve: } \mathrm{H}, \mathrm{He}, \mathrm{C}, \mathrm{N} \text {, } \\
\mathrm{O}, \mathrm{Ne}, \mathrm{Na}, \mathrm{Mg}, \mathrm{Al}, \\
\mathrm{Si}, \mathrm{S}, \mathrm{Ar}, \mathrm{Ca}, \mathrm{Fe}, \mathrm{Ni}\end{array}$ \\
\hline $\begin{array}{l}{ }^{4} \text { He mass } \\
\text { resolution }\end{array}$ & $\leq 0.35 \mathrm{amu}$ & $\leq 0.25 \mathrm{amu}$ & $\begin{array}{l}\text { L1L2: } 0.23-0.33 \\
\text { L1L2L3: } 0.13-0.16\end{array}$ \\
\hline $\begin{array}{l}\text { Maximum event } \\
\text { rate }\end{array}$ & 1,000 per sec & 5,000 per sec & $1,000-2,000$ per sec \\
\hline Energy binning & $\begin{array}{l}6 \text { intervals per species } \\
\text { for } Z \geq 2 ; 3 \text { for } H\end{array}$ & $\begin{array}{l}8 \text { intervals per species } \\
\text { for } Z \geq 2 ; 3 \text { for } H\end{array}$ & $\begin{array}{l}\sim 10 \text { intervals per } \\
\text { species for } H \text { and } \\
Z \geq 2\end{array}$ \\
\hline $\begin{array}{l}\text { Onboard species } \\
\text { identification }\end{array}$ & $\begin{array}{l}\mathrm{H}, \mathrm{He}, \mathrm{C}, \mathrm{N}, \mathrm{O}, \mathrm{Ne} \\
\mathrm{Mg}, \mathrm{Si}, \mathrm{Fe}\end{array}$ & Add ${ }^{3} \mathrm{He}, \mathrm{S}, \mathrm{Ar}, \mathrm{Ca}$ & $\begin{array}{l}\mathrm{H},{ }^{3} \mathrm{He},{ }^{4} \mathrm{He}, \mathrm{C}, \mathrm{N}, \mathrm{O}, \\
\mathrm{Ne}, \mathrm{Na}, \mathrm{Mg}, \mathrm{Al}, \mathrm{Si}, \mathrm{S}, \\
\mathrm{Ar}, \mathrm{Ca}, \mathrm{Fe}, \mathrm{Ni}\end{array}$ \\
\hline Time resolution & $\begin{array}{l}\mathrm{H}, \mathrm{He}: 1 \min Z \geq 6 \text { : } \\
15 \mathrm{~min} \text { Telemeter } \\
1 \text { event/s }\end{array}$ & $\begin{array}{l}\mathrm{H}, \mathrm{He}: 1 \min Z \geq 6 \text { : } \\
1 \text { min Telemeter } \\
4 \text { events/sec }\end{array}$ & $\begin{array}{l}\mathrm{H}, \mathrm{He}: 1 \min Z \geq 6 \text { : } \\
1 \text { min Telemeter } \\
4 \text { events/s }\end{array}$ \\
\hline Beacon telemetry & $\begin{array}{l}1 \min \text { for } \mathrm{H}, \mathrm{He}, \\
Z \geq 6\end{array}$ & $\begin{array}{l}1 \min \text { for } \mathrm{H}, \mathrm{He}, \\
Z \geq 6\end{array}$ & $\begin{array}{l}1 \text { min for } \mathrm{H}, \mathrm{He}, \\
Z \geq 6\end{array}$ \\
\hline
\end{tabular}

\subsection{The LET Sensor System}

The sensor system is illustrated with a three-dimensional drawing in Fig. 19 and in crosssection in Fig. 20a. The active elements are all silicon solid-state detectors and include three different detector designs, designated L1, L2, and L3. Table 4 lists the nominal characteristics of each of these detector designs. The geometry used for segmenting the detectors into multiple active elements is illustrated in Fig. 20b.

The instrument has 10 entrance apertures, each occupied by an L1 detector. Five of these are arranged along an arc of a circle that in flight lies in the ecliptic plane and is centered along the direction of the nominal Parker spiral magnetic field, generally toward the Sun. The other five are located $180^{\circ}$ from these, centered on the same direction but facing generally away from the Sun. The central area of the instrument contains four additional detectors, 


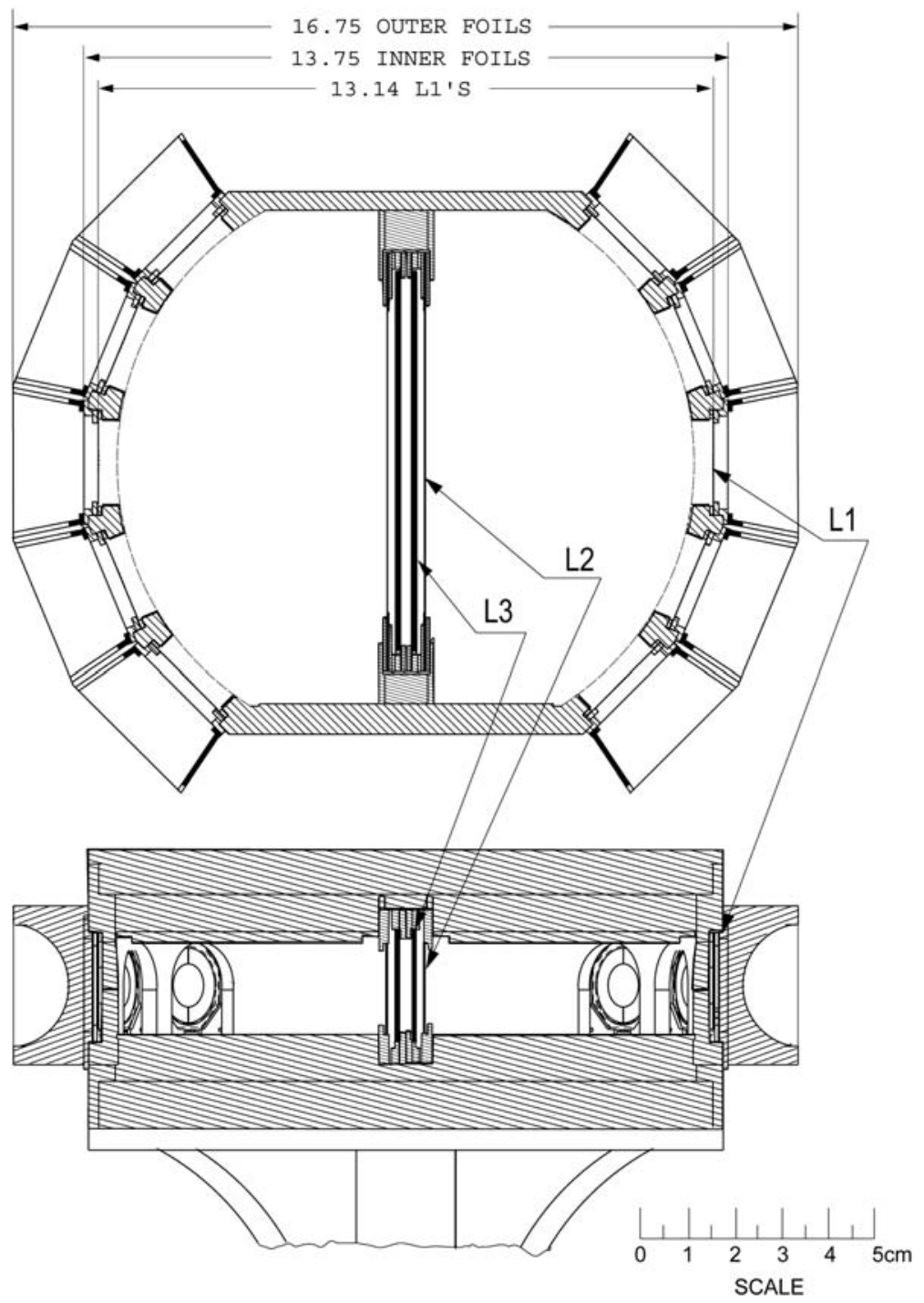

Fig. 19 Two cut-away views of the LET sensor system illustrate the locations of the detectors, entrance foils, and collimators, as well as structural components

including two L2 and two L3 devices. Each detector is segmented into multiple active areas, as indicated in Table 4. This provides some position sensitivity, which is used for determining particle trajectories, as well as for reducing noise and improving instrument performance when exposed to high intensities of incident particles. Each segment of each detector (a total of 54 signal sources) is separately pulse height analyzed to obtain a measure of the energy deposited by the particle in that segment.

The sunward- and antisunward-facing halves of the detector array are designated the " $\mathrm{A}$ " and "B" sides of the instrument, respectively. The labels used to designate the various detector segments are shown in Fig. 20. Coincidence between the L1 and L2 detectors defines 


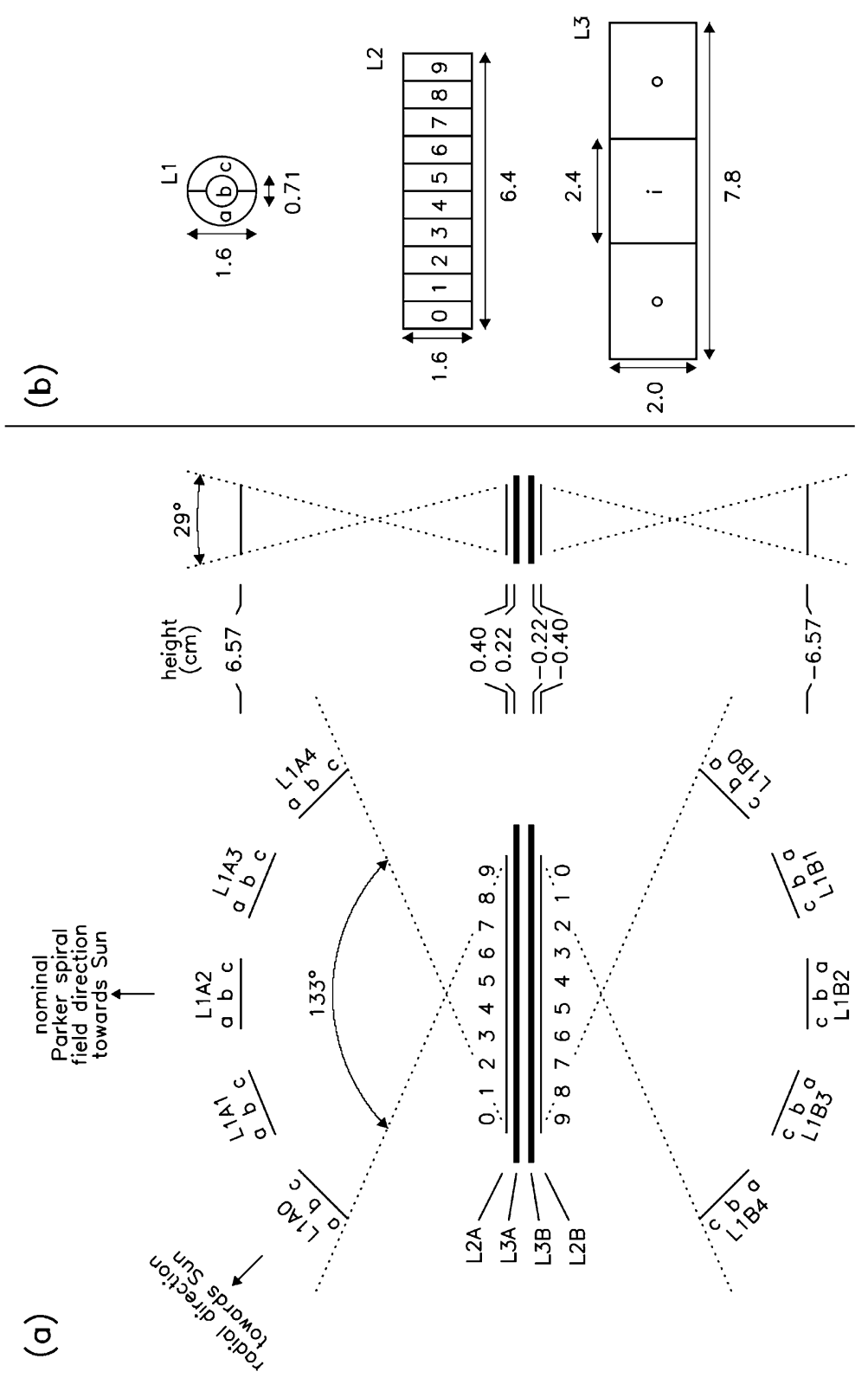

일

元

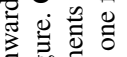

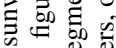

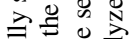

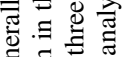

品言萿

的岁这

동 웡

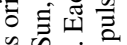

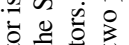

造递

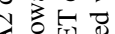

乐乐

을

踏

完摸

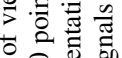

은월

త

छ

O

当

它

की

专要

牙寻

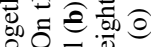

过

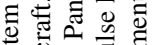

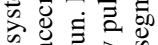

कै की

可哥

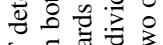

도을

뭉을

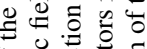

पै

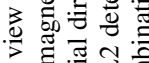

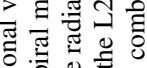

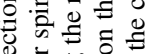

它

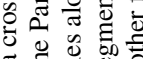

吃氜

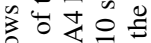

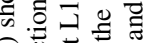

ఏ已

๑

๘ สึ

งิ

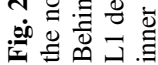


Table 4 Detector design characteristics

\begin{tabular}{llccc}
\hline $\begin{array}{l}\text { Detector } \\
\text { designation }\end{array}$ & $\begin{array}{l}\text { Number in LET } \\
\text { instrument }\end{array}$ & $\begin{array}{l}\text { Thickness } \\
(\mu \mathrm{m})\end{array}$ & $\begin{array}{l}\text { Active area } \\
\left(\mathrm{cm}^{2}\right)\end{array}$ & $\begin{array}{l}\text { Active segments } \\
\text { per detector }\end{array}$ \\
\hline L1 & 10 & 24 & 2.0 & 3 \\
L2 & 2 & 50 & 10.2 & 10 \\
L3 & 2 & 1000 & 15.6 & $2^{*}$ \\
\hline
\end{tabular}

* The two outer regions of each L3 detector are connected to the same PHA channel

the instrument field of view comprising two approximately fan-shaped regions extending $\pm 14.5^{\circ}$ normal to the ecliptic and $\pm 66.5^{\circ}$ about the nominal Parker spiral field direction within the ecliptic plane (Fig. 20). The disk-shaped detector assembly is mounted between the LET electronic boards yielding a compact design that has relatively short signal connections between detectors and front-end electronics and in which the electronic boards and housing provide a degree of shielding against out-of-geometry particles.

Nuclei that enter the instrument through one of the L1 detectors, penetrate into the following L2 detector, and subsequently come to rest in that L2 detector or in one of the two L3 detectors that are the most useful for charge identification. Using the signal from the detector in which the particle stops as $E^{\prime}$ and the signal from the preceding detector as $\Delta E$ one obtains a set of distinct tracks, one for each nuclide, on a plot of $\Delta E$ vs. $E^{\prime}$. Figure 21 illustrates the nominal response tracks (solid lines) for selected nuclides stopping at various depths in the LET instrument for the case where the particles penetrate the $\Delta E$ detector normal to its surface $\left(\theta=0^{\circ}\right)$.

The measured signals will be displaced slightly from the nominal track positions when the particle penetrates the $\Delta E$ detector at some larger angle, $\theta$, or at a position where the detector thickness differs from the nominal value, $L_{0}$. Approximate information about the angle of incidence is available since the pulse height data indicate which detector segments the particle passed through. Detector thickness maps derived from laboratory measurements (see Sect. 3.4) or from flight data can be used to better estimate the thickness at the point penetrated. If $f=L / L_{0}$ is the ratio of the actual thickness penetrated to the nominal detector thickness (combining the effects of both the incidence angle and the detector thickness nonuniformity), then the measured $\Delta E$ and $E^{\prime}$ signals can be scaled so that they lie on the track corresponding to the nominal detector thickness, $L_{0}$, and $\theta=0^{\circ}$. Examination of (1) shows that dividing $E^{\prime}$ and $\Delta E$ by $f^{1 / a}$ accomplishes the desired transformation (recall that $E=\Delta E+E^{\prime}$ ). Figure 22 illustrates this procedure using the results of a Monte Carlo simulation of the LET response to an isotropic distribution of particles having power-law energy spectrum with intensity proportional to $E^{-2}$. In the upper left panel the uncorrected measurements of $\Delta E$ obtained from L1 vs. $E^{\prime}$ from L2 are shown for particles stopping in L2. These data, corrected for the mean thickness of the penetrated L1 segment and for the mean angle between the hit segments in L1 and L2, are plotted in the upper right panel of Fig. 22. Corresponding uncorrected and corrected plots for particles stopping in L3 are shown in the lower panels. This correction technique is the basis for the onboard particle identification performed by LET.

To obtain energies of the incident particles, corrections must be applied to the sum of the measured $\Delta E$ and $E^{\prime}$ energies to account for unmeasured energy losses in overlying material. This material includes any detectors preceding the $\Delta E$ detector as well as a pair of thin windows mounted in front of each L1 detector to keep the sensor assembly light tight and to provide a degree of protection from micrometeorites. The energy losses in the 

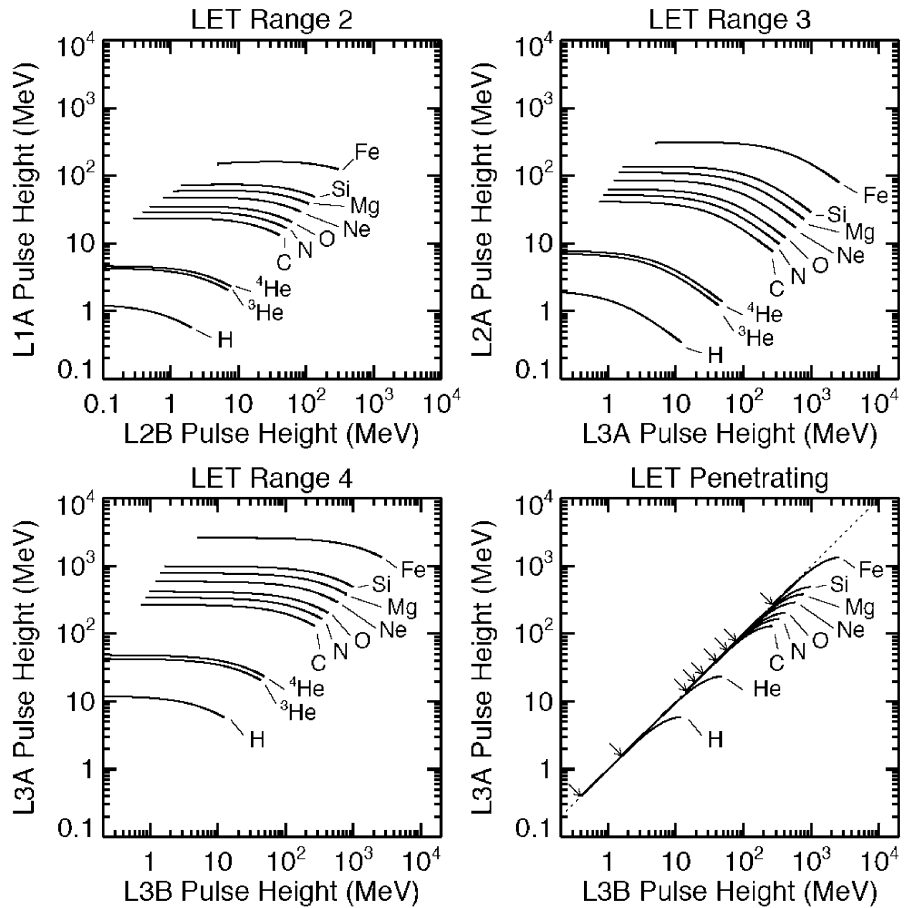

Fig. 21 Ideal response tracks are shown for selected energetic ion species normally incident on a stack of detectors of thicknesses $24 \mu \mathrm{m}$ (L1A2), $50 \mu \mathrm{m}$ (L2A), 1,000 $\mu \mathrm{m}$ (L3A), and 1,000 $\mu \mathrm{m}$ (L3B). Ranges 2, 3 , and 4 include particles that stop in L2A, L3A, and L3B, respectively. On the "penetrating" panel, which includes particles energetic enough to exit through the back surface of L3B, arrows indicate the high-energy end points of the various element tracks. Note that the tracks that are plotted show the average response and do not include fluctuations due, for example, to energy loss fluctuations or uncorrected variations of incidence angle (see Fig. 22)

detectors preceding the $\Delta E$ detector are directly measured, while the energy loss in the windows (consisting of two layers of $8-\mu \mathrm{m}$ thick Kapton foil, which together contribute an energy loss equivalent to that in $\sim 12 \mu \mathrm{m}$ of $\mathrm{Si}$ ) is calculated by scaling from the energy loss measured in L1.

Because of the importance of the isotope ${ }^{3} \mathrm{He}$ for identifying material from impulsive solar particle events (see Sect. 2.2), LET is designed to resolve ${ }^{3} \mathrm{He}$ from ${ }^{4} \mathrm{He}$ over much of the measured energy range. Tracks for different isotopes of an element are displaced slightly from one another on a $\Delta E$ vs. $E^{\prime}$ plot, corresponding to the fact that the quantity $\varepsilon$ in (1) depends on the particle's mass-to-charge ratio $(\varepsilon=M / Z-2)$. Figure 21 illustrates the difference in the nominal response tracks for the isotopes ${ }^{3} \mathrm{He}$ and ${ }^{4} \mathrm{He}$. In addition to ${ }^{3} \mathrm{He}$ and ${ }^{4} \mathrm{He}$, a few other key isotope pairs (e.g., ${ }^{20} \mathrm{Ne}$ and ${ }^{22} \mathrm{Ne}$ ) should be distinguishable in the upper portion of the LET energy range where the resolution is best.

The response tracks are broadened by the finite resolution of the instrument, which is attributable to a number of different effects (discussed in detail by Stone et al. 1998c). Typically the most significant contributions to the uncertainties in of the charge and mass determinations of $\mathrm{d} E / \mathrm{d} x$ vs. total energy instruments are attributable to the statistical nature of the energy-loss process (Landau fluctuations) and to errors in $L$, the thickness of material penetrated in the $\Delta E$ detector due to uncertainties the angle of incidence and to uncorrected 

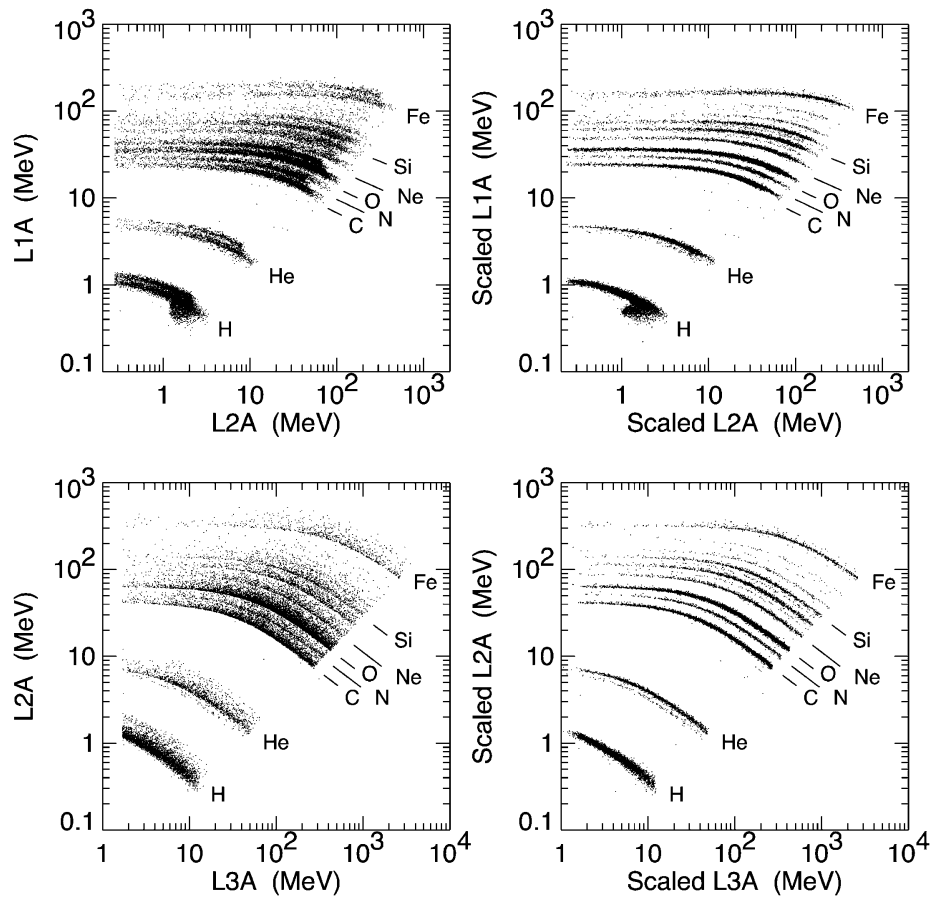

Fig. 22 Monte Carlo simulation of the LET response to particles incident from the A side and stopping in the L2A (upper panels) or L3A (lower panels) detectors. The left-hand panels show the actual energy losses while the right-hand panels show the same events after approximate correction for variations in incidence angle and detector thickness, as described in the text. The simulation includes the effects of incidence angle variations, L1 detector thickness variations (based on the measured detector characteristics for the A-side detectors on the Ahead spacecraft), Bohr/Landau fluctuations in the particle energy losses, and electronic noise. An incident particle population with typical gradual event composition was assumed, except that $\mathrm{H}$ and $\mathrm{He}$ were suppressed by factors of 5,000 and 500, respectively, and ${ }^{3} \mathrm{He}$ was set to be $10 \%$ of ${ }^{4} \mathrm{He}$. The multiple tracks for individual elements in the upper left panel are due to the differences among the thicknesses of the five L1A detectors

nonuniformities in the detector thickness. The charge and mass resolution were determined from a combination of Monte Carlo simulations and accelerator calibrations, which are discussed in Sect. 4.5 and Sect. 4.2, respectively.

\subsection{Silicon Detectors}

Photographs of the three different detector designs used in LET are shown in Fig. 23. All of the detectors were manufactured by Micron Semiconductor Ltd. starting from highresistivity float-zone-refined $n$-type silicon wafers with $\langle 100\rangle$ crystal orientation. These wafers were lapped and polished to the desired detector thicknesses. This mechanical thinning to produce the very thin, fragile wafers needed for the L1 detectors had a relatively poor yield and led to the use of detectors with thicknesses ranging from 22 to $30 \mu \mathrm{m}$ (average $24 \mu \mathrm{m}$, see Table 4 and Appendix 1), somewhat thicker than the original design goal of $20 \mu \mathrm{m}$.

Boron ions were implanted on one surface to produce pn junctions in the desired pattern of active elements, as illustrated in Fig. 20b. Around the periphery of the active area, thin 


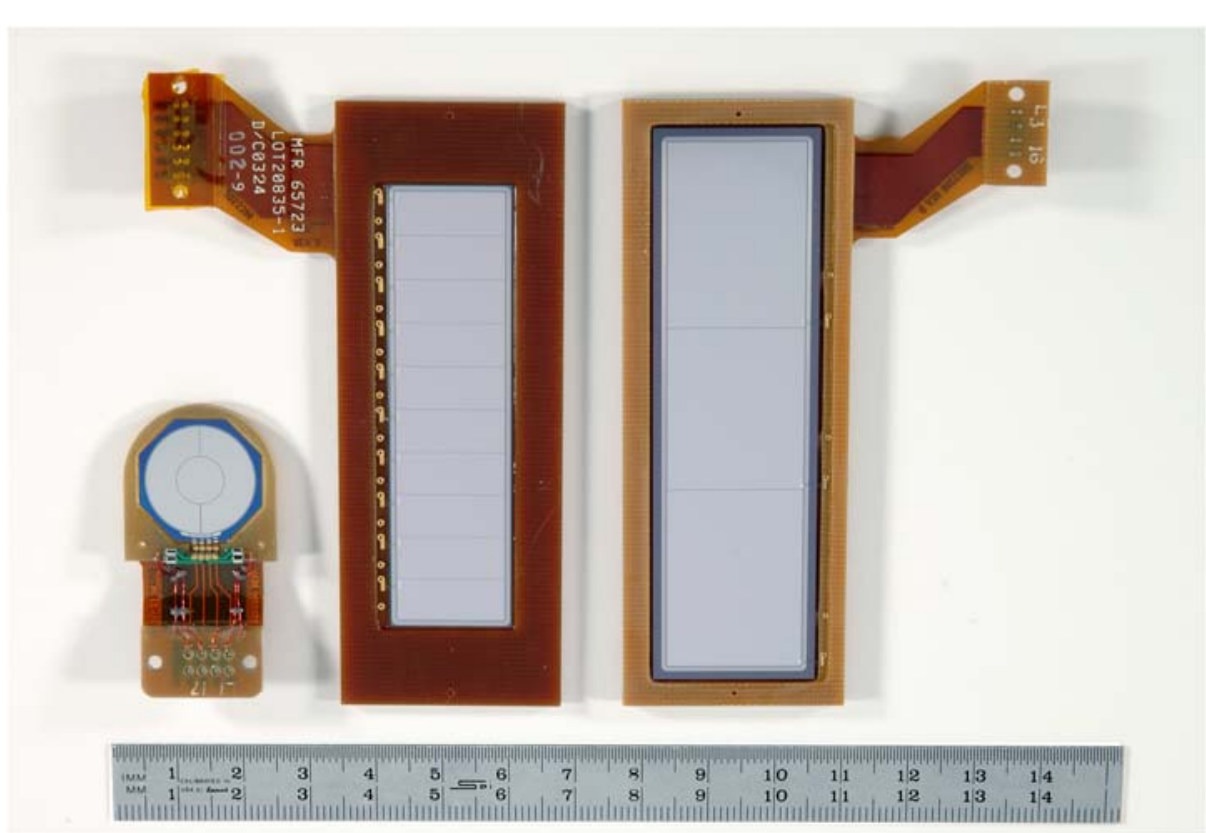

Fig. 23 Photographs showing (from left to right) examples of L1, L2, and L3 detectors. These views from the junction surfaces of the detectors show the segmentation into multiple active areas. The opposite (ohmic) surfaces consist of a single, full-area contact in each detector design. Detectors are installed by the manufacturer in multilayer circuit board mounts with flexible metallized Kapton strips for making electrical connections to the LET bias and pulse height analysis circuitry

guard rings were implanted to help avoid regions of high electric field that could increase the detector noise. On the opposite surface of the detector, phosphorus was implanted to form a single ohmic contact. Aluminum was subsequently evaporated over the implanted areas on each surface. Finally, a thin layer of $\mathrm{SiO}_{2}$ was deposited over the detector surfaces to protect against surface contamination. This $\mathrm{SiO}_{2}$ was omitted on the flight $\mathrm{L} 3$ detectors because thermal-vacuum tests (discussed later) suggested that it was contributing to a long-term instability of the leakage current in these thicker detectors, which are operated at relatively high bias voltages.

The silicon detector chips were installed in custom-designed detector mounts using a silicone resin (Shin-etsu KJR-9022E). These mounts were fabricated as multilayer FR4 or polyimide circuit boards including a flexible Kapton circuit layer on which the signals are brought out to a connector that could be plugged into the circuit board containing the pulse height analysis electronics (see Sect. 3.5.1). Connections between the active detector elements and the associated pads on the detector mount were made using redundant sets of wirebonds made with $25-\mu \mathrm{m}$-diameter aluminum wire. Pull tests were performed to assure good bond quality.

Electrical and environmental tests were performed to qualify individual detectors for flight prior to assembly of the flight instruments. Measurements of detector capacitance vs. bias voltage (CV test) and leakage current vs. bias voltage (IV test) established the voltages at which each detector becomes fully depleted and breaks down, respectively. For the L3 and L2 detectors, maps of pulse height vs. position on the detector were made using a collimated source of $5.8 \mathrm{MeV}$ alpha particles from a ${ }^{244} \mathrm{Cm}$ source. Since the range of the 
alpha particles ( $\sim 33$ microns of $\mathrm{Si}$ ) is less than the L2 and L3 detector thicknesses, one should obtain a narrow pulse height distribution that is consistent across the detector when the ionization charge is being collected with high efficiency. With the alpha particles incident on the detector from the ohmic surface these measurements are sensitive to the local depletion characteristics of the detector, which generally vary from point to point because of nonuniformity in the doping of the silicon crystals from which the detectors were fabricated. Response maps made at a series of bias voltages were used to determine the minimum bias at which the detector was fully depleted over its entire area. The depletion voltage determined in this way is consistently higher than the average depletion voltage obtained from the CV test. In flight the detectors are operated at fixed bias voltages of $+5 \mathrm{~V},+10 \mathrm{~V}$, and $+175 \mathrm{~V}$ for L1, L2, and L3, respectively.

All of the LET detectors were subjected to a thermal-vacuum stability test in which they were exposed to temperature extremes of $-25^{\circ} \mathrm{C}$ and $+40^{\circ} \mathrm{C}$. During the test the detectors were continuously biased and their leakage current and noise were monitored. The test included an extended period (typically $>3$ weeks) at the warm temperature extreme, which was intended to uncover latent instabilities in the detectors. These tests demonstrated a long-term leakage current growth in L3 detectors that were made with a protective $\mathrm{SiO}_{2}$ coating (discussed earlier) and led to the replacement of all of the flight L3 detectors with devices that did not have this coating.

Prior to delivery from the manufacturer the detectors were subjected to a random vibration test as part of their qualification. Because the L1 detectors are very thin $(20-30 \mu \mathrm{m})$, microscopic chipping of their edges, which can occur when detectors are sawed out of a larger silicon wafer, can lead to cracks that may propagate into the active area of the device when subjected to mechanical stress. Before the final assembly of the flight instruments the L1 detectors were put through an acoustic screening test to eliminate devices that could subsequently fail in this way.

A precise knowledge of detector thicknesses is needed in order to correct the $\Delta E$ and $E^{\prime}$ signals so that measurements from all combinations of detector segments can be mapped onto a common response track (see Sect. 3.3). For the L1 detectors, thickness maps were made by using a collimated beam of $8.78 \mathrm{MeV}$ alpha particles from a ${ }^{228} \mathrm{Th}$ source. A thick detector placed behind the L1 was used to measure the residual energy of the alpha particles that passed through this thin device at each of 12 points distributed over its surface. These residual energy values were translated into detector thicknesses by comparison with measurements on a set of thin aluminum foils of known thickness. The left-hand panel of Fig. 24 shows a typical L1 thickness map obtained in this way. The right-hand panel shows the measured correlation between the L1 thicknesses obtained from the alpha particle maps and detector capacitances measured with the devices fully depleted. Since the area of each segment is known (Table 4) and capacitance scales inversely with thickness for a simple parallel-plate capacitor, this type of correlation can be used as the basis for estimating the thicknesses of detectors that are too thick to be measured using available alpha particle sources. Appendix 1 summarizes the thickness measurements that were made for all of the flight L1 detectors.

Preliminary measurements of thicknesses for some of the L2 and L3 detectors were derived from data obtained with energetic heavy ions during a cyclotron calibration run (see Sect. 4.2). Such data are not available for all of the flight detectors because some devices were replaced subsequent to the cyclotron run. An initial estimate of the relative thicknesses of the unmapped L2 detector segments was obtained from laboratory measurements of the capacitances of individual detector elements made with the detector fully depleted (cf. Fig. 24 right panel). 

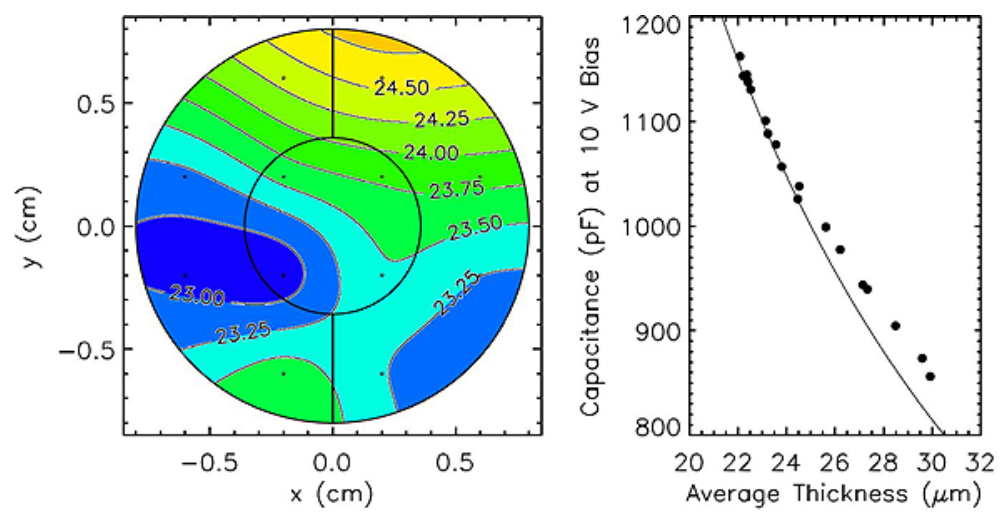

Fig. 24 Left-hand panel shows the thickness map of one of the L1 detectors obtained by measuring the residual energy of a collimated beam of alpha particles incident at 12 different locations centered on the positions indicated by the small circles. Thickness contours are labeled in $\mu \mathrm{m}$. Right-hand panel shows the measured correlation between average L1 detector thickness and detector capacitance for the 20 flight L1 detectors. For these measurements all three detector segments were combined. The line indicates the ideal capacitance vs. thickness relationship expected from a silicon detector with the $2 \mathrm{~cm}^{2}$ active area of an L1 device and a thickness equal to the plotted physical thickness minus a total of $3 \mu \mathrm{m}$ dead thickness, which could be due to conductive layers on the two surfaces caused by ion implantation and subsequent diffusion during annealing. The tight correlation between thickness and detector capacitance was used to obtain estimates of the segment-to-segment thickness variation of L2 detectors, which were too thick to be measured with available alpha particle sources

Heavy ion data collected in flight will be used to obtain final thickness values for all of the L2 and L3 detector segments. As is clear in the simulations shown in Fig. 22, H, He, and several of the heavier elements are unambiguously identifiable on a plot of $\Delta E$ vs. $E^{\prime}$ even without applying corrections for incidence angle and detector thicknesses; most of the abundant heavy ions in ranges 3 and 4 become well-resolved when corrected for incidence angle using a nominal detector thickness. To refine the estimate of a detector thickness, (1) is solved for $L$ and evaluated using measured $\Delta E$ and $E^{\prime}$ values in flight data selected for a given species with known $Z$.

\subsection{Electronics}

The SEP suite is organized as four distinct instruments serviced by a block of common electronics, "SEP Central", that includes low voltage and detector bias supplies, as well as a microprocessor that coordinates data and command flow, and provides a single-point interface with the IMPACT DPU (see Fig. 2). Three of the four instruments contain microprocessors in close contact with the detector front-end electronics to support onboard high-speed identification of particle species and energy. Very tight power and mass constraints inspired two innovations: a custom designed pulse height analysis ASIC and a unique FPGA-embedded microprocessor based on the Minimal Instruction Set Computer (MISC) architecture. The custom ASIC, used in both LET and HET, and the unique processors used in LET, HET, SIT, and SEP Central, are discussed in detail in the following sections.

\subsubsection{The PHASIC}

The silicon detectors in LET are read out using a Pulse Height Analysis System Integrated Circuit (PHASIC). The PHASIC was custom designed for the STEREO mission 
Fig. 25 Photo of the PHASIC hybrid. The package dimensions are $31.8 \mathrm{~mm} \times 31.8 \mathrm{~mm}$. The PHASIC chip (located in the center) has dimensions of $7.4 \mathrm{~mm} \times 7.4 \mathrm{~mm}$

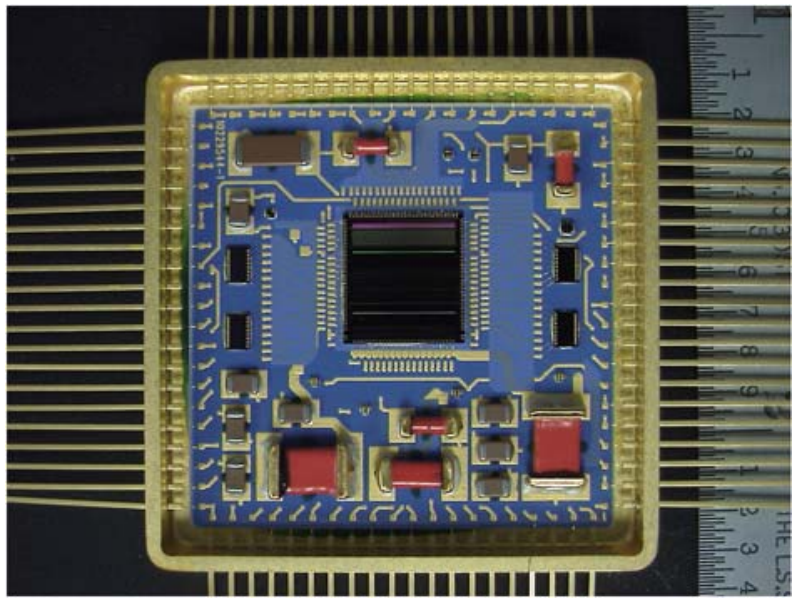

and contains 16 identical channels of pulse height analysis (PHA) circuitry. The PHASIC represents an evolution of similar PHA designs which were flight-proven in numerous space instruments over the past 40 years (Halpern et al. 1968; Halpern and Marshall 1968; Harrington and Marshall 1968, 1969; Harrington et al. 1974; Althouse et al. 1978; Cook et al. 1993; Stone et al. 1998b, 1998c). The key technical development is the use of CMOS rather than bipolar technology and the inclusion of all passive components on the ASIC.

Relative to the prior design employed in the SIS and CRIS instruments on ACE (Stone et al. 1998b, 1998c) the PHASIC performance is improved in several areas: (1) board space is reduced by a factor of 16 (see picture in Fig. 25), (2) power consumption is lower by a factor of 4, and (3) dynamic range is larger by a factor of 5. The very wide dynamic range of $\sim 10,000: 1$ is achieved with a dual gain approach. Each preamplifier output signal is coupled to two shaping amplifier/offset-gate/peak-detector/Wilkinson-ADC chains that operate in parallel, but with gains that differ by a factor of 20 . The separate low and high gain on-chip Wilkinson-ADCs each provide 11-bit resolution and excellent differential linearity. The system is designed for positive-polarity input signals.

The PHASIC supports high rate, low deadtime analysis via bipolar shaping with a primary time constant of $1 \mu \mathrm{s}$, time to peak of $1.9 \mu \mathrm{s}$, and a $32 \mathrm{MHz}$ clock rate for the Wilkinson-ADCs. In addition, on-chip digital circuitry provides sparsified parallel readout of ADC conversion results, and control of the PHAs.

The PHASIC offers a high degree of programmable reconfigurability that allows a single design to accommodate a wide range of different detector capacitances, leakage currents, and signal ranges. Programmable items include: the preamplifier gain (feedback capacitance), the preamplifier input FET transconductance, and the peak detection threshold. The preamplifiers are DC coupled to the detectors and a 10-bit current DAC is provided on-chip at each preamplifier input to aid in balancing and measuring the detector leakage current. A built-in precision test pulser for each dual-gain PHA chain is used for functional test and calibration.

The PHASIC chip was fabricated using American Microdevices Inc.'s 0.5- $\mu \mathrm{m}$ CMOS process $(\mathrm{C} 5 \mathrm{~N})$ incorporating high-density linear capacitors and high value resistors suitable for mixed-signal designs. For STEREO applications the chip and supporting passive components are mounted on a ceramic substrate and installed in a standard 80-pin Kovar package to form a "PHASIC" hybrid. Prototype tests place the total dose tolerance at 
Table 5 PHASIC chip specifications

\begin{tabular}{ll}
\hline Number of dual-gain PHAs & 16 \\
Power & $9 \mathrm{~mW}$ per active PHA ${ }^{*}+30 \mathrm{~mW}$ per chip \\
Dynamic range & 10,000 (full-scale/trigger-threshold) \\
Integral nonlinearity & $<0.05 \%$ of full scale \\
Differential nonlinearity & $<1 \%$ \\
High/Low gain ratio & 20 \\
ADC type & Wilkinson \\
ADC resolution (both gains) & 11 bits, 12 th bit overflow \\
Shaping & Bipolar, $1.9 \mu$ s to peak \\
Preamp feedback capacitance & $5-75 \mathrm{pF}$, programmable in $5-\mathrm{pF}$ steps \\
Radiation tolerance & Total dose to $\sim 15 \mathrm{krad}$, latchup-free to at least $80 \mathrm{MeV} /\left(\mathrm{mg} / \mathrm{cm}^{2}\right)$ \\
Gain temperature coefficient & $<50 \mathrm{ppm} /{ }^{\circ} \mathrm{C}$ \\
Offset temperature coefficient & $<0.1 \mathrm{channel} /{ }^{\circ} \mathrm{C}$ \\
Operating temperature range & -30 to $+50{ }^{\circ} \mathrm{C}$ \\
Threshold programmability & up to $6 \%$ of $\mathrm{F} . \mathrm{S}$. (each gain), 10-bit resolution \\
\hline
\end{tabular}

*Each dual-gain PHA chain can be separately powered on or off

$\sim 15 \mathrm{krad}$. With use of guard banding, the chip has been found to be latch-up free to at least $80 \mathrm{MeV} /\left(\mathrm{mg} / \mathrm{cm}^{2}\right)$.

Table 5 summarizes key characteristics of the PHASIC chip. In the STEREO application, for a $20-\mu \mathrm{m}$ thick detector with $440-\mathrm{pF}$ capacitance, the estimated noise level is $30 \mathrm{keV} \mathrm{rms}$. A photo of the PHASIC hybrid is shown in Fig. 25.

\subsubsection{The Minimal Instruction Set Computer (MISC)}

The microprocessor used in LET and SEP Central is the P24 MISC (Minimal Instruction Set Computer), designed at Caltech with the aid of Dr. C. H. Ting. The design derives from earlier MISC implementations developed by Chuck Moore (MuP21; see http:// www.ultratechnology.com/mup21.html) and C. H. Ting (P8 and P16) and is simple enough to fit within a field-programmable gate array (FPGA), yet powerful enough to provide the needed onboard event analysis capability. The microprocessor used in HET and SIT is called the CPU24 and is based on the P24, implemented with some modifications at GSFC to tailor the processor to their applications. Both the P24 and CPU24 designs are implemented in the ACTEL 54SX72-S FPGA, which provides radiation tolerance with its triple-voting architecture.

The MISC employs a RISC-like instruction set with four 6-bit instructions packed into a 24-bit word. Instructions are executed consecutively after a word is fetched from memory. The most significant bit of each instruction designates an I/O buss operation when set. For I/O buss instructions the second most significant bit specifies a write when set, and a read when cleared, while the remaining four bits specify the I/O buss address. For non-I/O buss instructions the most significant bit is cleared and the remaining 5 bits specify 32 possible instructions, 31 of which are implemented.

Following is a list of distinctive features of the P24:

24-bit address and data busses

6-bit RISC-like CPU instructions 
4-deep instruction cache

24-deep data stack

24-deep return stack

Uses about $75 \%$ of 54 SX72A FPGA registers and logic modules

Current implementation runs at $6.4 \mathrm{MHz}$, using $<0.25 \mathrm{~W}$.

The MISC has the following registers, all 24 bits wide:

A Address Register, supplying address for memory read and write

I Instruction Latch, holding instructions to be executed

$\mathrm{P}$ Program Counter, pointing to the next program word in memory

R Top of Return Stack

$S$ Top of Data stack

$\mathrm{T}$ Accumulator for ALU.

The return stack is used to preserve return addresses on subroutine calls. The data stack is used to pass parameters among the nested subroutine calls. With these two stacks in the CPU hardware, the MISC is optimized to support the Forth programming language.

\subsection{Onboard Processing}

\subsubsection{Coincidence Logic}

Whenever any detector in the LET instrument is triggered, the coincidence logic decides whether a valid event has occurred. The normal requirement for an event is a coincidence between L1A (logical OR of the 15 L1A segments) and L2A (logical OR of the 10 L2A segments), which gives an A-side event, or "Aevent", or between L1B and L2B (giving a B-side event, or "Bevent"). However, the requirements for generating an Aevent or a Bevent signal are programmable-either the L1 or L2 requirement can be omitted, or an L3 requirement can be included (see Fig. 26). These options might prove useful if a detector were to fail.

Given an Aevent or a Bevent signal, several other requirements must be met before a valid coincidence signal is generated. These other requirements are also programmable. For instance, if the event is an internally generated calibration or livetime pulse (a STIM event [see Sect. 4.1], in which case stimtag = TRUE), and reqstim is set to TRUE, then only STIM events will generate a valid coincidence. An event is tagged as a "hazard" event if a new trigger occurs $<N \mu$ s after the coincidence logic is able to accept new events, where $N$ is a commandable value (presently $2.8 \mu \mathrm{s}$ ). Table 6 lists the state of the programmable inputs to the coincidence logic after LET is booted.

Once the requirements for a valid coincidence have been met, the event data are read out into a buffer and queued for subsequent processing by the LET onboard event processing software. The raw event data include six tag bits that indicate which of the six layers of the LET instrument were triggered. These tag bits are used to sort each event into one of 10 classes (see Appendix 2 for details). The number of events in each class is telemetered each minute (coincidence rates).

\subsubsection{Nominal Detector Thresholds}

The minimum energy deposit required to trigger a given high-gain PHASIC channel, commonly referred to as the "threshold", is adjustable by command over a range that extends up to $6.3 \%$ of the high-gain full-scale energy, with a resolution of $\sim 0.1 \%$ of the maximum threshold setting. In order to maximize the dynamic range of a PHA and to maximize the 

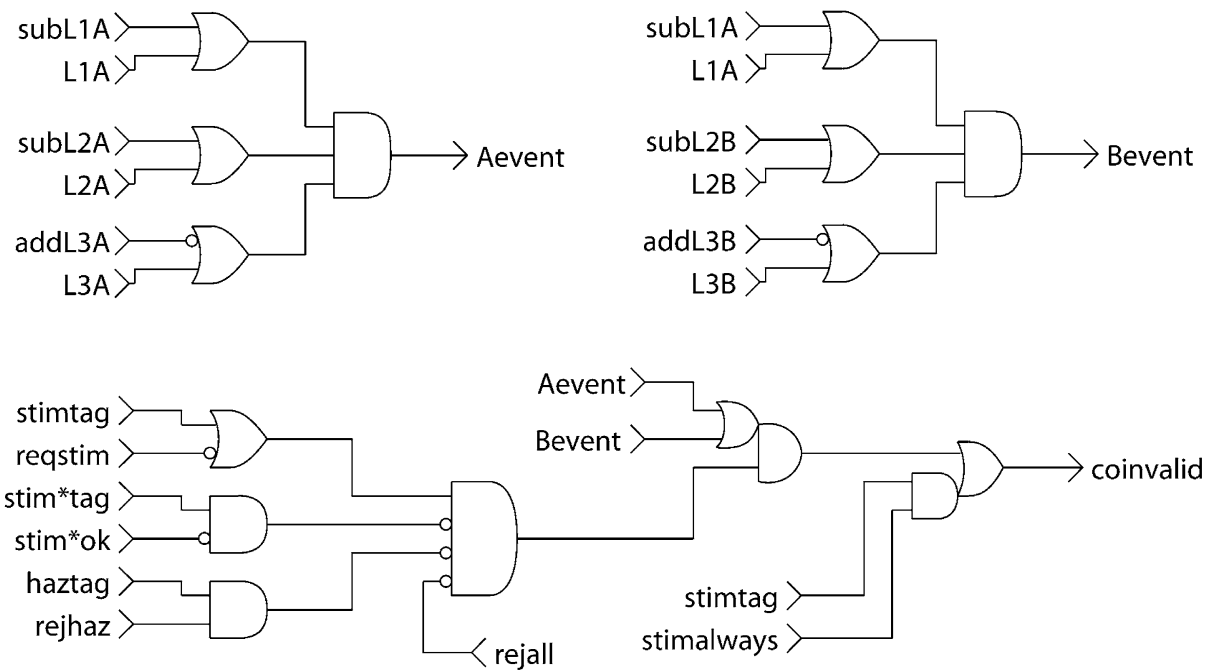

Fig. 26 A diagram of the LET Coincidence Logic. In the above diagram Aevent is a signal (from the front-end logic) indicating that the event is an A-side event, Bevent is a signal indicating that the event is a B-side event, stimtag is a signal indicating that the event is a STIM event, $\operatorname{stim} * \operatorname{tag}$ is a signal that the event is an anti-STIM event (i.e., generated by the negative-going edge of the STIM pulse), haztag is a signal indicating that the event is a hazard event. For other items, refer to Table 6 in Sect. 3.6.1

Table 6 LET coincidence logic programmable inputs

\begin{tabular}{lll}
\hline Input & $\begin{array}{l}\text { Nominal } \\
\text { requirement }\end{array}$ & Description (effect when true) \\
\hline subL1A & FALSE & Delete L1A requirement for Aevent \\
subL2A & FALSE & Delete L2A requirement for Aevent \\
addL3A & FALSE & Include L3A requirement for Aevent \\
subL1B & FALSE & Delete L1B requirement for Bevent \\
subL2B & FALSE & Delete L2B requirement for Bevent \\
addL3B & FALSE & Include L3B requirement for Bevent \\
reqstim & FALSE & Require STIM event for valid coincidence \\
stim*ok & FALSE & Allow anti-STIM events ${ }^{+}$to generate valid coincidence \\
rejhaz & TRUE & Reject events with hazard tags \\
rejall & FALSE & Reject all events. However, STIM events will still be \\
& TRUE & accepted if stimalways is true. \\
stimalways & Always accept STIM events \\
\hline
\end{tabular}

${ }^{+}$An anti-STIM event is one generated by the negative-going edge of the STIM pulse

energy range over which protons can trigger a coincidence, it is desirable to set the high-gain L1 and L2 thresholds as low as possible (without allowing them to trigger excessively on noise). There is also a second consideration-it is important to avoid excessive triggering of L1 and L2 by electrons in order to minimize deadtime due to these triggers, and to avoid electron triggers that might lead to chance coincidences with other single-detector triggers. 
Table 7 Nominal low- and high-gain thresholds and full-scale energies

\begin{tabular}{llll}
\hline Detector & $\begin{array}{l}\text { Nominal high-gain } \\
\text { threshold }(\mathrm{MeV})\end{array}$ & $\begin{array}{l}\text { Nominal low-gain } \\
\text { threshold }(\mathrm{MeV})\end{array}$ & $\begin{array}{l}\text { Nominal low-gain } \\
\text { full scale }(\mathrm{MeV})\end{array}$ \\
\hline L1 & $\begin{array}{l}0.174(\mathrm{~L} 1 \text { centers) } \\
0.205(\mathrm{~L} 1 \text { edges) } \\
\text { (depending on thickness) }\end{array}$ & 5.4 & 920 \\
L2 & 0.29 & 7 & 1,534 \\
L3 & 1.3 & 18 & 3,989 \\
\hline
\end{tabular}

For the L1 devices, laboratory measurements showed that the noise levels were generally lower in the center regions than in the edge regions, as expected from their lower capacitance (see Fig. 20b). In order to measure protons to as high an energy as possible with uniform directional response, the prelaunch thresholds of the ten $\mathrm{L} 1$ centers in each instrument were adjusted to a constant ratio of $7.05 \mathrm{keV} / \mu \mathrm{m}(\mathrm{Si})$, corresponding to the $\mathrm{d} E / \mathrm{d} x$ of a $12-\mathrm{MeV}$ proton (Janni 1966). The thresholds of the $20 \mathrm{~L} 1$ edge segments were adjusted to achieve a constant ratio of $8.3 \mathrm{keV} / \mu \mathrm{m}(\mathrm{Si})$, corresponding to the $\mathrm{d} E / \mathrm{d} x$ of a $\sim 10 \mathrm{MeV}$ proton. Note that it is reasonable to set the thresholds of the L1 centers somewhat lower because during large SEP events proton measurements will be restricted to these segments once the "dynamic threshold" system raises the thresholds on the L1 edge segments (see Sect. 3.6.3). Following launch, when noise levels were found to be lower, it was possible to lower these thresholds and extend the proton energy coverage such that the mean $\mathrm{keV} / \mu \mathrm{m}$ value for the $\mathrm{L} 1$ centers corresponds to the $\mathrm{d} E / \mathrm{d} x$ of a $14.8 \mathrm{MeV}$ proton, while the mean for the L1 edges corresponds to the $\mathrm{d} E / \mathrm{d} x$ of a $12.3 \mathrm{MeV}$ proton.

The L2 signals are less affected by noise than are the L1 devices. Following launch the L2 thresholds were all adjusted to $0.290 \mathrm{keV}$, corresponding to the $\mathrm{d} E / \mathrm{d} x$ of a $\sim 16.5 \mathrm{MeV}$ proton. The prelaunch L3 thresholds were set to an average level of $1.7 \mathrm{MeV}$. Following launch they were lowered to $1.3 \mathrm{MeV}$. The nominal threshold settings in place in early 2007 are summarized in Table 7, along with the nominal low-gain full-scale energy values.

The electron sensitivity of the L1 and L2 devices was estimated using GEANT and an electron spectrum consistent with that measured in the October 28, 2003 SEP event (Mewaldt et al. 2005b). In addition, an exposure was made to a ${ }^{90} \mathrm{Sr}$ beta source. We expect that $0.5 \%$ of SEP electrons with $>200 \mathrm{keV}$ will trigger L1 and that $<0.03 \%$ with $>300 \mathrm{keV}$ will trigger L2. According to the simulations, less than $4 \times 10^{-5}$ of $>500 \mathrm{keV}$ electrons will trigger a coincidence. In flight the electron sensitivity of the L1 and L2 devices will be calibrated based on electron and proton spectra from the SEPT sensor (Müller-Mellin et al. 2007) during the onset of SEP events. If desired, the thresholds can be individually adjusted by command. Threshold changes are implemented via a programmable current source that changes the offset of the pulse height passed to the peak detector in a linear and predictable manner. Any threshold adjustments will therefore require updating the offset table used for the onboard analysis (Appendix 4) as well as modifying ground-based code used for the analysis of telemetered pulse-height events. The required offset change is equal to the threshold change, when measured in energy units.

It is planned that with these settings LET proton measurements will be obtained to high enough energy to overlap with the HET instrument (von Rosenvinge et al. 2007). The stated requirement for measuring protons in LET is 1.8 to $3 \mathrm{MeV}$ (Sect. 3.1). The improvement in the maximum proton energy has been possible because of the better-than-expected noise 
performance of the PHASIC chip and the fact that the L1 detectors are somewhat thicker than originally planned.

\subsubsection{Dynamic Thresholds}

During large SEP events the single-detector count rates can increase by a factor of as much as $10^{4}$ due mostly to low-energy protons. These elevated single-detector count rates create instrument deadtime and also lead to chance coincidence events involving two separate particles. In order to minimize these effects the LET design includes "dynamic thresholds" in which the trigger threshold on selected PHAs are increased during periods when the count rates are high. This action reduces the count rates of selected detectors, minimizing deadtime and effectively reducing the geometry factor for $\mathrm{H}$ and $\mathrm{He}$ events with minimal effect on the geometry factor for heavy ions with $Z \geq 6$.

The dynamic thresholds are implemented in a three-stage process that is controlled by the summed count rates of those selected detectors that do not participate, as summarized in Table 8 and Fig. 27. In the first stage, the high-gain ADCs on all 20 of the L1 outer segments are disabled. The effective threshold for triggering these devices is thereby raised from $\sim 0.25 \mathrm{MeV}$ to the low-gain thresholds, nominally set at $5 \mathrm{MeV}$ (see Table 7). The result is that neither $\mathrm{H}$ nor $\mathrm{He}$ ions can trigger these higher thresholds except for particles incident at very wide angles (see Fig. 28). As a result, the geometry factor for $\mathrm{H}$ and $\mathrm{He}$ is reduced by a factor of 5 .

In the second stage the high-gain ADCs are disabled on all but the center L1 centers (L1A2 and L1B2), providing a decrease in the effective geometry factor for $\mathrm{H}$ and $\mathrm{He}$ by a second factor of $\sim 5$. At this point LET has reduced angular coverage for $\mathrm{H}$ and $\mathrm{He}$ ( $\sim 90^{\circ}$ coverage instead of $\sim 130^{\circ}$ ), but the angular coverage for $Z \geq 6$ ions is not affected (except at the lowest and highest energies, where the L1 and L2 thresholds have some minor effects).

In the third stage the high-gain thresholds are disabled on all but the center two L2 segments on both the A and B sides (L2A4, L2A5, L2B4, L2B5 remain enabled), as well as the outside L3A and L3B segments. The nominal L2 and L3 low-gain thresholds are $\sim 7 \mathrm{MeV}$ and $\sim 18 \mathrm{MeV}$, respectively, in order to be above the maximum energy loss of all but very wide-angle protons (see Fig. 29). This effectively reduces the geometry factor for $\mathrm{H}$ and $\mathrm{He}$ by an additional factor of $\sim 4.5$.

The monitor count rate for this process is the sum of all singles rates that are not affected by these changes (the centers of L1A2 and L1B2; L2A4, L2A5, L2B4, and L2B5; and the centers of L3A and L3B). These monitor-rate trigger levels may be changed by command;

Table 8 Dynamic threshold trigger levels and geometry factors

\begin{tabular}{lccc}
\hline Affected detectors & $\begin{array}{l}\text { Trigger rate } \\
\text { (counts/s) }\end{array}$ & $\begin{array}{l}\text { Turn-off rate } \\
\text { (counts/s })\end{array}$ & $\begin{array}{l}\text { H and He geometry } \\
\text { factor }\left(\mathrm{cm}^{2} \mathrm{sr}\right)\end{array}$ \\
\hline L1 outsides & 1,000 & 500 & 0.815 \\
L1 centers except & 5,000 & 2,500 & 0.175 \\
L1A2 and L1B2 & & & 0.0392 \\
$\begin{array}{l}\text { All L2s but L2A4, } \\
\text { L2A5, L2B4, L2B5; }\end{array}$ & 25,000 & 12,500 & \\
L3 outsides & & & \\
\hline
\end{tabular}


Fig. 27 Illustration of the effect of dynamic thresholds on the count rates and event readout from LET during a solar event with the composition and spectra of the July 14, 2000 (Bastille Day), SEP event. As the thresholds of various detector segments are gradually raised in response to the Monitor rate, the measured singles rate (labeled "scaled Singles") and the $\mathrm{H}$ and He event rates are reduced, thereby preserving instrument livetime to record a greater sample of $Z \geq 6$ events

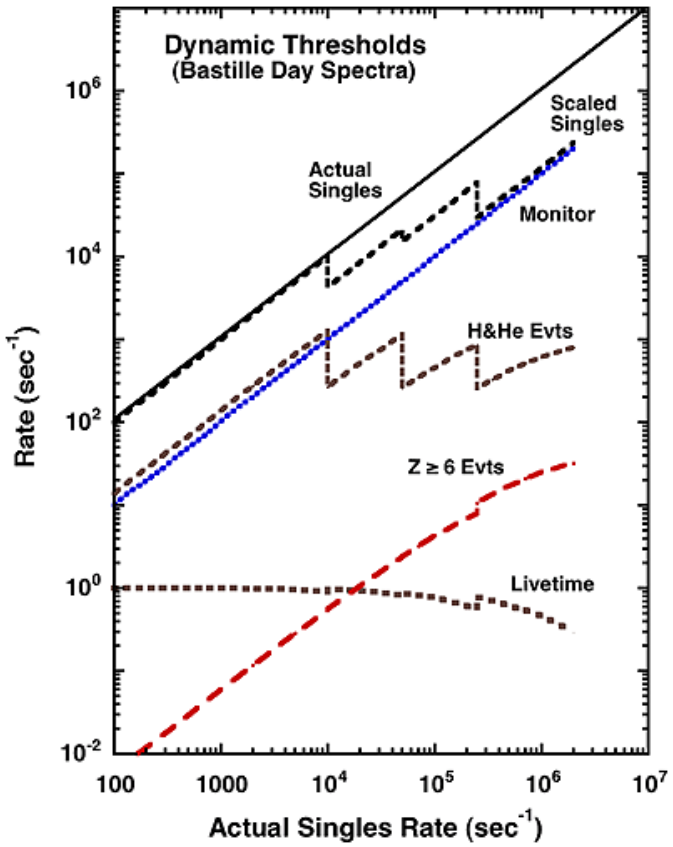

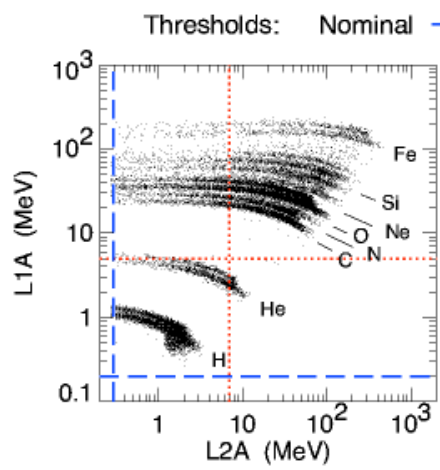

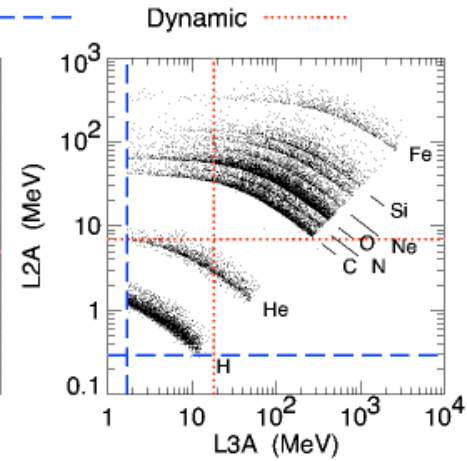

Fig. 28 Illustration of the nominal and dynamic thresholds for L1A•L2A events (left panel) and for $\mathrm{L} 1 \mathrm{~A} \bullet \mathrm{L} 2 \mathrm{~A} \bullet \mathrm{L} 3 \mathrm{~A}$ events (right panel)

the nominal levels are summarized in Table 8. In order to avoid toggling back and forth when the rates are near the trigger levels the dynamic thresholds for a given stage do not return to their nominal level until the count rates drop below the trigger level by some (commandable) factor (nominally $=2$; see Turn-off Rate in Table 8). By disabling the high-gain thresholds, the singles rates in large events such as July 14, 2000 (Bastille Day event) and October 28, 2003 will be reduced by a factor of $\sim 10$, with a corresponding increase in livetime (see Fig. 27). The state of the dynamic threshold currently implemented is indicated by two of the "miscellaneous bits" (see Table 20, Appendix 3) in the LET Science Data Frame, allowing the variable thresholds to be properly accounted for in calculating the true particle rates. 
Fig. 29 The Range 3 (L2 vs. L3, or L2L3) LET matrix.

Foreground species are $\mathrm{H},{ }^{3} \mathrm{He}$,

${ }^{4} \mathrm{He}, \mathrm{C}, \mathrm{N}, \mathrm{O}, \mathrm{Ne}, \mathrm{Na}, \mathrm{Mg}, \mathrm{Al}, \mathrm{Si}$, $\mathrm{S}, \mathrm{Ar}, \mathrm{Ca}, \mathrm{Fe}$, and $\mathrm{Ni}$, and these are shown in alternating green and yellow bands. Background regions are blue, and live STIM boxes are shown in orange. The data points are from the December 13, 2006, SEP event. Note that only 1 in 100 of the events below $\mathrm{C}$ have been plotted

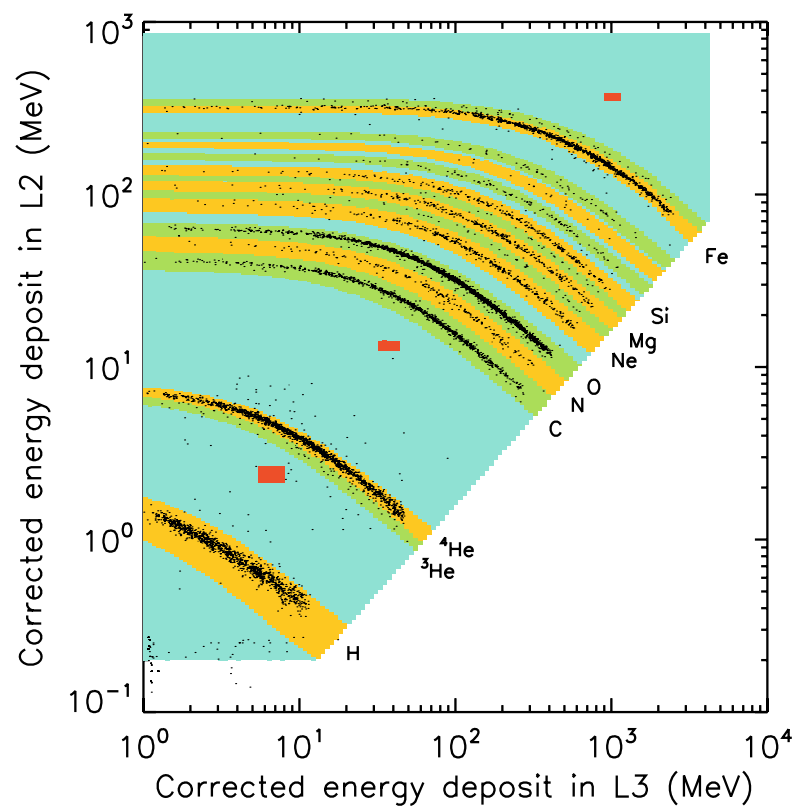

\subsubsection{Onboard Particle Identification System}

The telemetry bandwidth allocated to LET (576.5 bps) is adequate to telemeter only a fraction of the events recorded by the sensor. The raw data for $\sim 4$ events/second can be telemetered, while the onboard particle identification system processes up to $\sim 1,000$ events/second. The objective of the onboard event processing software is to analyze the data gathered for each event and assign a species and energy to the particle that generated the event. For certain species-energy combinations, direction of incidence information is also calculated onboard. In addition, the Range is calculated. Range 2 events trigger only an L1 and an L2 on the same side (but not the L3 on that side). Range-3 events trigger an L1, and L2, and L3 on the same side (but not the other L3), and Range 4 events trigger both L3A and L3B (see Table 19).

ADC-calibration events (see Sect. 4.1) are buffered for telemetry but are not assigned a species or energy onboard. For each non-ADC-cal event acquired by the LET sensor, the onboard software performs the following operations:

1. Sort the event into one of 10 classes based on the tag bits in the event data (see Sect. 3.6.1 and Appendix 2). Increment the appropriate coincidence rate counter based on the classification, and decide whether the event is valid for further processing. An event having one or more of the following properties is not valid:

a. Classification of ERROR or 2TEL based on the tag bits.

b. Events that have multiple hits above a certain threshold in a single detector layer of the instrument.

c. Events for which the particle trajectory results in an ambiguity in deciding whether the particle stops in the instrument or escapes out the side.

d. Events with pulse-height data from an invalid combination of detector layers (the trajectory is not a straight line).

2. For valid Range 2, 3, or 4 events: 


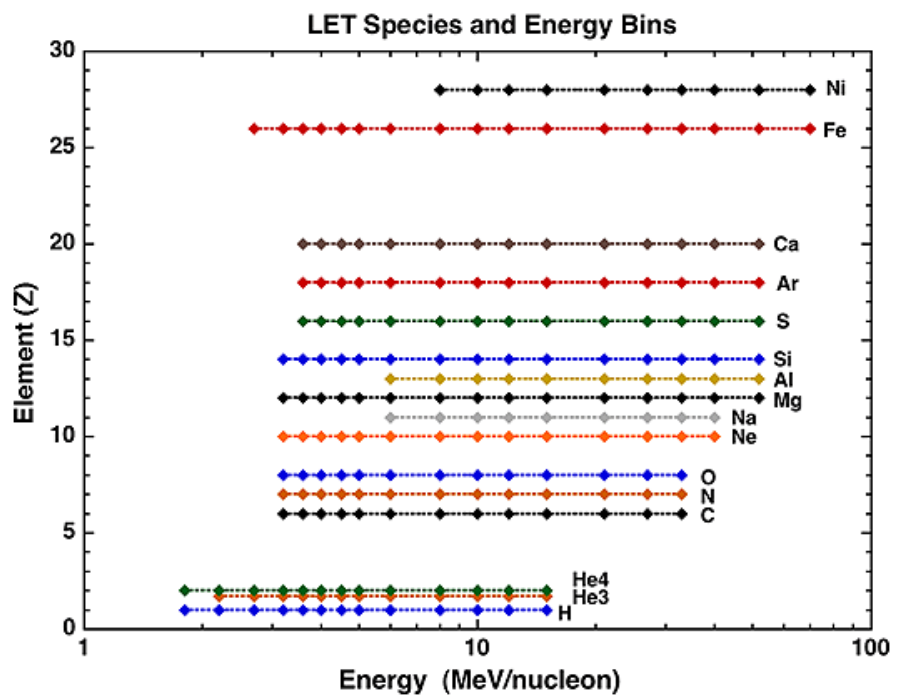

Fig. 30 LET species and energy bins identified in onboard processing

a. Perform gain, detector thickness, and incident angle corrections on the raw pulseheight data from each detector layer.

b. Determine the species (proton, ${ }^{3} \mathrm{He},{ }^{4} \mathrm{He}, \mathrm{C}$, etc.) of the particle by mapping corrected $\Delta E$ and $E^{\prime}$ signals into a two-dimensional detector response matrix. The matrix for Range 3 events is shown in Fig. 29.

c. Determine the total energy of the particle by summing the energy signals from each detector layer and correcting for energy loss in the entrance window.

d. For Range 3 events, treat the particle as an invalid event if the energy in the L2 layer is less than the energy in the L1 layer.

e. For Range 3 and 4 events, treat the particle as an invalid event if the L1/L2 energy ratio is below a commandable threshold.

f. Map the particle into a species-energy/nucleon bin using the calculated species and total energy, and increment the appropriate rate counter. The species and energy bins into which the onboard software maps particles are shown in Figs. 30 and 31.

g. For selected species/energy ranges, determine look-direction (direction of incidence) using the Range 1 and Range 2 detector-combination for the event, and increment the appropriate sectored rate counter (see Sect. 4.7).

3. For invalid events: Sort the event into one of four bins: Range2-Low $Z$, Range2-High $Z$, Range3/4-Low $Z$, Range3/4-High $Z$. The largest signals in each layer are compared with software threshold levels in a simple algorithm to perform this sort.

4. Prioritize all events for telemetry using the priority scheme described in Sect. 3.6.5.

Some of the operations performed onboard deserve more detailed description:

Detector thickness and incident angle corrections: Section 3.3 describes how the $\Delta E$ and $E^{\prime}$ signals can be scaled so that they lie on the track corresponding to the nominal detector thickness, $L_{0}$, and $\theta=\theta_{0}$. In the onboard processing, lookup tables are used to provide the appropriate correction factors for each range and $\Delta E-E^{\prime}$ detector combination.

Treatment of events with multiple hits in a single detector layer: A large signal in one channel of a PHASIC can cause small cross-talk signals in other channels on the same 


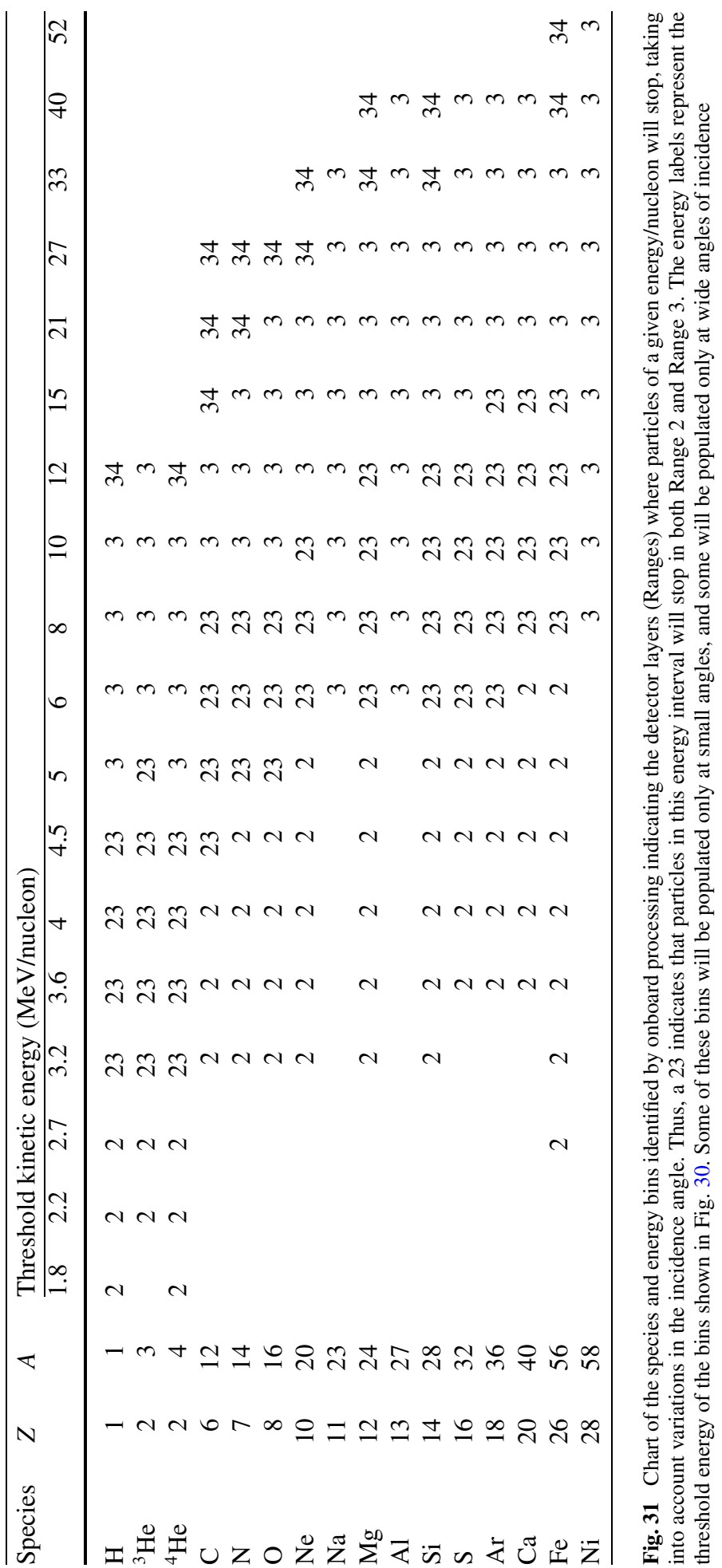


PHASIC. Bench and accelerator calibration tests show that these cross-talk signals never exceed an amplitude of $~ 20$ ADC channels, and that they are not created unless the "real" signal is greater than 750 ADC channels. An algorithm has been implemented in the onboard processing that attempts to identify cross-talk signals in events with multiple hits in a single detector layer. If, after discarding hits that appear to be the result of cross-talk, the event no longer has multiple hits, it is treated as a valid event.

There are a large number of programmable variables and tables used to control features of the onboard real time particle identification system. ADC offsets and gains, thickness and angle correction factors, allowed detector combinations, and other variables can all be changed by command. Various consistency cuts applied to each event can also be enabled/disabled or adjusted, as can the criteria used to identify signals arising from ADC cross-talk. These programmable items are described in Sect. 3.8.

\subsubsection{The Priority System}

The LET telemetry allocation is normally able to transmit about four complete PHA events/sec in addition to the count rate and housekeeping data (see Sect. 5.3). While this is expected to be adequate to transmit essentially all interesting events during quiet times, it will not be possible to keep up with the $Z \geq 6$ events, let alone the $\mathrm{H}$ and He event rate, during SEP events in which the intensity of $Z \geq 6$ ions with $E>3 \mathrm{MeV} /$ nucleon is more than $\sim 1 / \mathrm{cm}^{2}$ sr-sec. However, it is desirable to transmit as many $Z \geq 6$ events as possible at these times in order to obtain statistically accurate measurements of the abundance of elements not covered by the onboard particle identification system. In addition, it is especially important at these times to telemeter a broad selection of events in order to evaluate the performance of the instrument in an extreme environment. The LET priority system is designed to select a comprehensive sample of events for transmission while at the same time giving preference to those categories of events that are most interesting scientifically.

There are a total of 29 priority buffers, of which 27 are currently in use (see Table 9). Each of these buffers can hold up to eight events at a time. Events identified as belonging to an already full buffer are dropped after being counted. The events to be read out are selected by a "round-robin" system from a list of 240 entries. The "weight" assigned to a given buffer indicates the number of times the buffer appears on the list, so that buffers with heavier weights are read out more often. Thus, for example, every third entry on the list is for buffer number 3 (Range 3 ions with $9 \leq Z \leq 30$ ).

During solar quiet periods it will be possible to go through this list many times per minute since most buffers will be empty. During a large SEP event it should still be possible to cycle completely through the list once per minute, since many buffers are rarely populated and, on average, four events/second can be telemetered. Thus all 29 buffers will be read out at least once per minute if there are events available, with the oldest event in a given buffer read out first. Events need not be telemetered during the same minute in which they occurred; if an event's readout is delayed, time bits are set in the event header to provide event latency information (see Latency Tag in Table 22).

The weights were assigned taking into account the scientific priority of the class of events, as well as their expected frequency of occurrence. Thus, since events with $Z \geq 40$ have high scientific priority, they are given five slots even though it is very unlikely that five events will occur in one minute. Range 3 events with $9 \leq Z \leq 30$ are given the greatest weight (occur most often on the list) because they provide the opportunity to measure the spectra and abundances of additional species not covered by the onboard analysis. The assigned weights can be altered by command. 
Table 9 LET priority buffers

\begin{tabular}{|c|c|c|}
\hline Buffer & Weight & Description \\
\hline 0 & 6 & ADC-cal \\
\hline 1 & 5 & Range 2,3 , or 4 with $Z \geq 40$ \\
\hline 2 & 5 & Range 2,3 , or 4 with $31 \leq Z \leq 39$ \\
\hline 3 & 80 & Range 3 with $9 \leq Z \leq 30$ \\
\hline 4 & 40 & Range 2 with $9 \leq Z \leq 30$ \\
\hline 5 & 30 & Range $3 \mathrm{LiBeB}$ and $\mathrm{CNO}$ \\
\hline 6 & 15 & Range $2 \mathrm{LiBeB}$ and $\mathrm{CNO}$ \\
\hline 7 & 8 & Range $4 \mathrm{LiBeB}, \mathrm{CNO}$, and $9 \leq Z \leq 30$ \\
\hline 8 & 2 & Range 4 or "Range 5" penetrating $Z>30$ \\
\hline 9 & 8 & Matrix-sort Reject events with L3 and $Z \geq 3$ \\
\hline 10 & 4 & Matrix-sort Reject events with no L3 and $Z \geq 3$ \\
\hline 11 & 4 & Range $3{ }^{3} \mathrm{He}$ \\
\hline 12 & 3 & Range $2{ }^{3} \mathrm{He}$ \\
\hline 13 & 3 & Range 4 or "Range 5 " penetrating $3 \leq Z \leq 30$ \\
\hline 14 & 5 & Range $3{ }^{4} \mathrm{He}$ and $\mathrm{He}$ background \\
\hline 15 & 1 & Range $4 \mathrm{He}$ \\
\hline 16 & 4 & Range $2{ }^{4} \mathrm{He}$ and He background \\
\hline 17 & 4 & Range $3 \mathrm{H}$ \\
\hline 18 & 1 & Range $4 \mathrm{H}$ \\
\hline 19 & 4 & Range $2 \mathrm{H}$ \\
\hline 20 & 2 & Matrix-sort Reject events with L3, $Z<3$ \\
\hline 21 & 1 & Matrix-sort Reject events with No L3, $Z<3$ \\
\hline 22 & 1 & Range 4 or "Range 5 penetrating" $\mathrm{H}$ and $\mathrm{He}$ \\
\hline 23 & 1 & Range 2,3 , or 4 "Backward" events \\
\hline 24 & 1 & "Clean” Livetime STIM events \\
\hline 25 & 1 & "Poor" Livetime STIM events \\
\hline 26 & 1 & ERROR - Onboard processing of event was aborted due to an error \\
\hline 27 & 6 & Spare \\
\hline 28 & 5 & Spare \\
\hline
\end{tabular}

\subsubsection{Livetime Measurement}

The fraction of the time for which the LET front-end electronics are "alive" and able to respond and read out data for incident particle events is measured using a gated counter in the FPGA logic. The counter is enabled only when the front-end electronics are in the "ready" state, awaiting a trigger. Additionally, the livetime is monitored by the periodic creation of artificial "Livetime STIM" events using the PHASIC built-in test pulsers. These events are designed with specific pulse heights and detector trigger combinations to mimic events of each of the three ranges (Range 2, Range 3, and Range 4) at three different pulse height levels in each range, and are processed entirely through the onboard analysis software in the same way as particle-induced events. The Livetime STIM events with pulse heights falling within their expected boxes in the event matrices (Fig. 29 and Appendix 3) are referred to as "clean", while those falling outside the boxes are "poor" (Table 9). "Poor" Livetime STIM events may appear during high-rate periods such as SEP events when the Livetime STIM 
pulse heights are distorted by chance coincidences with real particle events. The known generation rate of these events allows a cross-check on the gated counter livetime measurement.

\subsection{LET Data}

LET science and housekeeping data are accumulated and transmitted at one-minute intervals. LET is allocated 16 CCSDS packets of science data per minute in telemetry (see Sect. 5.3). The data formatted into these 16 packets constitute a LET Science Frame. The contents of the LET Science Frame are described in Appendix 3. The 11-byte header and 1byte checksum for each 272-byte CCSDS packet are not included in the LET Science Frame format. Therefore, one minute of LET science data corresponds to $16 \times 260=4,160$ bytes, and this defines the length of one LET Science Frame. The LET CCSDS packets are transmitted once every three seconds to SEP Central, with nulled and unused 17th through 20th packets following the 16 Science Data packets. The SEP Central MISC fills in time stamps and checksums in the CCSDS packets before passing them to the IDPU. Due to the 16 CCSDS packet LET telemetry allocation, SEP Central transmits only 16 packets for each LET Science Data Frame and does not transmit the empty 17th through 20th packets. However, the limit is commandable, so that up to 20 packets may be used for data if more bandwidth becomes available to LET later in the mission.

LET generates and sends rate, pulse height, and housekeeping data at intervals of one minute. Data collected during one minute are packaged and transmitted in CCSDS packets over the next minute. Rate and pulse height data are packaged and sent in a LET Science Frame (see Appendix 3). LET housekeeping data are transferred to SEP Central once per minute to be combined with housekeeping data from the other SEP sensors and telemetered in a separate SEP Housekeeping CCSDS packet (see Sect. 5.3). Housekeeping data sent by LET include leakage currents from all detector segments as well as four temperatures measured on the LET electronics board.

\subsubsection{LET Beacon Data}

STEREO Beacon data are to be broadcast continuously for tracking by NASA, NOAA, and other partners around the world. Much like the Real Time Solar Wind (RTSW) system on ACE (Zwickl et al. 1998), these data are meant to provide a snapshot of interplanetary conditions that can be used for forecasting and nowcasting of space weather. All four SEP sensors contribute to the Beacon data, broadcasting particle intensities on a one-minute time scale. The species and energy coverage provided by the four SEP sensors is illustrated in Fig. 32. During SEP events, it will be possible to construct time-intensity profiles and energy spectra using Beacon data for five key species (or species groups), including $\mathrm{H}, \mathrm{He}, \mathrm{CNO}, \mathrm{Fe}$, and electrons over a reasonably wide energy range. In addition, there is anisotropy (front to back) information available for ions and electrons in a range of energy intervals, and limited information on the ${ }^{3} \mathrm{He} /{ }^{4} \mathrm{He}$ ratio. Compared to the ACE RTSW coverage for energetic particles, STEREO Beacon data provide several times as many items, including, in particular, composition data for $\mathrm{He}, \mathrm{CNO}$, and Fe that are not available in ACE RTSW data, as well as much broader energy coverage.

Details of the species and energy coverage provided by LET are summarized in Table 10 [Beacon data from the other SEP instruments are discussed in Müller-Mellin et al. (2007), Mason et al. (2007), and von Rosenvinge et al. (2007)]. Each of the LET items is obtained from the onboard matrices described in Sect. 3.6.4 and Appendix 3. All items but three are summed over all directions of incidence; for one proton and two He intervals the data from 


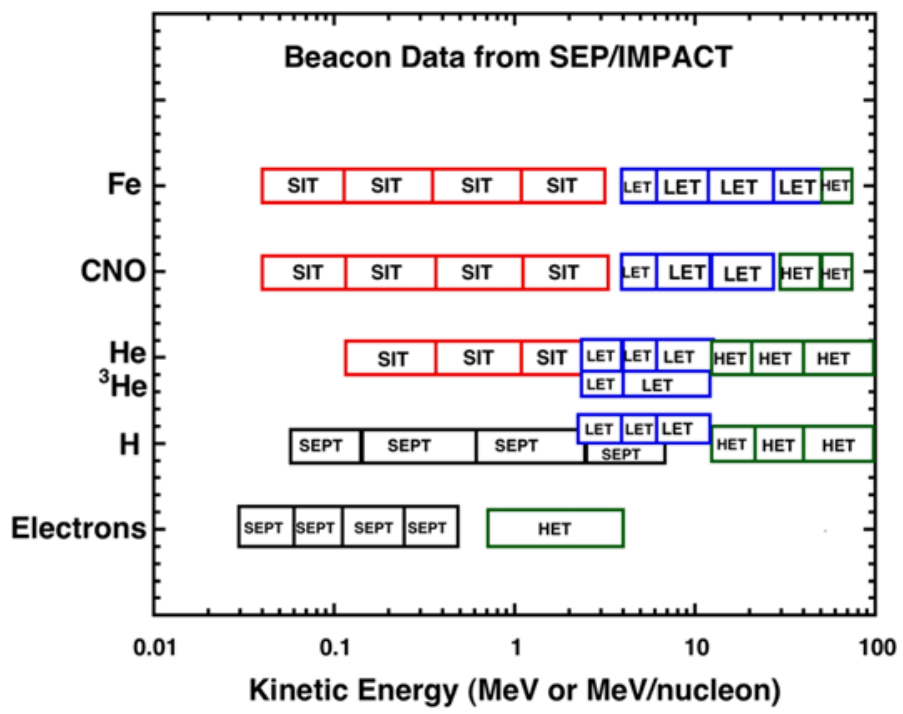

Fig. 32 Species and energy coverage provided in STEREO Beacon data by the four SEP sensors

the A and B sides of LET are separated in order to provide a rough measure of the intensity ratio of particles streaming away from and towards the Sun along the average interplanetary field line. The data from $\mathrm{C}, \mathrm{N}$, and $\mathrm{O}$ are summed in order to improve the statistical accuracy during quiet times and small SEP events.

Also broadcast in the LET Beacon telemetry stream are several items needed to compute absolute intensities, including the instrument livetime, and the trigger, hazard, and accepted event rates (see Appendix 3). The status of the dynamic threshold system is included so that the effect of disabling the high-gain thresholds for some detector segments can be factored into computing the effective geometry factor during any given minute (this only comes into play in large SEP events, as described in Sect. 3.6.3).

\subsection{The Command System}

The command system for the SEP suite of instruments is described in detail in Sect. 5. Briefly, SEP Central manages the command interface between LET and the spacecraft. SEP Central receives LET commands from the ground via the IMPACT IDPU as CCSDS telecommand packets (CCSDS 2000). SEP Central unpacks the commands and routes them to LET via a bidirectional serial command interface. Command responses from LET are routed back to the spacecraft via the same path. This section provides a summary of the key commandable functions and parameters in LET

There are a wide range of parameters and functions for the operation of the LET instrument that can be modified by command in order to optimize its performance and to preserve as many as possible of the instrument functions in the event of in-flight component failures or degradation. Table 11 summarizes these commandable functions. In addition, there are a wide variety of commandable parameters that control ADC thresholds, the ADC and livetime STIM pulsers, and in-flight calibration. It is also possible to modify the priority system, the event format, and various software parameters that define the classification and priority of events. 
Table 10 LET beacon data

\begin{tabular}{|c|c|c|c|}
\hline Species or item & $\begin{array}{l}\text { Energy range } \\
\text { (MeV/nucleon) }\end{array}$ & $\begin{array}{l}\text { Geometry factor } \\
\left(\mathrm{cm}^{2} \mathrm{sr}\right)\end{array}$ & Comments \\
\hline Protons & $2.2-4$ & 4.0 & Summed over $258^{\circ}$ \\
\hline Protons, A-side & $4-6$ & 2.0 & A-side $129^{\circ}$ sector \\
\hline Protons, B-side & $4-6$ & 2.0 & B-side $129^{\circ}$ sector \\
\hline Protons & $6-12$ & 4.0 & Summed over $258^{\circ}$ \\
\hline${ }^{4} \mathrm{He}$ & $2.2-4$ & 4.0 & Summed over $258^{\circ}$ \\
\hline${ }^{4} \mathrm{He}, \mathrm{A}-\mathrm{Side}$ & $4-6$ & 2.0 & A-side $129^{\circ}$ sector \\
\hline${ }^{4} \mathrm{He}, \mathrm{B}-\mathrm{Side}$ & $4-6$ & 2.0 & B-side $129^{\circ}$ sector \\
\hline${ }^{4} \mathrm{He}, \mathrm{A}-\mathrm{Side}$ & $6-12$ & 2.0 & A-side $129^{\circ}$ sector \\
\hline${ }^{4} \mathrm{He}, \mathrm{B}-\mathrm{Side}$ & $6-12$ & 2.0 & B-side $129^{\circ}$ sector \\
\hline${ }^{3} \mathrm{He}$ & $2.2-4$ & 4.0 & Summed over $258^{\circ}$ \\
\hline${ }^{3} \mathrm{He}$ & $4-12$ & 4.0 & Summed over $258^{\circ}$ \\
\hline $\mathrm{CNO}$ & $4-6$ & 4.0 & Summed over $258^{\circ}$ \\
\hline $\mathrm{CNO}$ & $6-12$ & 4.0 & Summed over $258^{\circ}$ \\
\hline $\mathrm{CNO}$ & $12-27$ & 4.0 & Summed over $258^{\circ}$ \\
\hline $\mathrm{Fe}$ & $4-6$ & 4.0 & Summed over $258^{\circ}$ \\
\hline $\mathrm{Fe}$ & $6-12$ & 4.0 & Summed over $258^{\circ}$ \\
\hline $\mathrm{Fe}$ & $12-27$ & 4.0 & Summed over $258^{\circ}$ \\
\hline $\mathrm{Fe}$ & $27-52$ & 4.0 & Summed over $258^{\circ}$ \\
\hline Livetime counter & & & Scaled from 24 to 16 bits \\
\hline Trigger rate & & & 16-bit compressed \\
\hline Hazard rate & & & 16-bit compressed \\
\hline Accepted event rate & & & 16-bit compressed \\
\hline Mode bits & & & $\begin{array}{l}\text { Dynamic threshold state; } \\
\text { Internal code check; } \\
\text { Heater duty cycle; } \\
\text { state of leakage } \\
\text { current balancing. }\end{array}$ \\
\hline
\end{tabular}

Other LET commands are possible, but would first need to be qualified on the LET EM unit prior to execution on any flight unit. Commands related to boot-up, HV control, etc. are really SEP Central commands, and are described Sect. 5. A list of variables and tables that control features of the LET onboard real-time particle identification system is given in Appendix 4. The contents of these variables and tables are commandable.

\subsection{Mechanical and Thermal Design}

\subsubsection{Mechanical Design}

The mechanical design requirements for SEP included:

- provide clear instrument fields of view along the Parker spiral

- withstand vibration and acoustic environments at launch

- equalize internal and external pressures during launch phase

- provide a purge flow of dry nitrogen to protect detectors from volatile contaminants prior to launch 
Table 11 LET command summary

\begin{tabular}{|c|c|}
\hline Item & Comments \\
\hline \multicolumn{2}{|l|}{ Instrument operation } \\
\hline ADC thresholds & Adjust trigger thresholds for all ADCs \\
\hline Coincidence logic & See Table 6 and Appendix 2 \\
\hline ADC STIM pulsers & Change pulse-rate, amplitudes \\
\hline Livetime STIM pulsers & Change pulse-rate, amplitudes \\
\hline Operational heaters & Change heater threshold settings \\
\hline Dynamic threshold states & $\begin{array}{l}\text { Enable/disable, plus the specifications of each state are } \\
\text { programmable in detail }\end{array}$ \\
\hline Data telemetry rate & $\begin{array}{l}\text { The number of LET packets telemetered/minute is } \\
\text { programmable. Extra packets would be filled with events. }\end{array}$ \\
\hline \multicolumn{2}{|l|}{ Software parameters } \\
\hline ADC offsets & Set low- and high-gain ADC offsets (ADC channels) ${ }^{*}$ \\
\hline ADC gain corrections & Set low- and high-gain ADC gain corrections ${ }^{*}$ \\
\hline Detector thickness corrections & Set detector thickness corrections ${ }^{*}$ \\
\hline L1 window corrections & Set L1 detector window thickness corrections ${ }^{*}$ \\
\hline Incident angle corrections & $\begin{array}{l}\text { Set incident angle corrections for each L1-L2 detector } \\
\text { combination }\end{array}$ \\
\hline $\begin{array}{l}\text { Included detector } \\
\text { combinations }\end{array}$ & $\begin{array}{l}\text { Enable/disable each L1-L2 detector combination for } \\
\text { inclusion in onboard processing }\end{array}$ \\
\hline Look-direction sectors & Define L1-L2 detector combinations for each look-direction \\
\hline Priority buffer slots & $\begin{array}{l}\text { Define number of telemetry slots for each event priority } \\
\text { buffer }\end{array}$ \\
\hline Event categories & $\begin{array}{l}\text { Define event categories based on combination of LET } \\
\text { detector layers }\end{array}$ \\
\hline \multirow[t]{2}{*}{ Telemetered event format } & Enable/disable telemetry of cross-talk ADC signals \\
\hline & Set max number of ADCs telemetered/event \\
\hline $\begin{array}{l}\text { Particle ID consistency } \\
\text { cuts }\end{array}$ & Enable/disable consistency cuts for Range 3 and 4 events \\
\hline Raw event integrity & Enable/disable onboard checking/fixing of event headers \\
\hline Hi- $Z /$ Lo- $Z$ cuts & Set $\Delta E$ and $E^{\prime}$ levels for prioritizing events \\
\hline Crosstalk cuts & Set ADC levels for detection of cross-talk signals \\
\hline
\end{tabular}

*For use in onboard processing only. Settings do not affect telemetered events

- minimize weight

- provide RF shielding and a continuous, grounded electrostatic shield

- prevent light from reaching any of the particle detectors

- make it possible to meet the thermal requirements

- maintain a common design for each spacecraft to the extent possible

The initial proposal for the IMPACT Solar Energetic Particles (SEP) instruments had all the SEP telescopes on each spacecraft mounted together as a single unit. This was in part to meet the weight and power constraints. As the spacecraft design evolved, it became apparent that it was not possible to simultaneously meet the field of view constraints of each of the SEP telescopes and to have them mounted as single units on each spacecraft. 

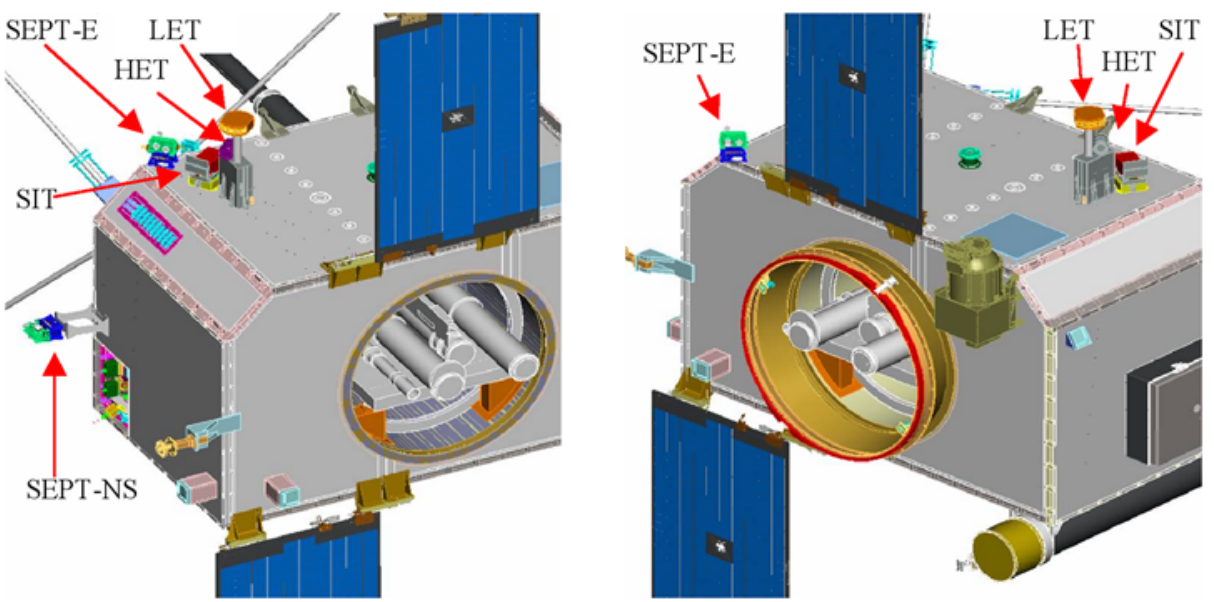

Fig. 33 The IMPACT SEP suite on the body of the Ahead (left) and Behind (right) spacecraft, including SEPT, SIT, LET, and HET. Note that the Behind spacecraft is shown upside down to indicate the placement of the SEP sensors (which are mounted on the panel facing the south ecliptic pole)

By and large, the Ahead and Behind spacecraft designs are identical. However, the orientation of the interplanetary magnetic field (IMF) at $1 \mathrm{AU}$ affects the location of LET and other SEP sensors. The SEP Fields of View (FOVs) are oriented at $45^{\circ}$ to the spacecraft-Sun line so as to look along the nominal Parker spiral direction at $1 \mathrm{AU}$. The Behind spacecraft is predominantly a copy of the Ahead spacecraft rolled $180^{\circ}$ about the spacecraft-Sun line. This points each dish antenna towards the Earth and doesn't disturb the Sun-pointing instruments, but it does mean that the SEP instruments need to be repointed to view along the nominal Parker spiral direction. In order to clear the fields of view with different pointing directions, it was necessary to have different locations for some of the telescopes, including HET and LET, on the two spacecraft. The result is illustrated in Fig. 33. Note that all the SEP instruments on the Ahead spacecraft, with the exception of the SEPT-NS, are on the spacecraft panel that faces the north ecliptic pole, whereas these same instruments on the Behind spacecraft are on the panel facing the south ecliptic pole.

The HET and LET telescopes were kept together with SEP Central, as shown in Fig. 3. Clearing the LET and HET fields of view required raising them fairly high off the spacecraft deck in a direction perpendicular to the launch thrust axis. In particular, LET is mounted on a support tube protruding from the SEP Central enclosure. The support tubes for the Ahead and Behind spacecraft are different, holding the LET telescopes in positions which differ by $90^{\circ}$. Concern about the vibration of this arrangement was allayed initially by NASTRAN analysis of the design and subsequently by actual vibration tests.

Because the LET is located at some distance from SEP Central, the LET electronics are located in the same enclosure that holds the detectors. By contrast, the HET electronics are located inside the SEP Central enclosure. The LET electronics use two multilayer printed circuit boards, one above the detector volume and one below. The board containing the PHASICs, which is less radiation hard than other electronic components, is on the spacecraft-facing side of LET. The two boards are interconnected by a flex-circuit, and the connections between LET and SEP Central are carried by a flex-circuit that passes through the LET support tube. This arrangement minimizes the separation between the PHASIC preamplifier inputs and the detectors. 
RF shielding and light-tightness are maintained by using overlapping double $90^{\circ}$ edges wherever external covers and walls meet. All metal enclosures are iridited on the outside to maintain electrical continuity of the shield. Internal walls are black-anodized in order to promote thermal equilibrium. Vent holes and the natural gaps between mechanical parts provide venting paths to equalize the internal and external pressures during launch. This is required in part to prevent the rupture of thin foils covering the telescope apertures.

Prior to launch, internal Teflon tubing is used to introduce purge gas near the telescope detectors during ground operation, from whence it passes out through the electronics enclosures and escapes through the vent holes. The vent holes point the escaping gas away from the spacecraft in an effort to also carry away any possible dust. The purge gas is dry nitrogen derived from boil-off of liquid nitrogen. It is provided at 15 PSI and is filtered and restricted by sintered metal filters in the purge lines. The LET and HET purge rates are $\sim 5$ and $\sim 1$ liters/hour, respectively. Purging is nearly continuous until just prior to launch.

\subsubsection{Thermal Design}

The thermal design implementations are somewhat different for the LET/SEP Central units on the two STEREO spacecraft, but the design approach is the same. Since the spacecraft does not provide a well-defined thermal environment, the SEP Central enclosure is isolated from the spacecraft using Ultem bushings, which thermally isolate the mounting bolts from the spacecraft. Ground straps were specifically designed by UC Berkeley to provide electrical grounding of the SEP Central enclosure to the spacecraft while maintaining a minimum thermal conductivity.

The minimum and maximum temperatures are expected to occur in two critical situations. For the cold case, this occurs when the solar radiation is a minimum, the instruments are operating with the lowest heat dissipation in the electronic equipment, and the optical properties of the coatings have not been degraded. For the hot case, this occurs when the solar radiation is at the maximum, the instruments are in operational mode with the highest heat dissipation, and the optical properties of the coatings are degraded. The goal of the $\mathrm{SEP} / \mathrm{HET} / \mathrm{LET}$ thermal design is to manage the heat flow in a way that keeps the temperature of the assemblies within the required ranges of $+30^{\circ} \mathrm{C}$ to $-25^{\circ} \mathrm{C}$ operational and $+35^{\circ} \mathrm{C}$ to $-35^{\circ} \mathrm{C}$ nonoperational. The cooling approach includes passive radiators, which radiates to deep space. One important issue was to define the size and position of the radiators required during periods of high heat dissipation in the electronics and high external thermal loads. Conversely, these areas must not be so large that the temperatures would go below the allowed minimum during periods of low heat loads. The radiators are positioned on the side of the SEP Central electronics and LET housing that "see" deep space and consist of Mylar tape covered with a high-emissivity silver-Teflon coating.

Two types of electrical heaters are employed: operational and survival heaters. Operational heater power is enabled when the instruments are on and survival power is enabled when the instruments are off. The heaters are chip resistors mounted on the instrument circuit boards. The operational heater chip resistors are mounted on the LET circuit board only, whereas the survival chip resistors consist of two zones, one mounted on the LET circuit board and the other on SEP Central's Analog/Post-Regulator circuit board. The resistors are mounted near the board attachments to the housing so heat is conducted to the aluminum housing. The insides of both the LET and SEP Central electronics are black anodized to enhance radiation heat transfer. Radiation heat transfer is also enhanced with the conformal coating on the boards. Heat is radiated and conducted from the aluminum housing to the additional circuit boards and also to the detectors in LET. 
The HET sensor is conductively coupled to the SEP Central electronics in order to maintain the desired temperature range. The LET operational heater on the Ahead S/C can supply $1.0 \mathrm{~W}(0.75 \mathrm{~W}$ on the Behind $\mathrm{S} / \mathrm{C})$ at $30.5 \mathrm{~V}$ and $75 \%$ duty cycle. The survival heaters in the Ahead LET/SEP Central can supply $4.3 \mathrm{~W}$ (4.75 W on Behind) at $25 \mathrm{~V}$ and $100 \%$ duty cycle. The operational temperature zone is controlled by a microprocessor that receives feedback from a local thermistor. The operational setpoint can be adjusted remotely via ground commands. At launch it was set at $-10^{\circ} \mathrm{C}$ in both LET units. The survival heaters are controlled by the redundant set of mechanical thermostats that close at $-26.1^{\circ} \mathrm{C}$ and open at $-9.4^{\circ} \mathrm{C}$. The predicted flight temperatures for the LET detectors are $-11^{\circ} \mathrm{C}$ to $+27^{\circ} \mathrm{C}$ for the Sunfacing side and $-21{ }^{\circ} \mathrm{C}$ to $+11^{\circ} \mathrm{C}$ for the shaded side.

Inside LET/SEP Central there are eight internally read-out thermistors and two that are read out by the spacecraft for independent temperature verification and as the only temperature indicator when SEP power is turned off.

There was particular concern that the sunward staring LET detectors not get too hot. Two thin foils, each one-third mil thick Mylar, cover each LET aperture. The inner foil is a circular foil immediately adjacent to each L1 detector. The outer foil is a single long and narrow foil on each end of the LET telescope, covering five apertures. The foils seen in Fig. 3 have a high infrared emissivity $(\varepsilon \sim 0.67)$ and low solar absorptivity $(\alpha \sim 0.1)$ coating (the so-called Goddard composite coating) facing away from the detectors and a lowinfrared emissivity coating (double vacuum deposited aluminum with a combined thickness of $\sim 2.1 \mathrm{mg} / \mathrm{cm}^{2}$ ) facing the detectors. The coating facing away from the detectors keeps the foils facing the Sun cool. The low-emissivity coating facing the detectors minimizes radiation heat transfer exchange between the foils and the detectors. In the event that an outer foil breaks the inner foil provides a redundant foil. The two foils in series also help minimize the radiative exchange between the detectors and outer foil. An outer indium tin oxide (ITO) coating, as required to meet the electrostatic cleanliness specification, is present on the outer surface of the outer foil only and only for the end of the telescope that does not see the Sun. This coating was applied on top of the Goddard composite.

All external areas with the exception of the radiators and telescope apertures are covered with multilayer insulation blanket. The total power dissipated by LET/HET/SEP Central is 6.05 watts.

\subsection{Resources}

The measured mass, power, and allocated data rate, and the heater power for LET and SEP Central, are summarized in Table 12. The LET mass numbers include the electronics, detectors, and the housing. In addition, LET is mounted on a tubular bracket that has a mass of $0.465 \mathrm{~kg}$ (see Fig. 3), and the thermal blankets weigh $0.180 \mathrm{~kg}$ (average of spacecraft A and B). The thermal blanket for SEP Central weighs $0.192 \mathrm{~kg}$ (average). Note that the SEP Central housing also encloses the HET electronics board. This resource is book-kept under the HET sensor.

The SEP Central power values include an efficiency of $56 \%$ for the low-voltage power supply (LVPS). The power values are average values. Note that if any sensor draws additional power for whatever reason, the power dissipation by SEP Central increases because of the 56\% LVPS efficiency. The maximum power that SEP Central could draw is $4.842 \mathrm{~W}$, which could occur if five separate LET detectors were short-circuited and if all other sensors are drawing their maximum power levels.

The data rate shown in Table 12 includes Science, Housekeeping, and Beacon data. 
Table 12 Summary of LET and SEP central resources

\begin{tabular}{lll}
\hline Resource & LET & SEP Central \\
\hline Mass (kg) & 0.855 & 2.196 \\
Power (W) (excluding heaters) & 1.18 & 4.111 \\
Data Rate (bps) & 576.5 & 9.1 \\
Op-heater power (W) & 1.0 Ahead, 0.75 Behind & - \\
Survival heater power (W) & 1.3 Ahead, 1.75 Behind & 3.0 Ahead, 2.75 Behind \\
\hline
\end{tabular}

\subsection{Electrical GSE}

Electrical ground support equipment (EGSE) was developed for communication with SEP Central during development and laboratory testing. This EGSE consists of a pair of computers, one running Windows and the other running Linux. The Windows machine controls the operation of an IDPU Simulator, a custom-designed piece of hardware that has interfaces and functionality equivalent to those of the IMPACT flight IDPU (see Luhmann et al. 2007). Both the IDPU simulator and the software that controls it were supplied by UC Berkeley. The Linux system, which uses a TCP/IP interface to the Windows system, provides the capability to send ASCII commands and do binary uploads to SEP Central and the individual SEP instruments. It collects telemetry data from SEP, logs it to disk, and allows communication with remote computers by means of TCP/IP sockets. This latter feature makes it possible to monitor test data and generate commands from remote sites as well as locally. The Linux system also makes a variety of LET data displays including rates, housekeeping parameters in both raw and engineering units, and information from individual events.

For testing SEP when it is installed on the STEREO spacecraft the Linux system communicates directly with the IMPACT GSE, which provides the interface to the flight IDPU. The EGSE is used during early-orbit operations after launch to provide a real-time display of data collected during the STEREO telemetry pass.

\section{Calibrations and Performance}

\subsection{Electronic Calibrations}

Prior to being installed in the instruments, each PHASIC hybrid used in the LET PHA chains was individually tested over a temperature range of $-35^{\circ} \mathrm{C}$ to $+45^{\circ} \mathrm{C}$ in intervals of $20^{\circ} \mathrm{C}$ $\left(-35^{\circ} \mathrm{C},-15^{\circ} \mathrm{C},+5^{\circ} \mathrm{C},+25^{\circ} \mathrm{C}\right.$, and $\left.+45^{\circ} \mathrm{C}\right)$. Within each assembled LET instrument, there are 54 test pulsers, one for each of the 54 electronics channels. These pulsers are driven by two pulser reference voltages, one for all of the L1 detector segments and one for all of the L2 and L3 segments. Each reference voltage is generated by an eight-bit DAC with two gain states that differ by a factor of 110 . All test pulsers may be pulsed independently, or in coincidence, in any combination, although usually preprogrammed patterns of detector combinations are pulsed at a fixed grid of pulse heights.

There are two varieties of pulser-generated events. In order to monitor instrument livetime (see Sect. 3.6.6), "Livetime STIM" events are generated in detector segments L1A2b, L2A4, L3Ai, L1B2b, L2B4, and L3Bi (i.e., those detectors nearest the instrument centerline), simulating Range 2, Range 3, and Range 4 particles at three different pulse height levels each. The pulse height data from these events should fall into the STIM boxes in the 
event matrices (Fig. 29 and Appendix 3). To avoid saturating the telemetry with Livetime STIM events, their pulse heights are assigned to low-priority readout buffers (buffers 24 and 25; see Table 9). It is the rates of these events, not their pulse heights, which are required to check the livetime measurement.

In order to check the linearity of the PHA response and routinely monitor the stability of all ADC gains and offsets and the electronic noise, a second type of pulser-produced event, "ADC-calibration" events, are periodically generated by pulsing at most a single detector in each of the six layers at 32 different pulse height levels which span the entire dynamic range. (The location of the thresholds on each PHA channel can also be measured on command with a more finely-spaced grid of pulses limited to smaller pulse heights.) A and B sides of the LET instrument are pulsed simultaneously for ADC-cal events, each of which contains an L1A and L1B detector. An L2 or L3 detector is pulsed along with an L1 segment on each side for 12 of the $15 \mathrm{~L} 1 \mathrm{~s}$. Note that this pattern of detector hits, unlike those for the Livetime STIM events, does not mimic that expected for an actual particle event (see Appendix 2). In order to be recognized onboard as a legitimate instrument trigger and undergo pulse height analysis, special provisions were made in the coincidence logic for these ADC-cal events (Sect. 3.6.1 and Fig. 26).

In the default normal mode, 3.5 pulser events are generated every second, however, this rate is programmable on command. Normally 9 seconds out of every 10 are devoted to Livetime STIM events, with 1 second out of every 10 used for ADC-calibration events, some of which get read out each minute (buffer 0; Table 9). The detector configuration and pulse height level is changed at most only once per second. Thus, with 15 different detector configurations (each of which includes one L1A and L1B segment, as described earlier) at 32 different levels being pulsed for one second out of every 10, it normally takes 4,800 seconds (or 80 minutes) to cycle through all 54 ADCs over the entire dynamic range. A commandable mode which disables Livetime STIM and generates only ADC-calibration events may be used to assess the health of all ADCs in only eight minutes, which proved useful during preflight environmental testing.

The ADC-cal events have shown that linearity for all channels is better than $\sim 0.2 \%$ even at the largest pulse heights. Furthermore, since the electronic noise of the high-gain L1 channels is dominated by the detector capacitance of the thin ( $\sim 24$ micron) L1 detectors, the RMS width of the pulsed events is well-correlated with the detector thickness, as illustrated in Fig. 34. This provides a useful check on detector integrity after environmental tests such as the acoustics and vibration tests.

\subsection{Accelerator End-to-End Test and Calibration}

An engineering model of LET was tested at the Lawrence Berkeley Laboratory (LBL) 88" Cyclotron in March 2004 using a mixed, "cocktail" beam (McMahan 2005) of heavy ions ranging from ${ }^{10} \mathrm{~B}(Z=5)$ to ${ }^{209} \mathrm{Bi}(Z=83)$. Although the low-beam energy (4.5 MeV/nucleon) limited the range of most species to Range 2 events, the test proved that the new LET electronic designs could resolve heavy ions over a wide range of intensities and that the instrument had sensitivity to ions ranging from $\mathrm{H}$ up to very heavy ions.

A much more thorough accelerator calibration was performed on both LET flight units at the National Superconducting Cyclotron Laboratory (NSCL) at Michigan State University (MSU) in July 2004 using a beam of $140 \mathrm{MeV} /$ nucleon ${ }^{58} \mathrm{Ni}(Z=28)$. A variable-thickness target upstream of the instrument, consisting of a water-filled bellows aligned with the beam and mounted on a translation stage driven remotely by a stepper motor, allowed the ${ }^{58} \mathrm{Ni}$ beam to be stopped at any desired depth within LET. By continuously varying the thickness 


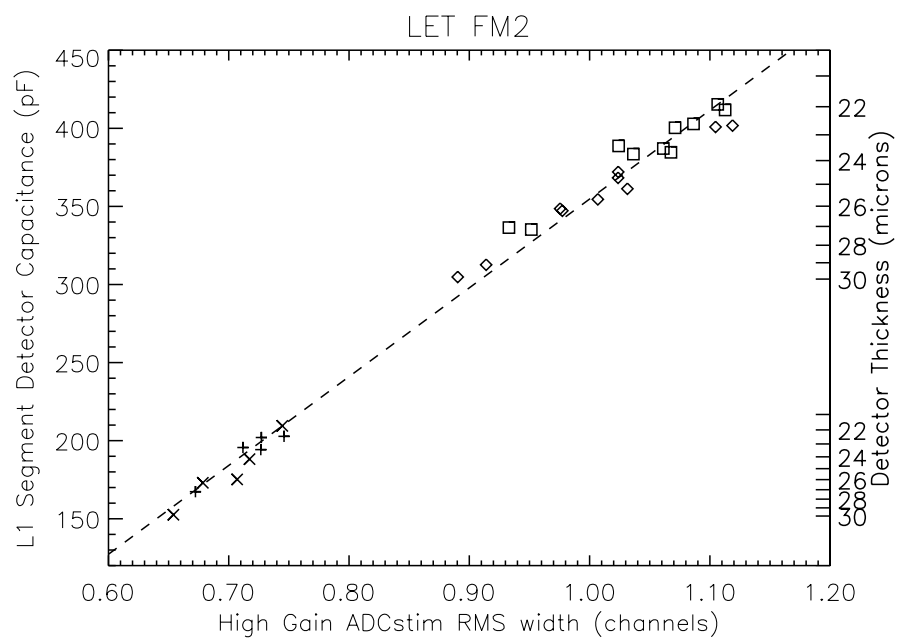

Fig. 34 Calculated L1 segment detector capacitance (based on thicknesses indicated on the right-side axis as determined using alpha particles (see Appendix 1) vs. measured rms width of the high-gain onboard pulser-generated pulseheights. Electronic noise for these thin detectors is dominated by the detector capacitance, which produces the tight correlation and provides a useful tool to check for broken detectors after environmental testing. Crosses and plusses are for circular center segments on sides A and B, respectively, while diamonds and squares are for the larger, semi-annular edge segments on sides A and B

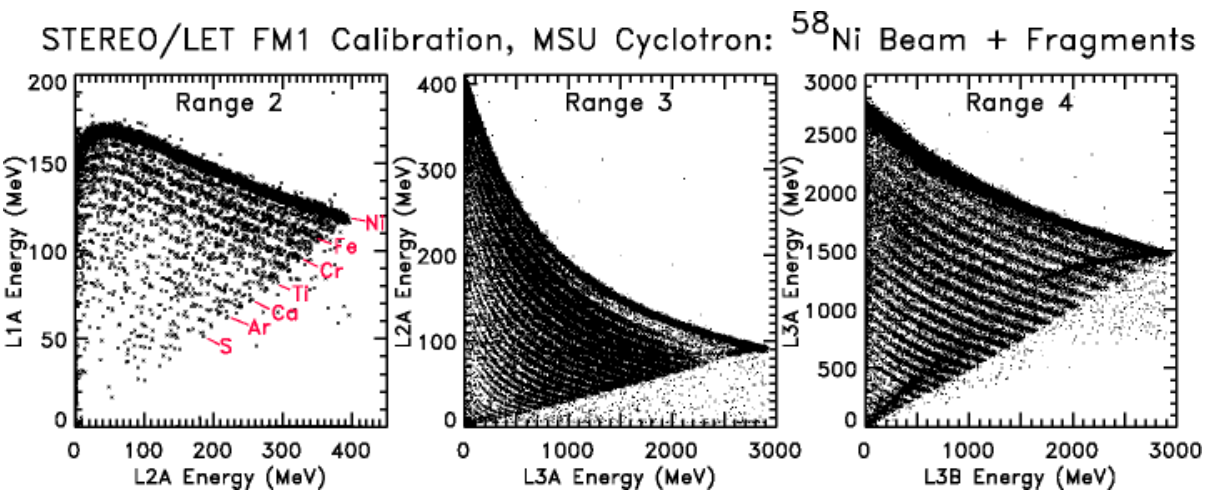

Fig. $35{ }^{58} \mathrm{Ni}$ beam and fragment data from the end-to-end test of LET at the MSU cyclotron. Resolution for $\mathrm{Ni}$ under the conditions shown is approximately $0.18,0.17$, and 0.07 charge units for Range 2, 3, and 4, respectively

of the water target, complete tracks of $\mathrm{Ni}$ at all depths in the instrument were obtained, while by increasing the water thickness in order to stop the Ni beam and serve as a fragmenter, samples of all elements from $\mathrm{H}$ to Ni were produced, as illustrated in Fig. 35. These and similar data were collected with LET mounted in a vacuum chamber (to avoid the otherwise substantial amount of dead material that air would introduce between the L1 and L2 detectors). The entire vacuum chamber was mounted on a rotation stage so the beam could enter LET at a variety of incidence angles, all of which were tilted $5^{\circ}$ off the horizontal to avoid a primary channeling plane of the $\langle 100\rangle$ silicon detectors. 
In addition to mapping the element track locations to better define the onboard matrix boxes, test the accuracy of low-energy heavy-ion range-energy relations, and demonstrate the detector and ADC response with species, energy, and angle, the accelerator tests were used to calibrate and check the performance of a variety of the instrument functions described in Sect. 3.6. These include: (1) the coincidence logic, (2) real-time particle identification, (3) the priority and readout systems, (4) nominal detector thresholds, (5) livetime determination, and (6) dynamic thresholds. Testing some of these features required high beam rates, and a variety of rates from about 0.3 to $18 \mathrm{kHz}$ were employed. Use of a beam of particles of a known species and energy enabled the mapping of segment-to-segment mean thickness variations in the L2 detectors (which were too thick to measure with laboratory alpha sources as was done for the L1 detectors as described in Sect. 3.4), and the relatively large pulse heights of the stopping $\mathrm{Ni}$ ions proved useful for refining onboard algorithms designed to deal with electronic cross-talk (Sect. 3.6.4). As is visible in the third panel of Fig. 35, accelerator testing revealed a pronounced broadening of the Range 4 heavy ion tracks, which has been attributed to the L3 detectors being somewhat less than fully depleted at their nominal flight bias. The decreased resolution is minor enough to not impact the achievement of LET science objectives.

\subsection{Radioactive Source Tests}

Both LET instruments were periodically tested with radioactive sources in order to verify their operation during the integration and test program. Alpha sources $\left({ }^{228} \mathrm{Th}\right.$ and $\left.{ }^{210} \mathrm{Po}\right)$ were used to check the aliveness of the 54 individual detector segments as well as the coincidence circuitry before and after the thermal-vacuum, acoustic, and vibration tests, and following any repairs. In addition, ${ }^{90} \mathrm{Sr}$ beta source was used at times to check the aliveness of all detectors and to verify the response of the L1 and L2 detectors to electrons.

The alpha-particle tests, which were performed at $1 \mathrm{~atm}$. pressure, employed a source holder that mounted five alpha sources (four ${ }^{210} \mathrm{Po}$ and one ${ }^{228} \mathrm{Th}$ ) to either the A or B sides of the LET telescope. A $1-\mu \mathrm{Ci}{ }^{228} \mathrm{Th}$ alpha source was mounted in front of the center $\mathrm{L} 1 \mathrm{de}-$ tector (L1A2 or L1B2) and four ${ }^{210}$ Po sources ( $5 \mathrm{mCi}$ each) were mounted in front of the remaining L1A or L1B detectors. Each source was covered by a thin $(\sim 8.4 \mu \mathrm{m})$ polyethylene foil that was used as a target to produce energetic protons using Rutherford scattering. The ${ }^{210} \mathrm{Po}$ alpha particles did not have sufficient energy to reach the L1 detectors, but the elastically scattered protons produced a count rate of $\sim 5$ to 10 counts/minute in the L1 segments. The maximum alpha-particle energy from the thorium source is $8.785 \mathrm{MeV}$, which yielded protons with energies up to $\sim 5.6 \mathrm{MeV}$. These protons were able to penetrate the L1 detector and reach all of the L2 and L3 segments on a given side, thereby providing functional tests of the detector response, the coincidence circuitry, and the onboard analysis routines. The ${ }^{210} \mathrm{Po}$ and the ${ }^{228} \mathrm{Th}$ half-lives are 138 day and 1.91 year, respectively. The counts rates were corrected for these half-lives to monitor long-term stability.

\subsection{In-Flight Particle Calibrations}

In addition to the electronic calibrations discussed in Sect. 4.1, LET will also be calibrated using in-flight particles. The stability of the detectors and electronics will be monitored by checking whether the elements identified onboard remain centered in the bands in the three matrices as a function of time, temperature, and radiation dose (see Sect. 3.6.4). If not, it is possible to upload revised particle identification matrices. Large SEP events provide excellent statistical accuracy over a short time interval. Since the LET response overlaps 
with those of all three other SEP sensors (see Fig. 1), comparisons of the count rates, composition, and energy spectra between the four sensors will make it possible to continually intercalibrate the SEP suite.

\subsection{Monte Carlo Simulations}

Monte Carlo simulations were used in the design and optimization of the LET instrument to calculate geometry factors, to determine charge and mass resolution, to optimize detector threshold settings, and to test onboard particle identification software. Section 4.6 describes Monte Carlo calculations of the LET geometry factor as a function of particle energy and the various L1, L2, and L3 detector-segment combinations. These calculations also determined the results of modifying the response of the LET detectors dynamically during large SEP events in order to optimize the performance. In these calculations a numeric model of LET was exposed to an isotropic flux of particles with a known composition and energy spectrum.

Numerical simulations of the LET response were also used to determine the charge and mass resolution of the instrument. Although LET was tested with accelerator beams of heavy ions, as discussed in Sect. 4.2, it is impractical with a parallel, monoenergetic beam of a single nuclide to collect data over the full range of positions, angles, energies, and species to which LET will be exposed in space. The simulations for evaluating instrument resolution took into account heavy ion range-energy relations (including the energy dependence of the ions' effective charge) and Bohr/Landau fluctuations. They also used the measured mean thickness of each segment of the individual L1 detectors, to which was added a thickness deviation drawn on a particle-by-particle basis from a Gaussian distribution with a standard deviation equal to the measured rms thickness variation of the segment. The deposited energy in each hit detector segment was calculated and then a noise signal was added based on the typical characteristics of the LET PHASIC and the capacitance of the detector and the associated front-end circuit. Finally these signals were compared with the nominal detector thresholds to determine whether the events would satisfy the LET coincidence requirements and which pulse heights would be included in the telemetry stream. The resulting set of pulse heights were then analyzed using the same algorithms that will be applied to flight data.

Figure 22 shows results obtained from the Monte Carlo calculation using input energy spectra in the form of power laws with exponent -2 . Particles were sampled from an isotropic distribution, but only those incident from the A (sunward) side of the instrument were included in the plots. Particle composition used for this calculation was typical of that found in gradual SEP events with the following exceptions: He and $\mathrm{H}$ have been suppressed relative to the heavier elements by factors of 500 and 5,000, respectively, in order to avoid saturating the plot with these light elements, and ${ }^{3} \mathrm{He}$ has been set to $10 \%$ of ${ }^{4} \mathrm{He}$ in order to illustrate capability for identifying ${ }^{3} \mathrm{He}$ using the onboard analysis. The left-hand panels show the raw pulse heights. For Range 3 (lower left) the element tracks are broadened due to the wide range of angles sampled. For Range 2 (upper right) the angular spread sampled by a given detector is less (because the L1 detectors are arrayed along an arc of a circle) but the range of $\mathrm{L} 1$ detector thicknesses sampled is relatively large. The different $\mathrm{L} 1$ thicknesses cause the multiple tracks for individual elements in this plot. The two plots on the right-hand side of the figure show how the events can be mapped onto well-separated element tracks based on the scaling procedure that is used onboard to approximately correct for spread in angles and $\Delta E$ detector thicknesses, as discussed in Sect. 3.3.

For the subset of events that is telemetered to the ground, one is able to derive particle charge and mass using the full range-energy relation (Stone et al. 1998b; Appendix 1) rather 


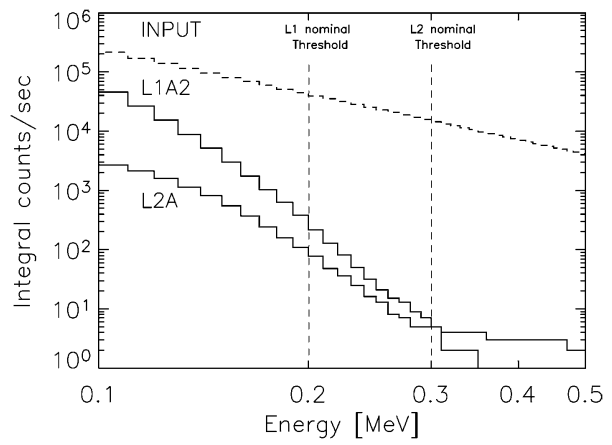

Fig. 36 Monte Carlo simulations of the response of a $25 \mu \mathrm{m}$ thick L1 detector to electrons. The expected integral count rate of electrons hitting an L1A2 detector (top solid line) and penetrating to any L2A detector (lower solid line) is shown as a function of the energy deposit in those detectors. The input spectrum (plotted vs. the incident electron energy) is from the large October 28, 2003, SEP event. The vertical dashed lines indicate the nominal L1 and L2 threshold energies, respectively. Less than $10^{-5}$ of the electrons with $>0.3 \mathrm{MeV}$ will trigger a coincidence between L1 and L2

than the power-law approximation employed onboard. This should yield somewhat better mass resolution, particularly at low energies where the power law approximations are accurate only over relatively narrow energy intervals. To estimate the LET mass resolution Monte Carlo simulations were run in which the input composition contained equal abundance of ${ }^{3} \mathrm{He}$ and ${ }^{4} \mathrm{He}$ or of ${ }^{20} \mathrm{Ne}$ and ${ }^{22} \mathrm{Ne}$. Histograms of the masses calculated for particles stopping in Ranges 2, 3, and 4 were constructed and the mass resolution was obtained from the standard deviations of Gaussian fits to the central portions of the mass peaks. These values are listed in Table 1. The mass resolution in flight should be slightly degraded from that predicted based on the Monte Carlo simulations because of several effects not included in the calculations (statistical fluctuations of the particles' effective charge, multiple Coulomb scattering in the instrument). In some cases resolution can be further optimized by restricting analysis to those combinations of L1 and L2 detector segments for which the uncertainty in the penetrated $\Delta E$ thickness is the smallest.

Simulated events obtained from the Monte Carlo simulations also proved useful for developing and testing the particle identification software implemented in the LET MISC.

Monte Carlo simulations were also made using GEANT-4 to optimize the L1 and L2 $\mathrm{ADC}$ thresholds. If the thresholds are too low, the high electron intensities during a large SEP event may increase the singles rates of L1 and L2 causing additional deadtime, and possibly increasing the chance coincidence rate. Figure 36 shows the expected integral count rates of a single L1 detector and any L2 detector during the October 28, 2003, event (Mewaldt et al. 2005b). Vertical lines indicate the nominal L1 and L2 threshold energies. Note that the ADC thresholds are adjustable by command, as described in Sects. 3.6.2 and 3.8.

\subsection{Energy Range and Collecting Power}

The collecting power and energy range of LET was evaluated for a wide range of key ions using Monte Carlo simulations as described in Sect. 4.5. The energy range spanned by nuclei stopping in Range 2, Range 3, and Range 4 is shown in Fig. 1. Figure 37 shows the result of Monte Carlo simulations of the LET geometrical factor (area solid-angle factor) plotted as a function of particle energy for the abundant isotopes ${ }^{16} \mathrm{O}$ and ${ }^{56} \mathrm{Fe}$. The curves indicate the geometrical factors for particles stopping in Range 2, Range 3, and Range 4, along with 
Fig. 37 LET Geometry Factor vs. energy/nucleon for $\mathrm{O}$ and $\mathrm{Fe}$. The geometry factor for Range 2 , 3 , and 4 events are indicated by dashed, dotted, and dash-dot lines, respectively. The solid line represents the sum of the responses

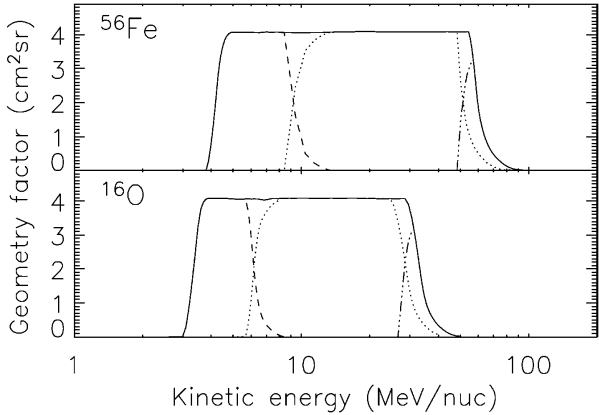

the sum of these responses. The Range 4 response (for the A-side) has been evaluated only to a depth in $\mathrm{L} 3 \mathrm{~B}$ of $0.3 \mathrm{~mm}$ of $\mathrm{Si}$, in order to avoid confusion with heavy particles that completely penetrate both L3A and L3B (see also the Range 4 particle identification matrix in Appendix 3). Over most of the energy range the total geometry factor is $4.0 \mathrm{~cm}^{2} \mathrm{sr}$.

LET will also measure ultraheavy (UH) ions with $Z \geq 30$ that are sometimes overabundant by up to a factor $>1,000$ in impulsive SEP events (Reames and $\mathrm{Ng} 2004$; Mason et al. 2004). During an exposure of the LET engineering unit at the LBL 88-inch cyclotron LET observed UH ions that included ${ }_{65} \mathrm{Cu},{ }_{86} \mathrm{Kr},{ }_{136} \mathrm{Xe}$, and ${ }_{209} \mathrm{Bi}$. The energy range of the $\mathrm{UH}$ response starts at $\sim 4 \mathrm{MeV} /$ nucleon. There are two priority buffers devoted to UH events.

Each of the ten L1 detectors has three segments and when combined with the ten segments of the L2 detectors a total of 300 different directions in the ecliptic plane (150 per side) are defined. Table 13 shows the geometry factor of each of the possible L1 $\bullet 2$ segment combinations evaluated for particles that stop at the endpoint of L2. For example, the geometry factor of a particle trajectory from L1A1a to L2A0 is $0.0244 \mathrm{~cm}^{2}$ sr. Note that Table 13 result does not include the energy dependence visible at the highest and lowest energies in Fig. 37. In Sect. 4.7 these detector combinations are grouped to measure particle anisotropies. Another use of Table 13 is to evaluate the effect of disabling (by command) a noisy detector should this become necessary. Finally, Table 13 includes the summed geometry factors for the $\mathrm{H}$ and $\mathrm{He}$ response when the dynamic threshold system is employed during large SEP events (see Sect. 3.6.3).

Table 13 indicates that the total geometry factor for forming an L1 $\bullet$ L2 coincidence is $4.05 \mathrm{~cm}^{2} \mathrm{sr}$. This is also the geometry factor for events telemetered to the ground. However, the onboard particle identification system ignores the four widest-angle detector combina-

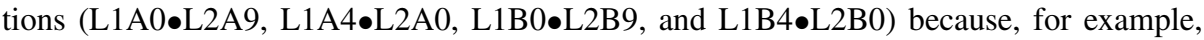
a fraction of these events may pass through L1A0, L2A0, and L3A and then exit out the side without triggering L3B. These four detector combinations are also excluded in tabulating the sectored rates (Sect. 4.7). The geometry factor for onboard particle identification is $4.0 \mathrm{~cm}^{2} \mathrm{sr}$.

\subsection{Anisotropy Measurements}

STEREO is a three-axis stabilized spacecraft that always looks at the Sun. As a result, there is no information on the arrival directions of particles except for what is provided by the sensors themselves. As discussed in Sect. 4.6, the various combinations of L1 and L2 segments define a total of 300 different directions in the ecliptic plane (150 per side). These directions have been sorted into 16 sectors, 8 of which are illustrated in Fig. 38. For sectored count rates 
Table 13 LET geometry factors

\begin{tabular}{|c|c|c|c|c|c|c|c|c|}
\hline \multicolumn{3}{|l|}{ L1AO } & \multicolumn{3}{|l|}{ L1A1 } & \multicolumn{3}{|l|}{ L1A2 } \\
\hline $\begin{array}{l}\text { L1 } \\
\text { segment }\end{array}$ & $\begin{array}{l}\text { L2 } \\
\text { segment }\end{array}$ & $\begin{array}{l}\text { Geometry } \\
\text { factor } \\
\left(\mathrm{cm}^{2} \mathrm{sr}\right)\end{array}$ & $\begin{array}{l}\text { L1 } \\
\text { segment }\end{array}$ & $\begin{array}{l}\text { L2 } \\
\text { segment }\end{array}$ & $\begin{array}{l}\text { Geometry } \\
\text { factor } \\
\left(\mathrm{cm}^{2} \mathrm{sr}\right)\end{array}$ & $\begin{array}{l}\text { L1 } \\
\text { segment }\end{array}$ & $\begin{array}{l}\text { L2 } \\
\text { segment }\end{array}$ & $\begin{array}{l}\text { Geometry } \\
\text { factor } \\
\left(\mathrm{cm}^{2} \mathrm{sr}\right)\end{array}$ \\
\hline $\mathrm{a}$ & 0 & 0.0328 & $\mathrm{a}$ & 0 & 0.0244 & $\mathrm{a}$ & 0 & 0.0157 \\
\hline $\mathrm{a}$ & 1 & 0.0267 & $\mathrm{a}$ & 1 & 0.0240 & $\mathrm{a}$ & 1 & 0.0173 \\
\hline $\mathrm{a}$ & 2 & 0.0212 & $\mathrm{a}$ & 2 & 0.0225 & $\mathrm{a}$ & 2 & 0.0186 \\
\hline $\mathrm{a}$ & 3 & 0.0168 & $\mathrm{a}$ & 3 & 0.0206 & $\mathrm{a}$ & 3 & 0.0193 \\
\hline $\mathrm{a}$ & 4 & 0.0133 & a & 4 & 0.0184 & a & 4 & 0.0196 \\
\hline $\mathrm{a}$ & 5 & 0.0106 & $\mathrm{a}$ & 5 & 0.0159 & $\mathrm{a}$ & 5 & 0.0193 \\
\hline $\mathrm{a}$ & 6 & 0.0084 & $\mathrm{a}$ & 6 & 0.0137 & $\mathrm{a}$ & 6 & 0.0183 \\
\hline $\mathrm{a}$ & 7 & 0.0068 & $\mathrm{a}$ & 7 & 0.0115 & $\mathrm{a}$ & 7 & 0.0168 \\
\hline $\mathrm{a}$ & 8 & 0.0055 & $\mathrm{a}$ & 8 & 0.0096 & $\mathrm{a}$ & 8 & 0.0150 \\
\hline $\mathrm{a}$ & 9 & 0.0044 & a & 9 & 0.0081 & a & 9 & 0.0128 \\
\hline $\mathrm{b}$ & 0 & 0.0164 & $\mathrm{~b}$ & 0 & 0.0114 & $\mathrm{~b}$ & 0 & 0.0074 \\
\hline $\mathrm{b}$ & 1 & 0.0136 & $\mathrm{~b}$ & 1 & 0.0116 & $\mathrm{~b}$ & 1 & 0.0082 \\
\hline $\mathrm{b}$ & 2 & 0.0111 & $\mathrm{~b}$ & 2 & 0.0111 & $\mathrm{~b}$ & 2 & 0.0090 \\
\hline b & 3 & 0.0090 & $\mathrm{~b}$ & 3 & 0.0103 & $\mathrm{~b}$ & 3 & 0.0095 \\
\hline b & 4 & 0.0072 & $\mathrm{~b}$ & 4 & 0.0095 & $\mathrm{~b}$ & 4 & 0.0098 \\
\hline b & 5 & 0.0058 & $\mathrm{~b}$ & 5 & 0.0083 & $\mathrm{~b}$ & 5 & 0.0098 \\
\hline b & 6 & 0.0046 & $\mathrm{~b}$ & 6 & 0.0073 & $\mathrm{~b}$ & 6 & 0.0095 \\
\hline $\mathrm{b}$ & 7 & 0.0038 & $\mathrm{~b}$ & 7 & 0.0062 & $\mathrm{~b}$ & 7 & 0.0090 \\
\hline $\mathrm{b}$ & 8 & 0.0031 & $\mathrm{~b}$ & 8 & 0.0053 & $\mathrm{~b}$ & 8 & 0.0082 \\
\hline $\mathrm{b}$ & 9 & 0.0025 & $\mathrm{~b}$ & 9 & 0.0044 & $\mathrm{~b}$ & 9 & 0.0074 \\
\hline $\mathrm{c}$ & 0 & 0.0275 & c & 0 & 0.0158 & $\mathrm{c}$ & 0 & 0.0128 \\
\hline $\mathrm{c}$ & 1 & 0.0264 & $\mathrm{c}$ & 1 & 0.0214 & $\mathrm{c}$ & 1 & 0.0150 \\
\hline $\mathrm{c}$ & 2 & 0.0224 & $\mathrm{c}$ & 2 & 0.0216 & $\mathrm{c}$ & 2 & 0.0168 \\
\hline $\mathrm{c}$ & 3 & 0.0185 & $\mathrm{c}$ & 3 & 0.0207 & $\mathrm{c}$ & 3 & 0.0183 \\
\hline $\mathrm{c}$ & 4 & 0.0151 & $\mathrm{c}$ & 4 & 0.0191 & $\mathrm{c}$ & 4 & 0.0193 \\
\hline $\mathrm{c}$ & 5 & 0.0124 & $\mathrm{c}$ & 5 & 0.0172 & $\mathrm{c}$ & 5 & 0.0196 \\
\hline $\mathrm{c}$ & 6 & 0.0100 & $\mathrm{c}$ & 6 & 0.0152 & $\mathrm{c}$ & 6 & 0.0193 \\
\hline $\mathrm{c}$ & 7 & 0.0081 & $\mathrm{c}$ & 7 & 0.0131 & $\mathrm{c}$ & 7 & 0.0186 \\
\hline $\mathrm{c}$ & 8 & 0.0067 & $\mathrm{c}$ & 8 & 0.0113 & $\mathrm{c}$ & 8 & 0.0173 \\
\hline $\mathrm{c}$ & 9 & 0.0056 & $\mathrm{c}$ & 9 & 0.0096 & $\mathrm{c}$ & 9 & 0.0157 \\
\hline \multicolumn{3}{|c|}{ a segment total $=0.1464$} & \multicolumn{3}{|c|}{ a segment total $=0.1687$} & \multicolumn{3}{|c|}{ a segment total $=0.1728$} \\
\hline \multicolumn{3}{|c|}{ b segment total $=0.0770$} & \multicolumn{3}{|c|}{ b segment total $=0.0854$} & \multicolumn{3}{|c|}{ b segment total $=0.0876$} \\
\hline \multicolumn{3}{|c|}{ c segment total $=0.1527$} & \multicolumn{3}{|c|}{ c segment total $=0.1650$} & \multicolumn{3}{|c|}{$\mathrm{c}$ segment total $=0.1728$} \\
\hline
\end{tabular}

$\mathrm{A}$ or $\mathrm{B}$ side total $=2.024$

Both side total $=4.047$
Dynamic thresholds:

1) Disable all 20 of the $\mathrm{L} 1$ outer regions

$$
0.8249 \mathrm{~cm}^{2} \mathrm{sr}
$$

2) Disable all L1 except center of L1A2b \& L1B2b

$$
0.1752 \mathrm{~cm}^{2} \mathrm{sr}
$$

3) Disable L2 segments except L2A4, L2A5; L2B4 and L2B5. Also disable the L3 segments L3Ao and L3Bo.

$0.0392 \mathrm{~cm}^{2} \mathrm{sr}$ 


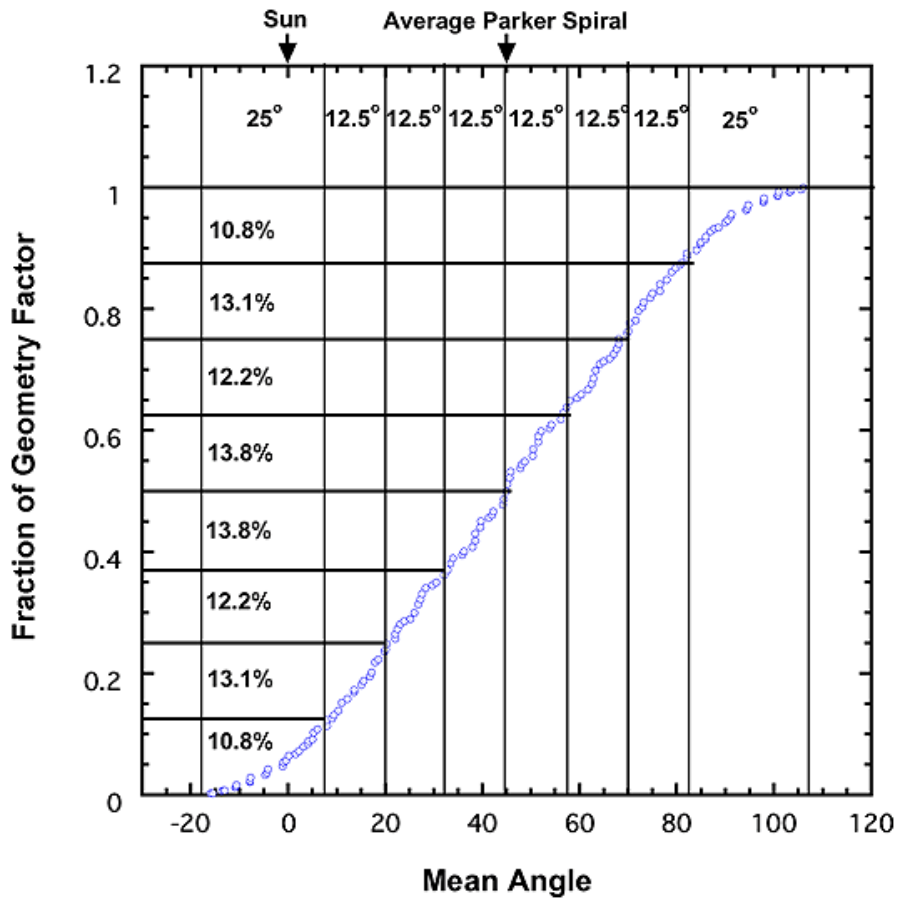

Fig. 38 The LET viewing directions are divided into eight sectors on the A-side and eight on the B-side. Shown here is the fraction of the geometry factor in each sector on the A-side. In this representation particles coming in a straight line from the Sun would arrive at $0^{\circ}$ and those arriving along the average Parker Spiral angle would arrive at $\sim 45^{\circ}$. Note that the central six sectors are $12.5^{\circ}$ wide and the width of the two outside sectors is $25^{\circ}$

Table 14 LET sectored rates

\begin{tabular}{llll}
\hline Species & Energy range & Number of sectors & Geometry factor $\left(\mathrm{cm}^{2} \mathrm{sr}\right)$ \\
\hline $\mathrm{H}$ & $4.0-6.0$ & 16 & $0.039-4.0$ \\
${ }^{3} \mathrm{He}$ & $4.0-6.0$ & 16 & $0.039-4.0$ \\
${ }^{4} \mathrm{He}$ & $4.0-6.0$ & 16 & $0.039-4.0$ \\
& $6.0-12.0$ & 16 & $0.039-4.0$ \\
$\mathrm{CNO}$ & $4.0-6.0$ & 16 & 4.0 \\
& $6.0-12.0$ & 16 & 4.0 \\
$\mathrm{NeMgSi}$ & $4.0-6.0$ & 16 & 4.0 \\
& $6.0-12.0$ & 16 & 4.0 \\
$\mathrm{Fe}$ & $4.0-6.0$ & 16 & 4.0 \\
& $6.0-12.0$ & 16 & 4.0 \\
\hline
\end{tabular}

both the front (A-side) and rear (B-side) include particles from a $129^{\circ} \times 29^{\circ}$ Field of View (FOV) with the $129^{\circ}$ fan looking along the ecliptic plane. The center of the A-side fan points at an angle that is $45^{\circ}$ west of the Sun, along the average Parker Spiral direction at 1 AU.

There are ten 16-sector rates that are read out once per minute (see Table 14). All of these count the particles identified by the onboard particle identification system described 
in Sect. 3.6.4. The CNO and NeMgSi rates sum the events along the three tracks in order to achieve improved statistical accuracy. The use of common energy intervals makes it possible to compare the pitch-angle distributions of species with the same velocity, but differing rigidities. There is complementary anisotropy data for ions and electrons provided by the SEPT sensor (Müller-Mellin et al. 2007).

During large SEP events, when the first stage of the dynamic thresholds are imposed (only L1 centers used for $\mathrm{H}$ and He; Sect. 3.6.3), the number of sectors that are triggered by $\mathrm{H}$ and $\mathrm{He}$ events will remain at 16 but the coverage in the forward and rear directions will be $127^{\circ}$ wide instead of $129^{\circ}$. During the second stage of the dynamic thresholds (when $\mathrm{H}$ and He trigger only the center segments of L1A2 and L1B2), the number of sectors that are triggered by $\mathrm{H}$ and $\mathrm{He}$ events will be reduced to six per side, and the $\mathrm{H}$ and $\mathrm{He}$ angular coverage in the ecliptic will be reduced to $\sim 62^{\circ}$ wide in the front and rear. During the third stage of the dynamic thresholds the angular coverage in the ecliptic for $\mathrm{H}$ and $\mathrm{He}$ will be only $19^{\circ}$ wide in the front and rear.

\subsection{Solar Energetic Particle Yields}

In order to estimate the yield of energetic ions that would be obtained in a very large SEP event an analysis was done of the performance of LET during the July 14, 2000 (Bastille Day), event when the intensity reached its maximum (10:00-18:00 UT on July 15). Using composite spectra compiled by Tylka et al. (2001), the count rates of $\mathrm{H}, \mathrm{He}, \mathrm{O}$, and $\mathrm{Fe}$ ions incident on the LET telescope were estimated. In addition, the singles rates of all detectors were estimated, including particles that enter through the sides of the telescope. The estimated total singles rate (summed over all detectors) is $\sim 1.6 \times 10^{5} \mathrm{~s}^{-1}$, leading to a livetime of $38 \%$ during this interval. The rate at which proton events would be analyzed [taking into account the dynamic thresholds (Sect. 3.6.3) and livetime] was estimated to be $\sim 1,200 \mathrm{~s}^{-1}$, and the analysis rate of $Z \geq 6$ heavy ions was estimated at $14 \mathrm{~s}^{-1}$. Figure 39 shows the total number of events that would be expected from Ranges 2, 3, and 4 during this 8-hr period (only those elements for which there is onboard analysis are tabulated). Note that the event numbers are adequate to construct energy spectra for all species.

There will also be pulse-height data for individual heavy ion events telemetered during this period. We expect $\sim 3$ events/s with $Z \geq 6$ to be telemetered or $\sim 20 \%$ of the total that

Fig. 39 Estimated yield of particle events that a LET would have measured during the eight hours when the counting rate from the Bastille Day SEP event was at its maximum. The effects of the dynamic thresholds and the instrument deadtime have been taken into account. Only those species identified by the onboard analysis system are shown

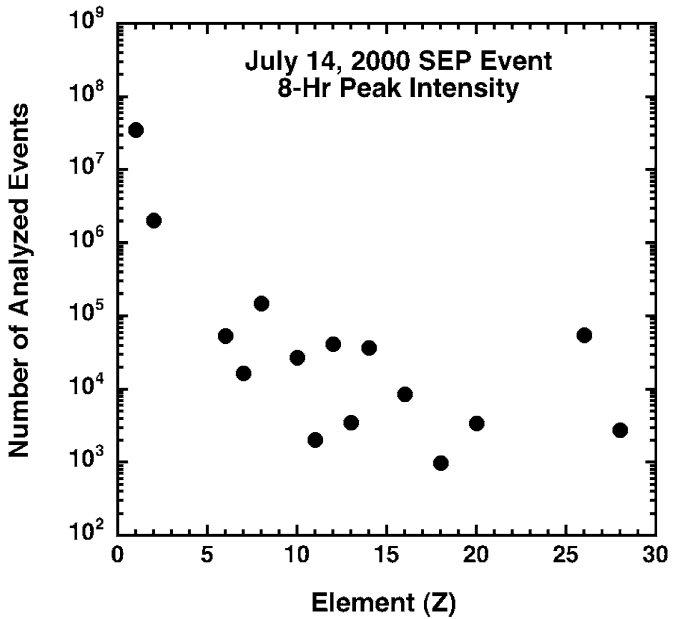


were analyzed. These data can be used to check the onboard analysis and also to analyze the abundance of species not covered by the onboard analysis. Over the course of the entire Bastille Day event we expect the number of analyzed heavy ions to be several times the totals in Fig. 39.

\subsection{In-Flight Performance}

The twin STEREO spacecraft were launched from Cape Canaveral on the evening of October 25, 2006, and placed into a lunar phasing orbit. The four instruments of the SEP suite on the Behind spacecraft were first powered up on November 13; one day later the SEP suite on the Ahead spacecraft was also turned on. As of late December both LET sensors were operating nominally. The LETs were turned on during the decay of a small energetic particle event which provided a first opportunity to test the onboard particle identification routines and obtain a cross-calibration with other $1 \mathrm{AU}$ instruments. The energy spectra for $\mathrm{H}$ and $\mathrm{He}$ measured by the LETs on November 14 were in good agreement with spectra measured by the EPAM, ULEIS, and SIS instruments on ACE, and by the EPHIN instrument on SoHO. Several small impulsive SEP events on November 21 and 22 provided further tests of the onboard particle identification system. The highly elliptical STEREO orbit during November and early December made regular transits through Earth's magnetosphere, providing an opportunity to test the "dynamic threshold" function (see Sect. 3.6.3) in an extreme radiation environment.

During early December the recovery to solar minimum conditions was interrupted by four X-class flares and associated CMEs that resulted in two large SEP events. These events populated all of the species and energy bins identified by onboard processing, providing critical in-flight calibration data for adjusting the location of the boxes in Figs. 29, 41, and 42. The observed composition and energy spectra were found to be in good agreement with simultaneous data from ACE.

\section{SEP Central}

\subsection{SEP Central Overview}

The LET, HET, and SIT sensors each require a dedicated microprocessor (MISC) for onboard data processing and sensor control. An additional MISC, called SEP Central, gathers data from all the SEP sensors, gathers SEP housekeeping data, controls the SEP SSD bias power supply, manages the SEP interface to the IMPACT IDPU, and manages the interfaces to the sensors. SEP Central also directly controls and manages data from the SEPT-NS and SEPT-E detectors, which do not incorporate dedicated microprocessors.

There are two multiplexed serial interfaces between the LET/HET/SIT sensors and SEP Central. The first interface is bidirectional, for transferring boot-code, commands, and command responses. The second interface is unidirectional, for transferring data from the instruments to SEP Central. The protocol for using these interfaces is defined in Interface Control Documents (ICDs) between each sensor and SEP Central. SEP Central controls and acquires data from SEPT-NS and SEPT-E via a separate multiplexed bidirectional serial interface.

Processed data from the microprocessors associated with LET, HET, and SIT are transmitted by those sensors to SEP Central as CCSDS packets. SEP Central timestamps these packets, recalculates the checksum, and forwards them to the IMPACT IDPU. Raw data from SEPT-NS and SEPT-E are acquired from those sensors by SEP Central. SEP Central 
compresses and packetizes these data, and forwards the packets to the IDPU. Figure 2 in Sect. 1 shows a block diagram of the SEP Sensor Suite.

The IMPACT IDPU provides the interface between the STEREO Spacecraft C\&DH system and the IMPACT instruments, including the SEP suite. All information transfer between the IMPACT instruments and the Spacecraft/Ground flows through the IDPU, including telemetry, commands, and status. SEP Central communicates with the IDPU over a dedicated serial interface. The SEP sensors and SEP Central are designed to be relatively autonomous. Once their look-up tables have been loaded and their modes and parameters set, they cycle through their data collection and transmission automatically, and provide data to the IDPU without handshaking.

\subsection{Power-On and Boot Procedures}

After power-on, SEP Central attempts to boot from page 0 of EEPROM. Subsequently, three different discrete reset commands can be sent to SEP. The first causes SEP Central to boot from page 0 of EEPROM. The second causes SEP Central to boot from page 1 of EEPROM, and the third causes SEP Central to accept boot code over the serial link to the IDPU.

The LET, HET, and SIT processors power-on whenever SEP Central powers-on. After power-on, the LET, HET, and SIT processors execute a small boot loader program stored in the processor FPGA. This boot loader manages the reception and execution of boot code received from SEP Central on the serial command link. After SEP Central boots, the process of booting LET, HET, and SIT begins via a command from the ground or the IDPU. After the sensor boot command is received, SEP Central begins the process of transferring boot code from EEPROM to the LET, HET, and SIT processors over the serial command link. All EEPROM resides within SEP Central; the sensors do not have any EEPROM.

\subsection{SEP Telemetry Data}

Telemetry data generated by the SEP sensor suite fall into five categories: science data, housekeeping data, beacon data, fill data, and command responses.

Except for beacon data, SEP telemetry data are transferred to the IMPACT IDPU as CCSDS telemetry packets. Each packet is 272 bytes in length, including an 11-byte header. An ApID code in the packet header defines the origin and content of these packets. ApID codes allocated to the SEP sensor suite are shown in Table 15 (all numbers are decimal, and all ranges are inclusive). Telemetry allocations for the SEP sensors are summarized in Table 16.

All the sensors have a data accumulation period of one minute (synchronized with each other and with the IMPACT suite). Data accumulated by the LET, HET, and SIT sensors during minute $N$ are packetized by those sensors and transferred to SEP Central during minute $N+1$. At the beginning of minute $N+2$, SEP Central combines housekeeping packets from HET, LET, and SIT with housekeeping data gathered from SEPT and from the SEP common electronics (all of the data having been collected during minute $N$ on each sensor) into a common SEP housekeeping CCSDS packet. This packet is also transmitted to the IMPACT IDPU during minute $N+2$.

Also at the beginning of $N+2$, SEP Central combines Beacon packets from HET, LET, and SIT with Beacon data gathered from SEPT (all of the data having been collected during minute $N$ on each sensor) into a SEP Beacon message block. The format of this message block is described in Sect. 8.1 of the IMPACT Serial Interface Document. It is not a CCSDS packet. SEP Central transmits this Beacon message to the IMPACT IDPU during minute 
Table 15 ApID allocations

\begin{tabular}{ll}
\hline Item & ApID \\
\hline Command response & 576 \\
SEP combined housekeeping & 577 \\
LET science & $580-587$ \\
LET housekeeping & 588 \\
LET beacon & 589 \\
HET science & $590-597$ \\
HET housekeeping & 598 \\
HET beacon & 599 \\
SIT science & $605-617$ \\
SIT housekeeping & 618 \\
SIT beacon & 619 \\
SEPT science & 600,601 \\
Fill Packet & 623 \\
Spares & $578-579,602-606,620-622$ \\
\hline
\end{tabular}

Table 16 SEP telemetry allocations

\begin{tabular}{lccccc}
\hline Data type & HET & LET & SEPT & SIT & SEP Central \\
\hline $\begin{array}{l}\text { Housekeeping } \\
\text { (bytes/minute) }\end{array}$ & 41 & 102 & 26 & 36 & 55 \\
$\begin{array}{l}\text { Beacon } \\
\text { (bytes/minute) }\end{array}$ & 28 & 46 & 44 & 24 & 2 \\
$\begin{array}{l}\text { Science } \\
\text { (packets/minute) }\end{array}$ & 6 & 16 & 2 & 12 & 0 \\
\hline
\end{tabular}

$N+2$. The IMPACT IDPU incorporates the SEP Beacon message into the IMPACT Beacon CCSDS packet during the same minute.

Finally, all science packets received by SEP Central from LET, HET, and SIT during minute $N+1$ are timestamped by SEP Central with the beginning of the data accumulation time (minute $N$ ), the packet checksums are recalculated, and the packets are forwarded to the IMPACT IDPU during minute $N+2$. Also, SEPT science packets containing data gathered by SEP Central from the SEPT sensors during minute $\mathrm{N}$ are forwarded to the IMPACT IDPU during minute $N+2$.

Acknowledgements This research was supported by the National Aeronautics and Space Administration (NASA) at the Space Radiation Laboratory (SRL) of the California Institute of Technology (under Subcontract SA2715-26309 from the University of California at Berkeley (UCB) under NASA contract NAS503131), the Jet Propulsion Laboratory (JPL), and the Goddard Space Flight Center (GSFC).

The authors thank the many individuals and organizations that contributed to the successful development of LET and SEP Central. Within SRL at Caltech we thank Janet Valenzuela, who procured and kitted electronics parts and was responsible for our documentation control; Norman Lee, who assisted the cognizant engineer in a variety of tasks; Jill Burnham, who laid out the PHASIC chip and provided engineering assistance; Minerva Calderon, who supported us in the computer and IT networks area; and Stacia Rutherford, Caprece Anderson, Pamela Nickson, Christina Davezan, Marjorie Miller, Cherylinn Rangel, and Donna Jones, who provided administrative assistance. André Jefferson of the Caltech Safety Office assisted us in moving radiation sources around the country. We wish to thank Richard Seligman, Nancy Daneau, and Gaylene Ursua of Caltech's Office of Sponsored Research for assisting us with contract issues. We thank Ray 
Yuen, Jose Lopez-Tiana, and Rudy Arvizu for procurement help. Rebekah Sims assisted in developing our Small Business Plan. Richard Paniagua and Joe Haggerty of Caltech's Physics shop assisted in making a variety of parts used in the lab and at accelerator calibrations. We thank Chris Martin and Peter Friedman of the GALEX team for the use of their excellent clean room facility.

Caltech's JPL was instrumental in many areas of the development of LET and SEP Central. Toshiro Hatake and Amin Mottiwala were responsible for the fabrication of the Caltech PHASIC hybrid; Patrick Martin, Terry Fisher, and Andy Rose supplied environmental test facilities which were superbly run by technicians Doug Perry, Geoff Laugen, Mike Sachse, Bruce Woodward, and Sandro Torres; Tom Hill provided a bake-out chamber; Dennis Kern, Wan B. Tsoi, Michael O'Connell, and Tim Werner helped with environmental test plans and reports; Charles Cruzan, Chuck Derksen, Don Schatzel, and Reza Ghaffarian assisted with failure analysis and L1 detector rework; James Arnett, David Guarino, Peyton Bates, and Guy Prichard provided management and financial accounting assistance; Pat Dillon, Glenn Anderson, Angel Garnica, Mark Hetzel, and Pat Rodriguez helped us with connector selection and harness fabrication; Gary Bivins provided expert advice for PHASIC hybrid screening; Ed Powell and Kristan Ellis helped with parts; Mark Duran helped with thermal blankets; and Brian Blakkolb assisted with contamination control. Special thanks to the engineers and technicians in the Electronics \& Packaging Section: Atul Mehta, Anarosa Arreola, Tran Ngo Lu, Martha Cortez, Yolanda Walters, Hung Truong, and Gerald Gaughen; as well as the people in Instruments \& Measurements Lab working under Lothar Kirk. Providing advice on ITAR issues were Edmond Momjian, Ann Bussone, Stephanie Lear, and Kerry Dolan.

Dean Aalami of Space Instruments was responsible for the design of the Analog Post-regulator board and the detector bias supplies. Colin Wilburn and his staff at Micron Semiconductor Ltd. fabricated the silicon detectors. Robert Kopp of Dynacs Inc. assisted with quality assurance at Caltech. C. H. Ting consulted on the design of the P24 MISC and provided the Forth operating system used in the LET and SEP Central microprocessors.

We have many to thank at UCB. Janet Luhmann is the IMPACT Principal Investigator and we thank her for her support and encouragement throughout the development period. David Curtis was the IMPACT project manager and provided expert assistance and advice to us on many levels. Peter Berg and Selda Heavner designed and fabricated the low voltage power supplies. Ron Jackson assisted us in the quality assurance area and Trish Dobson and David Weldon provided financial assistance.

The support of many individuals at GSFC was critical in the development process. Russell Harrison assisted us with the thermal vacuum testing. Bert Nahory and Traci Pluchak-Rosnack provided technician assistance. Therese Errigo was responsible for contamination control and was assisted by Kelly Henderson of Swales Aerospace. Also helping us in a variety of areas were Fred Gross, Antonio Reyes, Leslie Cusick, Jong Kadesch, Richard Katz, Mario Martins, Brian Rice, and Haydar Teymourlouei.

We are grateful to the individuals and institutions that provided facilities and assistance during our accelerator calibrations and part irradiation testing. Rocky Koga, Jeff George, and Mark Looper of Aerospace Corp. helped in both areas, providing assistance with the use of their chamber at the 88-in cyclotron at UCB and with ${ }^{60}$ Co radiation testing at Aerospace. Raman Anantaraman facilitated our accelerator calibration at the Michigan State National Superconducting Cyclotron Lab.

We are grateful to the STEREO Project Office at GSFC for their support. Nick Chrissotimos was the STEREO Project Manager and Mike Delmont was the Deputy Project Manager. We thank them for their guidance. We are especially indebted to the IMPACT Instrument Manager, Lillian Reichenthal, who became a dedicated team member, helping us in every aspect of the development and testing of LET and SEP Central. Also assisting us were Harry Culver, Larry Gibb, Jerry Hengemihle, Shane Hynes, Mike Jones, Michael Kaiser, Diane Kolos, Alexia Lyons, Tabitha Merchant, Kevin Milligan, Robert Palfrey, James Rogers, James Thurber and Steve Wasserzug.

Finally, we thank Eric Christian of NASA Headquarters who not only provided encouragement but also took shifts in monitoring our instrument during thermal vacuum testing.

\section{Appendix 1: L1 Detector Thicknesses}

As discussed in Sect. 3.4, detailed thickness maps were made for all of the flight L1 detectors in order to provide the information needed for correcting $\mathrm{dE} / \mathrm{dx}$ measurements made using these detectors for the actual thickness of the detector segment penetrated. The resulting thicknesses and their rms deviations from this mean are summarized in Table 17. For the individual detector segments these values are based on thicknesses measured at 4 points. The overall values were calculated from the entire set of 12 points, 4 on each of the 3 segments. 
Table 17 LET L1 detector thicknesses. [mean \pm rms $(\mu \mathrm{m})]$

\begin{tabular}{|c|c|c|c|c|c|}
\hline $\begin{array}{l}\text { Detector } \\
\text { position }\end{array}$ & $\begin{array}{l}\text { Serial } \\
\text { number }\end{array}$ & $\begin{array}{l}\text { Overall } \\
\text { thickness }\end{array}$ & a segment & b segment & c segment \\
\hline \multicolumn{6}{|c|}{ Ahead spacecraft } \\
\hline L1A0 & L1-51 & $22.22 \pm 0.76$ & $22.33 \pm 0.41$ & $22.83 \pm 0.48$ & $21.50 \pm 0.72$ \\
\hline L1A1 & $2250-2-1$ & $28.49 \pm 0.55$ & $28.39 \pm 0.70$ & $28.65 \pm 0.11$ & $28.42 \pm 0.75$ \\
\hline L1A2 & $2250-2-2$ & $29.92 \pm 0.37$ & $29.59 \pm 0.31$ & $30.00 \pm 0.21$ & $30.18 \pm 0.32$ \\
\hline L1A3 & $\mathrm{L} 1-12$ & $22.52 \pm 0.47$ & $22.71 \pm 0.55$ & $22.18 \pm 0.17$ & $22.67 \pm 0.48$ \\
\hline L1A4 & L1-59 & $24.52 \pm 0.55$ & $24.22 \pm 0.60$ & $24.57 \pm 0.61$ & $24.79 \pm 0.38$ \\
\hline L1B0 & L1-22 & $22.08 \pm 0.57$ & $21.72 \pm 0.23$ & $21.84 \pm 0.35$ & $22.70 \pm 0.50$ \\
\hline L1B1 & L1-28 & $27.33 \pm 0.38$ & $27.15 \pm 0.46$ & $27.45 \pm 0.39$ & $27.39 \pm 0.31$ \\
\hline L1B2 & $\mathrm{L} 1-58$ & $23.23 \pm 0.33$ & $23.43 \pm 0.27$ & $22.99 \pm 0.27$ & $23.28 \pm 0.33$ \\
\hline L1B3 & L1-49 & $23.80 \pm 0.68$ & $23.64 \pm 0.96$ & $23.92 \pm 0.63$ & $23.84 \pm 0.58$ \\
\hline L1B4 & L1-06 & $22.41 \pm 0.38$ & $22.73 \pm 0.32$ & $22.35 \pm 0.31$ & $22.14 \pm 0.32$ \\
\hline \multicolumn{6}{|c|}{ Behind spacecraft } \\
\hline L1A0 & L1-36 & $22.36 \pm 0.76$ & $22.71 \pm 0.97$ & $21.73 \pm 0.22$ & $22.66 \pm 0.55$ \\
\hline L1A1 & L1-02 & $26.21 \pm 0.47$ & $26.22 \pm 0.59$ & $26.31 \pm 0.47$ & $26.12 \pm 0.44$ \\
\hline L1A2 & $2250-2-3$ & $29.59 \pm 0.46$ & $29.85 \pm 0.20$ & $29.82 \pm 0.17$ & $29.12 \pm 0.51$ \\
\hline L1A3 & L1-08 & $24.46 \pm 0.43$ & $24.47 \pm 0.46$ & $24.19 \pm 0.30$ & $24.72 \pm 0.41$ \\
\hline L1A4 & L1-24 & $25.62 \pm 0.63$ & $25.68 \pm 0.42$ & $25.98 \pm 0.65$ & $25.20 \pm 0.65$ \\
\hline L1B0 & L1-05 & $22.40 \pm 0.57$ & $21.92 \pm 0.54$ & $22.53 \pm 0.27$ & $22.74 \pm 0.58$ \\
\hline L1B1 & L1-35 & $27.14 \pm 0.38$ & $27.16 \pm 0.50$ & $27.20 \pm 0.39$ & $27.06 \pm 0.34$ \\
\hline L1B2 & L1-37 & $23.13 \pm 0.53$ & $23.74 \pm 0.63$ & $22.82 \pm 0.17$ & $22.86 \pm 0.15$ \\
\hline L1B3 & L1-19 & $23.56 \pm 0.52$ & $23.74 \pm 0.63$ & $23.43 \pm 0.40$ & $23.52 \pm 0.61$ \\
\hline L1B4 & $2250-3-1$ & $22.39 \pm 0.36$ & $22.60 \pm 0.28$ & $22.45 \pm 0.19$ & $22.11 \pm 0.43$ \\
\hline
\end{tabular}

\section{Appendix 2: Event Classes and Coincidence Conditions}

There are ten different classes of events defined in LET, as summarized in Table 18. These classes are defined by the event tag bits L1A (logical OR of the 15 L1A segments), L2A (logical OR of the ten L2A segments), and L3A (logical OR of the 15 L3A segments), with similar definitions for the L1B, L2B, and L3B tags. In order to resolve whether a PENA? event should be classified as PENA or ERROR, the onboard software examines the L2B hits and decides if they are due to cross-talk from L3B. If they are, then the event is PENA. If not, the event is rejected for onboard processing and sent to priority buffer 26 .

Table 19 gives the coincidence conditions that lead to the ten different classes of events. Events are classified based on the pattern of hits in each layer of the LET instrument. A " 1 " in any cell in the table signifies one or more hits in that layer. The number of events assigned to each class is counted and the totals are telemetered each minute. (These are the coincidence rates.) Note: the ordering of the layers in this table is for human readability. It does not reflect the ordering of the tag bits in the raw event data. 
Table 18 LET event classes

\begin{tabular}{ll}
\hline Event Class & Definition \\
\hline L12A & Range 2 A-side events \\
L123A & Range 3 A-side events \\
PENA & Range 4 A-side events \\
L12B & Range 2 B-side events \\
L123B & Range 3 B-side events \\
PENB & Range 4 B-side events \\
2TEL & Coincidence on both sides; rejected for onboard processing \\
PENA? & Needs subsequent processing to decide if PENA or ERROR event \\
PENB? & Needs subsequent processing to decide if PENB or ERROR event \\
ERROR & Rejected for onboard processing; sent to priority buffer 26 \\
\hline
\end{tabular}

Table 19 Onboard classification of PHA events

\begin{tabular}{|c|c|c|c|c|c|c|}
\hline L1A & L2A & L3A & L3B & L2B & L1B & Class \\
\hline 1 & 1 & 0 & 0 & 0 & 0 & L12A \\
\hline 1 & 1 & 1 & 0 & 0 & 0 & L123A \\
\hline 1 & 1 & 0 & 0 & 0 & 1 & L12A \\
\hline 1 & 1 & 1 & 0 & 0 & 1 & L123A \\
\hline 1 & 1 & 0 & 0 & 1 & 0 & L12A \\
\hline 1 & 1 & 1 & 0 & 1 & 0 & L123A \\
\hline 0 & 0 & 0 & 0 & 1 & 1 & L12B \\
\hline 1 & 0 & 0 & 0 & 1 & 1 & L12B \\
\hline 0 & 1 & 0 & 0 & 1 & 1 & L12B \\
\hline 1 & 1 & 0 & 0 & 1 & 1 & 2TEL \\
\hline 0 & 0 & 1 & 0 & 1 & 1 & L12B \\
\hline 1 & 0 & 1 & 0 & 1 & 1 & L12B \\
\hline 0 & 1 & 1 & 0 & 1 & 1 & L12B \\
\hline 1 & 1 & 1 & 0 & 1 & 1 & 2TEL \\
\hline 1 & 1 & 0 & 1 & 0 & 0 & L12A \\
\hline 1 & 1 & 1 & 1 & 0 & 0 & PENA \\
\hline 1 & 1 & 0 & 1 & 0 & 1 & L12A \\
\hline 1 & 1 & 1 & 1 & 0 & 1 & PENA \\
\hline 1 & 1 & 0 & 1 & 1 & 0 & L12A \\
\hline 1 & 1 & 1 & 1 & 1 & 0 & PENA? \\
\hline 0 & 0 & 0 & 1 & 1 & 1 & L123B \\
\hline 1 & 0 & 0 & 1 & 1 & 1 & L123B \\
\hline 0 & 1 & 0 & 1 & 1 & 1 & L123B \\
\hline 1 & 1 & 0 & 1 & 1 & 1 & 2TEL \\
\hline 0 & 0 & 1 & 1 & 1 & 1 & PENB \\
\hline 1 & 0 & 1 & 1 & 1 & 1 & PENB \\
\hline 0 & 1 & 1 & 1 & 1 & 1 & PENB? \\
\hline 1 & 1 & 1 & 1 & 1 & 1 & 2TEL \\
\hline \multicolumn{6}{|c|}{ All other combinations } & ERROR \\
\hline
\end{tabular}




\section{Appendix 3: Rates, Particle IDs, and Event Data}

The LET Science Frame is envisioned as a stream of bytes. Each minute, this stream is formatted into 16 CCSDS packets and telemetered via SEP Central. When data elements span more than one byte, the elements are transmitted in order of high byte to low byte. Both rates and PHA event data are present in the LET Science Frame (see Fig. 40). LET rates data include livetimes, detector singles and coincidence rates, onboard event processing rates, priority buffer counters, science rates, background rates, and look direction rates. The science rates are counts per minute of elements in various energy bins, divided between penetration ranges in the LET instrument. All rates are compressed to 16 bits with a modified biased exponent, hidden one algorithm with a 12-bit mantissa and a 4-bit exponent. Table 20 summarizes the rates and particle PHA event data that are telemetered each minute by LET. The format of the PHA event data is described later in this appendix.

As described in Sect. 3.6.4, valid particle events (e.g., those having straight-trajectory single detector hits in a given detector layer of the instrument) are sorted into penetration ranges ( 2 through 4 ) in the instrument. After performing gain, angle, and thickness corrections, the algorithm determines species by mapping delta- $E$ vs. $E^{\prime}$ signals onto response matrices. The matrix for Range 3 is given in Fig. 29 in Sect. 3.6.4, and the matrices for Range 2 and Range 4 are shown here in Figs. 41 and 42. Each matrix is a $128 \times 400$ array logarithmically spanning $\Delta E$ vs. $E^{\prime}$ space. Each array element is an integer identifying an element or helium isotope (1-16) or background or a STIM box (127-139).

Each colored region on the matrix figures represents a particle region (e.g., $\mathrm{H},{ }^{3} \mathrm{He},{ }^{4} \mathrm{He}$, $\mathrm{C}, \mathrm{N}, \mathrm{O}$, etc., depending on range), a background region, or a STIM box. The matrices assign a number to each particle species, according to Table 21 . Thus, a particle is mapped via $\Delta E$

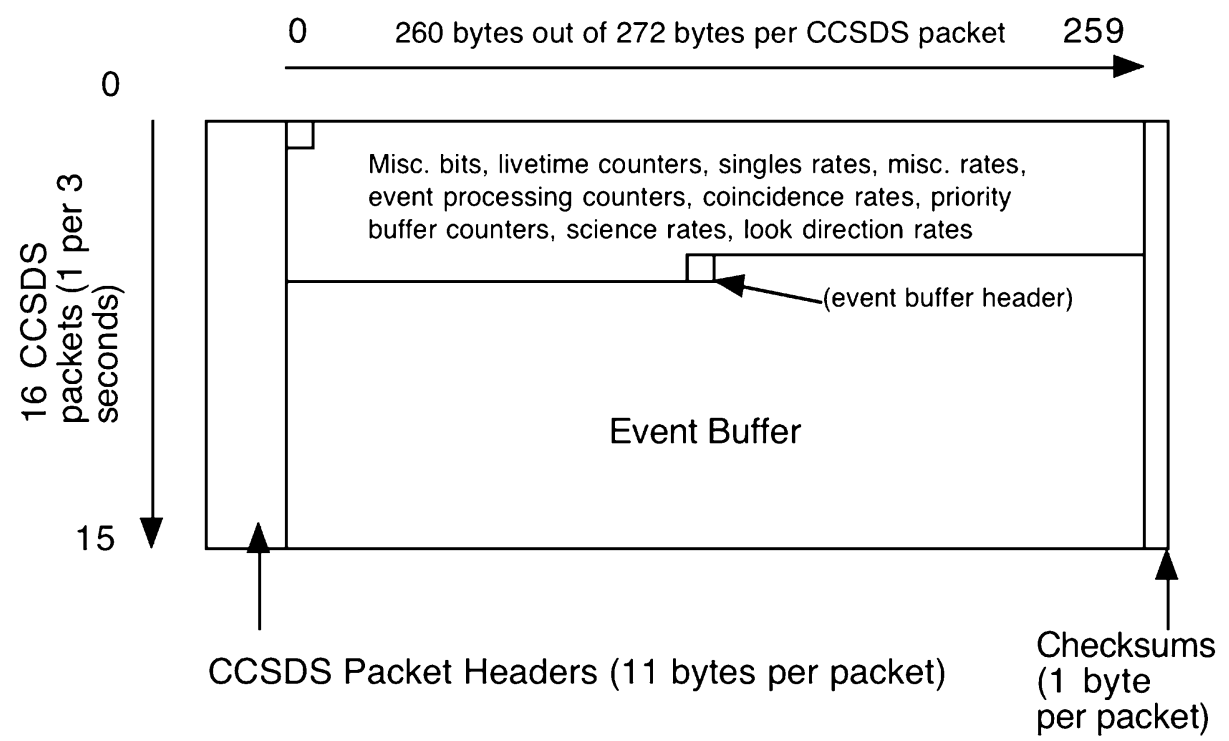

Fig. 40 LET Science Frame Overview, with CCSDS packet headers and checksums. LET Science Frame Header is unlabeled at upper left. (Data elements not to horizontal scale.) LET transmits 16 Science Frame packets to SEP Central, followed by four additional, nulled packets that SEP Central does not forward to telemetry. If more bandwidth is allocated to LET, these additional packets could be added to the LET Science Frame format 
Table 20 LET science format summary

\begin{tabular}{ll}
\hline Byte \# & Description \\
\hline $0-4$ & Header \\
$5-6$ & Miscellaneous bits (MISCBITS) \\
$7-24$ & Livetime counters (ERATES) \\
$25-240$ & Singles rates (SNGRATES) \\
$241-250$ & Miscellaneous rates (MISCRATES) \\
$251-282$ & Event processing counters (EVPRATES) \\
$283-306$ & Coincidence rates \\
$307-364$ & Priority buffer rates (BUFRATES) \\
$365-628$ & Range 2 science rates (L2FGRATES) \\
$629-652$ & Range 2 background and STIM rates (L2BGRATES) \\
$653-986$ & Range 3 science rates (L3FGRATES) \\
$987-1,010$ & Range 3 background and STIM rates (L3BGRATES) \\
$1,011-1,076$ & Range 4 science rates (PENFGRATES) \\
$1,077-1,106$ & Range 4 background and STIM rates (PENBGRATES) \\
$1,107-1,426$ & Look direction (sectored) rates (SECTRATES) \\
$1,427-1,428$ & Event buffer header \\
$1,429-4,159$ & Event records buffer \\
\hline &
\end{tabular}

Fig. 41 The Range 2 (L1 vs. L2, or L1L2) LET matrix.

Foreground elements are $\mathrm{H},{ }^{3} \mathrm{He}$, ${ }^{4} \mathrm{He}, \mathrm{C}, \mathrm{N}, \mathrm{O}, \mathrm{Ne}, \mathrm{Mg}, \mathrm{Si}, \mathrm{S}, \mathrm{Ar}$, $\mathrm{Ca}$, and $\mathrm{Fe}$, and these are shown in alternating green and yellow bands. Background regions are blue, and live STIM boxes are shown in orange. The data points are from the December 13, 2006, SEP event. Note that only 1 in 100 of the events below $\mathrm{C}$ have been plotted

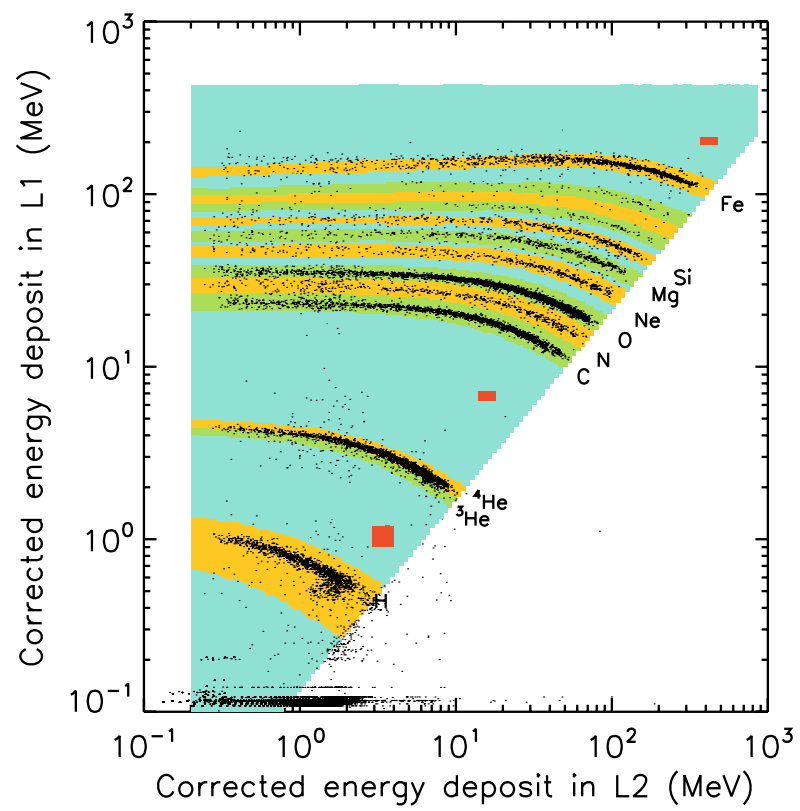

vs. $E^{\prime}$ onto a matrix, and from its location on the matrix file, it is assigned a matrix value $(1-16,128-141)$. If a particle is identified as a selected element for its penetration range (matrix value 1-16), its energy is calculated from the sum of deposited energies in each detector layer, and its energy/nucleon is calculated from the total energy and assumed mass. 
Fig. 42 The Range 4 (L3A vs. L3B, or L3A vs. L3B) LET matrix. Foreground elements are $\mathrm{H},{ }^{4} \mathrm{He}, \mathrm{C}, \mathrm{N}, \mathrm{O}, \mathrm{Ne}, \mathrm{Mg}, \mathrm{Si}$, and $\mathrm{Fe}$, and these are shown in alternating green and yellow bands. Background and RNG4 penetrating particle regions are blue, and live STIM boxes are shown in orange. The data points are from the December 13, 2006, SEP event. Note that only 1 in 100 of the events below $\mathrm{C}$ have been plotted

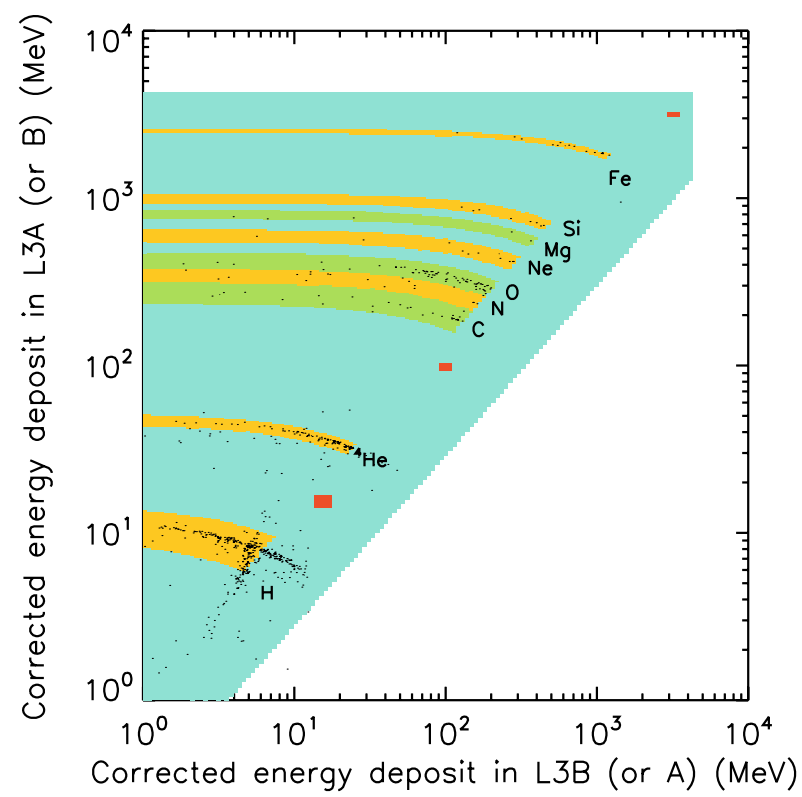

Table 21 Matrix value and particle ID assignments

\begin{tabular}{lllll}
\hline Element & Matrix value & $\begin{array}{l}\text { Range 2 } \\
\text { particle IDs }\end{array}$ & $\begin{array}{l}\text { Range 3 } \\
\text { particle IDs }\end{array}$ & $\begin{array}{l}\text { Range 4 } \\
\text { particle IDs }\end{array}$ \\
\hline $\mathrm{H}$ & 1 & $0-8$ & $0-10$ & $0-2$ \\
${ }^{3} \mathrm{He}$ & 2 & $9-17$ & $11-19$ & \\
${ }^{4} \mathrm{He}$ & 3 & $18-26$ & $20-30$ & $3-5$ \\
$\mathrm{C}$ & 4 & $27-35$ & $31-41$ & $6-9$ \\
$\mathrm{~N}$ & 5 & $36-44$ & $42-51$ & $10-13$ \\
$\mathrm{O}$ & 6 & $45-53$ & $52-61$ & $14-16$ \\
$\mathrm{Ne}$ & 7 & $54-63$ & $62-71$ & $17-20$ \\
$\mathrm{Na}$ & 8 & & $72-81$ & \\
$\mathrm{Mg}$ & 9 & $64-74$ & $82-92$ & $21-24$ \\
$\mathrm{Al}$ & 10 & & $93-103$ & \\
$\mathrm{Si}$ & 11 & $75-85$ & $104-114$ & $25-28$ \\
$\mathrm{~S}$ & 12 & $86-95$ & $115-125$ & \\
$\mathrm{Ar}$ & 13 & $96-106$ & $126-136$ & \\
$\mathrm{Ca}$ & 14 & $107-117$ & $137-146$ & \\
$\mathrm{Fe}$ & 15 & $118-130$ & $147-156$ & $29-31$ \\
$\mathrm{Ni}$ & 16 & & $157-166$ & \\
Other (e.g. STIM, & $128-141$ & 255 & 255 & 255 \\
background) & & & & \\
\hline
\end{tabular}


Table 22 Event record header (ERH)

\begin{tabular}{|c|c|c|}
\hline Data & Bit & Note \\
\hline Particle ID & $0-7$ & $\begin{array}{l}=\text { particle ID if the particles are sorted by the LET Matrices as } \\
\text { "foreground" particles. (= } 255 \text { if the particles are not sorted by the } \\
\text { matrices or are identified as background particles). Allows matrices } \\
\text { to ID particles by range (matrix), species, and energy. See } \\
\text { Appendix 2, Table } 18 \text { for lists of Particle IDs. }\end{array}$ \\
\hline $\begin{array}{l}\text { Priority buffer } \\
\text { number }\end{array}$ & $8-12$ & $\begin{array}{l}\text { See Sect. 3.6.5, Table } 9 \text { for a list of Priority Buffer Numbers and their } \\
\text { descriptions. }\end{array}$ \\
\hline L1A tag & 13 & Indicates an L1A detector contributed to the coincidence trigger. \\
\hline L2A tag & 14 & Indicates an L2A detector contributed to the trigger. \\
\hline L3A tag & 15 & Indicates an $\mathrm{L} 3 \mathrm{~A}$ detector contributed to the trigger. \\
\hline L1B tag & 16 & Indicates an L1B detector contributed to the trigger. \\
\hline L2B tag & 17 & Indicates an L2B detector contributed to the trigger. \\
\hline L3B tag & 18 & Indicates an L3B detector contributed to the trigger. \\
\hline STIM tag & 19 & Flags a STIM event. \\
\hline HAZ tag & 20 & Hazard flag. \\
\hline $\begin{array}{l}\text { Time tag } \\
\text { (latency) }\end{array}$ & $21-24$ & $\begin{array}{l}\text { Duplicates the } 4 \text { least significant bits of an onboard minutes counter; } \\
\text { used to identify event latency. }\end{array}$ \\
\hline $\mathrm{A} / \mathrm{B}$ event tag & 25 & $\mathrm{~A}=0, \mathrm{~B}=1$ \\
\hline \# Unread ADCs & $26-28$ & \# hit ADCs not included in the Event Record. Saturates at 7. \\
\hline $\begin{array}{l}\text { Extended header } \\
\quad \text { flag }\end{array}$ & 29 & $\begin{array}{l}=1 \text { if an additional header byte (or set of bytes) is appended to this } \\
\text { header. }\end{array}$ \\
\hline STIM block flag & 30 & $=1$ if STIM Information Block is included in this event. \\
\hline Culling flag & 31 & $=1$ if number of ADCs culled from this event is nonzero. \\
\hline
\end{tabular}

The final particle ID is then determined from species, energy, and range, with separate tables of particle ID values used for each penetration range. Energy bin boundaries are shown in Fig. 30. Particle ID values range from 0 to 166, depending on range. STIM events, events falling into the background regions of the matrices, and invalid events are assigned particle IDs of 255 .

LET also transmits pulse height data for a sample of all events processed through the particle identification and priority system; see Sects. 3.6.4 and 3.6.5. These events are packaged in variable-length event records, including an event record header, an optional extended header, an optional STIM information block, and a number of event record ADC fields. The 32-bit event record header summarized in Table 22 contains various tags as well as priority buffer number and particle ID number. The event record header is also shown graphically in Fig. 43a, reflecting the high-byte-first ordering.

The optional 16-bit extended header block contains the location of the event on the event matrices. If the event was not processed through a matrix, its location is 0,0 . If the extended header block is included in an event record, it is appended after the ADC fields.

The optional 16-bit STIM information block contains a time counter indicating the time during the accumulation minute at which the STIM event was generated. The block also includes bits indicating the DAC settings for the STIM event, i.e., the DAC levels and which ADCs were stimulated. If included, this block would be appended after the ADC fields or after the optional extended header block.

The rest of an event record is filled with 20-bit ADC fields, one field for each ADC included in the event. The ADC field contents are summarized in Table 23 and Fig. 43b. Gen- 


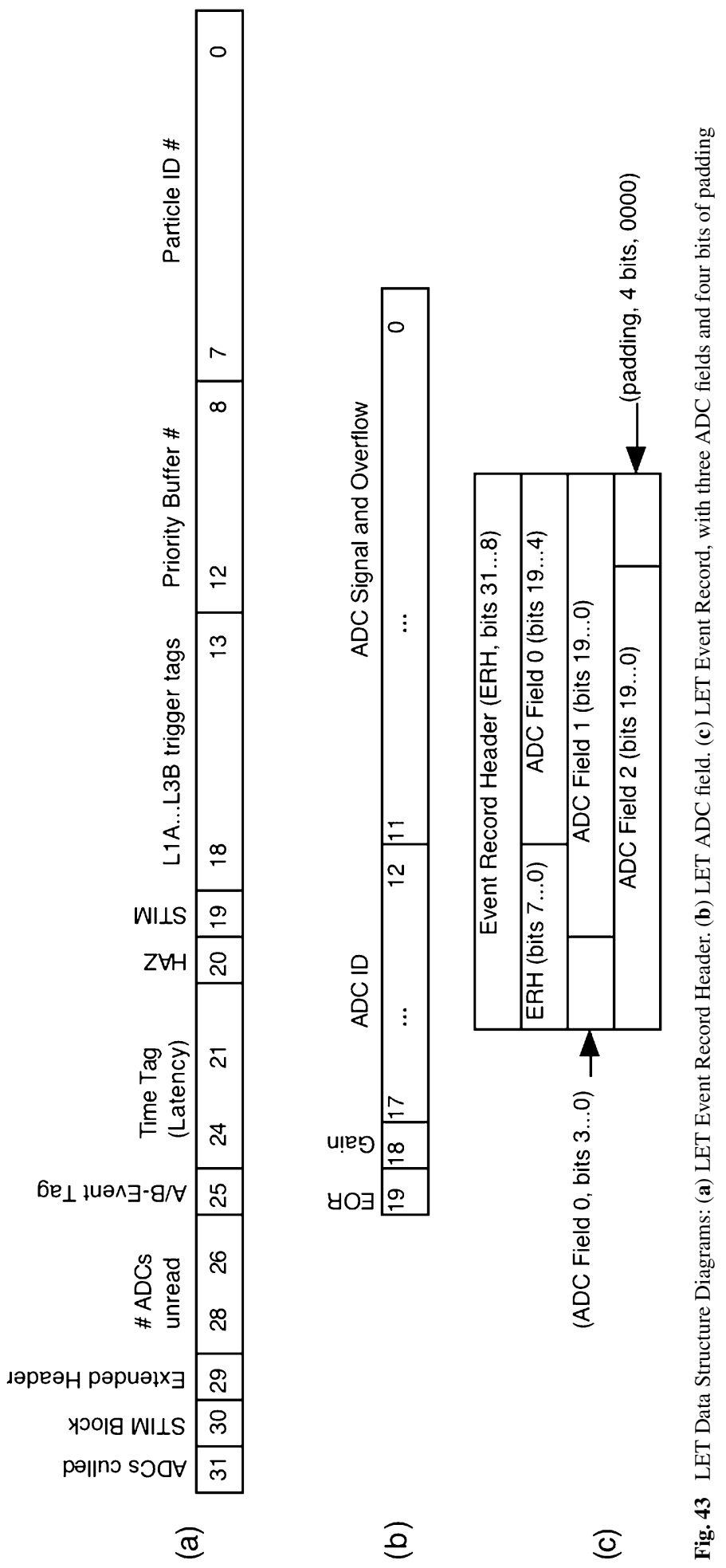


Table 23 ADC field

\begin{tabular}{lll}
\hline Data & Bits & Note \\
\hline ADC signal & $0-11$ & 11 bits signal, 1 bit overflow \\
ADC/Detector ID & $12-17$ & 54 detectors maximum \\
Low/High gain & 18 & $0=$ low gain, $1=$ high gain \\
Last hit flag (End of Record, EOR) & 19 & Set to 1 for the last ADC in an event, 0 for \\
& & all other ADCs \\
\hline
\end{tabular}

erally, the event record contains only those ADC fields corresponding to the pulse heights used by the onboard software to identify the particle. More ADCs may be included for unsorted events, and if large numbers of detectors reported pulse heights, some are dropped and counted only in the event record header. Finally, the LET sensor has a VERBOSE mode in which both low- and high-gain signals from a given detector may be transmitted.

Finally, an event record is padded with additional zero bits as needed to align each event record along byte boundaries in the Science Frame. A sample event record with three ADC fields and without a STIM information block or extended header is shown diagrammatically in Fig. 43c. If a STIM information block and/or an extended header were included, any necessary padding would be appended after these blocks.

\section{Appendix 4: LET Variables}

Table 24 defines LET software variables and tables used to control features of the real-time particle identification system described in Sect. 3.6.4. All variables in Table 24 with units of ADC channels are compared with ADC pulse-height data after the onboard software has performed DC offset-subtraction. The values of all variables in the tables may be changed by command.

The following tables used by the real-time particle ID system may also be changed by command.

The index into the following seven tables is the six bits of chip/PHA address contained in each PHA word read in from the PHASIC (two bits chip and four bits pha address). The contents of these tables are different for each Flight Unit.

FSETLOW: LET Low-gain DC offset corrections, in ADC channels.

FSETHIGH: LET high-gain DC offset corrections, in ADC channels.

GAINLOW: LET Low-gain gain correction lookup table. Each entry is (int)(256* CF), where $\mathrm{CF}$ is a multiplicative correction factor that converts an offset-corrected PHA signal to a nominal gain-level. For each entry, $\mathrm{CF}$ is scaled so that gain-corrected low-gain signals from L1, L2, and L3 all have the same energy-scale.

GAINHIGH: LET Low-gain gain correction lookup table. Each entry is (int) $(256 * \mathrm{CF})$, where $\mathrm{CF}$ is a multiplicative correction factor that converts an offset-corrected PHA signal to a nominal gain-level. For each entry, $\mathrm{CF}$ is scaled so that gain-corrected high-gain signals from L1, L2, and L3 all have the same energy scale. Note: the nominal factor of 20 difference between high-gain and low-gain signals is handled separately from these tables.

THICKNES: Each entry is (int) $(256 * \mathrm{CF})$, where $\mathrm{CF}$ is a multiplicative correction factor that converts a PHA signal to the signal that would be expected for a detector of nominal thickness. $\mathrm{CF}=\left[L / L_{0}\right]^{1 / \alpha}$, where $\alpha$ differs for L1, L2, L3 (see the following cosine tables).

WINCORR2, WINCORR3: LET window correction lookup table. Each entry is (int) $(256 * \mathrm{CF})$. Only L1 signals need to be corrected to account for the thickness of the 
Table 24 LET onboard event processing tunable variables

\begin{tabular}{|c|c|c|c|}
\hline Variable name & $\begin{array}{l}\text { Default } \\
\text { value }\end{array}$ & Units & Description \\
\hline REJHAZ & TRUE & Boolean & $\begin{array}{l}\text { if TRUE, hazard-tagged events are rejected for onboard } \\
\text { processing }\end{array}$ \\
\hline FCULL & TRUE & Boolean & $\begin{array}{l}\text { If TRUE, cross-talk hits are culled from telemetered } \\
\text { events }\end{array}$ \\
\hline FNMAX & 20 & ADCs/event & $\begin{array}{l}\text { Max number of ADCs that will be formatted per event, } \\
\text { (independent of FCULL) }\end{array}$ \\
\hline CHKR3E? & TRUE & Boolean & $\begin{array}{l}\text { If TRUE, enables checking of L1 and L } 2 \text { energies for } \\
\text { Range } 3 \text { events }\end{array}$ \\
\hline CHKL1L2R? & TRUE & Boolean & $\begin{array}{l}\text { If TRUE, enables checking of L1/L2 energy ratio for } \\
\text { Range } 3 \text { and } 4 \text { events }\end{array}$ \\
\hline L2SHIFT & 4 & - & $\begin{array}{l}\text { sets minimum allowed value of } \mathrm{L} 1 / \mathrm{L} 2 \text { energy ratio: } 4 \\
\text { implies min_ratio }=1 / 16,3 \text { implies min_r }=1 / 8,2 \\
\text { implies min_r }=1 / 4 \text {, etc. }\end{array}$ \\
\hline STFSET2 & 8 & Matrix cells & $\begin{array}{l}\text { \# of cells to shift EPrime IDX if a real event falls in } \\
\text { Range } 2 \text { STIM box }\end{array}$ \\
\hline STFSET3 & 8 & Matrix cells & $\begin{array}{l}\text { \# of cells to shift EPrime IDX if a real event falls in } \\
\text { Range } 3 \text { STIM box }\end{array}$ \\
\hline STFSET4 & 22 & Matrix cells & $\begin{array}{l}\text { \# of cells to shift EPrime IDX if a real event falls in } \\
\text { Range } 4 \text { STIM box }\end{array}$ \\
\hline CHKTAGS? & TRUE & Boolean & $\begin{array}{l}\text { If TRUE, enables onboard checking of tags generated by } \\
\text { front-end logic }\end{array}$ \\
\hline FIXTAGS? & TRUE & Boolean & $\begin{array}{l}\text { If TRUE, enables onboard fixing of tags generated by } \\
\text { front-end logic }\end{array}$ \\
\hline REJL3TH & & ADC channels & $\begin{array}{l}\text { used to test for the presence of an L3 signal in events } \\
\text { rejected for onboard processing. If an L } 3 \text { signal above } \\
\text { REJL3TH is present, the event is assigned to priority } \\
\text { buffers } 9 \text { or } 20 \text {. If not, the event is assigned to priority } \\
\text { buffers } 10 \text { or } 21 .\end{array}$ \\
\hline L2DELOWZLIM & & ADC channels & $\begin{array}{l}\text { used to test if a Range } 2 \text { event is lo- } Z \text { or Hi- } Z \text {. This is the } \\
\text { cut on the L1 (delta_E) signal }\end{array}$ \\
\hline L2EPLOWZLIM & & ADC channels & $\begin{array}{l}\text { used to test if a Range } 2 \text { event is lo- } Z \text { or Hi- } Z \text {. This is the } \\
\text { cut on the L2 (Eprime) signal }\end{array}$ \\
\hline L3DELOWZLIM & & ADC channels & $\begin{array}{l}\text { used to test if a Range } 3 \text { event is lo- } Z \text { or Hi- } Z \text {. This is the } \\
\text { cut on the L2 (delta_E) signal }\end{array}$ \\
\hline L3EPLOZLIM & & ADC channels & $\begin{array}{l}\text { used to test if a Range } 3 \text { event is lo- } Z \text { or Hi- } Z \text {. This is the } \\
\text { cut on the L3 (Eprime) signal }\end{array}$ \\
\hline PDELOWZLIM & & ADC channels & $\begin{array}{l}\text { used to test if a Range } 4 \text { event is lo- } Z \text { or Hi- } Z \text {. This is the } \\
\text { cut on the L3 }(\Delta E) \text { signal }\end{array}$ \\
\hline
\end{tabular}


Table 24 (Continued)

\begin{tabular}{llll}
\hline Variable name & $\begin{array}{l}\text { Default } \\
\text { value }\end{array}$ & Units & Description \\
\hline PEPLOWZLIM & ADC channels & $\begin{array}{l}\text { used to test if a Range } 4 \text { event is lo- } Z \text { or Hi- } Z \text {. This is the } \\
\text { cut on the L3 (Eprime) signal }\end{array}$ \\
XTALKTH12 & ADC channels & $\begin{array}{l}\text { L1, L2 cross-talk threshold }- \text { L1or L2 pulse-heights } \\
\text { above this are not cross-talk }\end{array}$ \\
XTALKTH3 & ADC channels & $\begin{array}{l}\text { L3 cross-talk threshold }- \text { L3 pulse-heights above this are } \\
\text { not cross-talk }\end{array}$ \\
CRXTALKTH & ADC channels & $\begin{array}{l}\text { threshold above which an ADC is likely to produce } \\
\text { cross-talk }\end{array}$ \\
\hline
\end{tabular}

LET windows, so the L2 and L3 entries are unused. WINCORR2 is used for Range 2 events. WINCORR3 is used for Range 3 and 4 events.

The index into the following nine tables is (L1ID «2) | L2ID. So, each entry refers to a particular L1-L2 detector combination.

R2COSINES, R3COSINES, PENCOSINES: Angle correction lookup tables for Range 2, Range 3, and Range 4 (PEN) events. Each entry is (int) $\left(256 *(\cos (q))^{1 / \alpha}\right)$. For Range 2, $\cos \theta$ is the cosine of the angle between the L1-perpendicular and the line between the centers of the L1 and L2 detectors. For Range 3 and 4, $\cos \theta$ is the $\operatorname{cosine}$ of the angle between the L2-perpendicular and the line between the centers of the L1 and L2 detectors. $\alpha$ is different for each range: currently 1.36, 1.65, 1.77 for Ranges 2, 3, and 4 respectively.

EXCLUDER2A, EXCLUDER2B: For Range 2 events, if a table entry is set to 1, then that L1-L2 combination will be excluded from onboard processing for A-side (B-side) events.

EXCLUDER3I: For Range 3 events with L3-inner hit, table entries are set to 1 if the L1L2 combination is inconsistent with an L3-inner hit, or if the event might be a penetrating particle escaping out the side without hitting the R4 (PEN) detector.

EXCLUDER3O: For Range 3 events with L3-outer hit, table entries are set to 1 if the L1L2 combination is inconsistent with an L3-outer hit, or if the event might be a penetrating particle escaping out the side without hitting the R4 (PEN) detector.

SECTORID: Identifies the sector that any given L1-L2 combination belongs to.

TAGTABLE : This table is indexed by the six tag bits of an ADC word. Each entry is a constant that defines the type of event that the tag bits signify: L12A, L123A, L12B, L123B, PENA, PENB, PENA?, PENB?, 2TEL, ERR.

\section{References}

W.E. Althouse et al., IEEE Trans. Geosci. Electron. GE-16, 204 (1978)

J.-L. Bougeret et al., Space Sci. Rev. (2007, this issue)

L.F. Burlaga et al., Science 309, 2027 (2005)

CCSDS, in Packet Telemetry Recommendation for Space Data System Standards (CCSDS, Washington, 2000). 102.0-B-5, Blue Book, Issue5

H.V. Cane, D. Lario, Space Sci. Rev. (2006). doi:10.1007/s11214-006-9011-3

H.V. Cane, W.C. Erickson, N.P. Prestage, J. Geophys. Res. (2002). doi:10.1029/2001JA000320

H.V. Cane et al., Geophys. Res. Lett. (2003). doi:10.1029/2002GL016580

H.V. Cane et al., J. Geophys. Res. (2006). doi:10.1029/2005JA011071

C.M.S. Cohen et al., Geophys. Res. Lett. 26, 2697 (1999) 
C.M.S. Cohen et al., J. Geophys. Res. (2005). doi:10.1029/2005JA011004

C.M.S. Cohen et al., Space Sci. Rev. (2007). doi:10.1007/s11214-007-9218-y

W.R. Cook et al., Custom analog VLSI for the Advanced Composition Explorer (ACE), small instruments for space physics, in Proceedings of the Small Instrument Workshop, held 29-31 March, 1993 in Pasadena, CA, ed. by B.T. Tsurutani (National Aeronautics and Space Division, Washington, 1993), p. 7

A.C. Cummings, E.C. Stone, C.D. Steenberg, Astrophys. J. 578, 194 (2002)

M.I. Desai et al., Astrophys. J. 649, 470 (2006)

W. Dröge et al., Astrophys. J. 645, 1516 (2006)

J.R. Dwyer et al., Astrophys. J. 563, 403 (2001)

L.A. Fisk, B. Koslovsky, R. Ramaty, Astrophys. J. Lett. 190, L35 (1974)

A.B. Galvin et al., Space Sci. Rev. (2007, this issue)

J. Giacalone, J.R. Jokipii, J.E. Mazur, Astrophys. J. 532, L75 (2000)

G. Gloeckler, Space Sci. Rev. 89, 91 (1999)

D.K. Haggerty, E.C. Roelof, in 27th International Cosmic Ray Conference, ed. by W. Dröge, H. Kunow, M. Scholer (Schaltungsdienst Lange o.H.G., Berlin, 2001), p. 3238

D.K. Haggerty, E.C. Roelof, Astrophys. J. 579, 841 (2002)

E. Halpern, J.H. Marshall, IEEE Trans. Nucl. Sci. NS-15, 242 (1968)

E. Halpern, J.H. Marshall, D. Weeks, Nucleonics in Aerospace (Plenum, New York, 1968), p. 98

T.M. Harrington, J.H. Marshall, Rev. Sci. Instrum. 39, 184 (1968)

T.M. Harrington, J.H. Marshall, IEEE Trans. Nucl. Sci. NS-16, 314 (1969)

T.M. Harrington et al., Nucl. Instrum. Methods 118, 401 (1974)

G.C. Ho, E.C. Roelof, G.M. Mason, Astrophys. J. 621, L141 (2005)

J.L. Hoff, L.W. Townsend, J.W. Hines, IEEE Trans. Nucl. Sci. 50, 2296 (2003)

R.A. Howard et al., Space Sci. Rev. (2007, this issue)

J.F. Janni, Technical report No. AFWL-TR-65-150, Air Force Weapons Laboratory, Kirtland Air Force Base, NM, 1966

S.W. Kahler, A. Vourlidas, J. Geophys. Res. (2005). doi:10.1029/2006JA011073

A. Klassen et al., J. Geophys. Res. 110 (2005). CiteID A09S04

B. Klecker et al., Adv. Space Res. 38, 493 (2006)

S. Krucker, R.P. Lin, Astrophys. J. 542, L61 (2000)

A.W. Labrador et al., in 28th International Cosmic Ray Conference, ed. by T. Kajta, Y. Asaoka, A. Kawachi, Y. Matsubara, M. Sasaki (Universal Academy Press, Inc., Tokyo, 2003), p. 3269

A.W. Labrador et al., in 29th International Cosmic Ray Conference, vol. 1, ed. by B. Sripathi et al. (Tata Institute of Fundamental Research, Mumbai, 2005), p. 99

R.A. Leske et al., in Acceleration and Transport of Energetic Particles Observed in the Heliosphere, ed. by R.A. Mewaldt et al. (AIP, Melville, 2000), p. 293

R.A. Leske et al., in Connecting Sun and Heliosphere, ed. by B. Fleck, T.H. Zurbuchen, H. Lacoste (ESA, Whistler, 2003a), p. 616

R.A. Leske et al., in 28th International Cosmic Ray Conference, ed. by T. Kajta, Y. Asaoka, A. Kawachi, Y. Matsubara, M. Sasaki (Universal Academy Press, Inc., Tokyo, 2003b), p. 3253

R.A. Leske et al., Space Sci. Rev. (2007a). doi:10.1007/s11214-007-9191-5

R.A. Leske et al., Space Sci. Rev. (2007b). doi:10.1007/s11214-007-9185-3

G. Li, G.P. Zank, W.K.M. Rice, J. Geophys. Res. (2005). doi:10.1029/2004JA010600

R.P. Lin et al., Space Sci. Rev. (2007, this issue)

J.G. Luhmann et al., Adv. Space Res. 36, 1534 (2005)

J.G. Luhmann et al., Space Sci. Rev. (2007, this issue). doi:10.1007/s11214-007-9170-X

G.M. Mason, G. Gloeckler, D. Hovestadt, Astrophys. J. 280, 902 (1984)

G.M. Mason, J.E. Mazur, D.C. Hamilton, Astrophys. J. 425, 843 (1994)

G.M. Mason et al., Astrophys. J. 486, L149 (1997)

G.M. Mason et al., Geophys. Res. Lett. 26, 141 (1999)

G.M. Mason, J.R. Dwyer, J.E. Mazur, Astrophys. J. 545, L157 (2000)

G.M. Mason et al., Astrophys. J. 574, 1039 (2002)

G.M. Mason, J.E. Mazur, J.R. Dwyer, Astrophys. J. 565, L51 (2002)

G.M. Mason et al., Astrophys. J. 606, 555 (2004)

G.M. Mason et al., Space Sci. Rev. (2007, this issue). doi:10.1007/s11214-006-9087-9

J.E. Mazur et al., Geophys. Res. Lett. 26, 173 (1999)

J.E. Mazur et al., Astrophys. J. 532, L79 (2000)

J.E. Mazur, G.M. Mason, R.A. Mewaldt, Astrophys. J. 566, 555 (2002)

F.B. McDonald et al., in 29th International Cosmic Ray Conference, vol. 2, ed. by B. Sripathi Acharya et al. (Tata Institute of Fundamental Research, Mumbai, 2005), p. 35

D.J. McComas, N.A. Schwadron, Geophys. Res. Lett. (2006). doi:10.1029/2005GL025437 
M.A. McMahan, Nucl. Instr. Methods Phys. Res. B241, 409 (2005)

R.A. Mewaldt, in 26th International Cosmic Ray Conference, Invited, Rapporteur, and Highlight Papers, ed. by B.L. Dingus, D.B. Kieda, M.H. Salamon (AIP, Melville, 2000), p. 265

R.A. Mewaldt et al., in 28th International Cosmic Ray Conference, ed. by T. Kajta, Y. Asaoka, A. Kawachi, Y. Matsubara, M. Sasaki (Universal Academy Press, Inc., Tokyo, 2003), p. 3313

R.A. Mewaldt et al., in Proc. 29th International Cosmic Ray Conf., vol. 1, ed. by B. Sripathi Acharya et al. (Tata Institute of Fundamental Research, Mumbai, 2005a), p. 57

R.A. Mewaldt et al., J. Geophys. Res. (2005b). doi:10.1029/2005JA011038

R.A. Mewaldt et al., in The Physics of Collisionless Shocks, ed. by G. Li, G.P. Zank, C.T. Russell (AIP, Melville, 2005c), p. 227

R.A. Mewaldt et al., in Connecting Sun and Heliosphere, ed. by B. Fleck, T.H. Zurbuchen, H. Lacoste (ESA, Whistler, 2005d), p. 67

R.A. Mewaldt, C.M.S. Cohen, G.M. Mason, in Solar Eruptions and Energetic Particles, ed. by N. Gopalswamy, R.A. Mewaldt, J. Torsti (AGU, Washington, 2006), p. 115

R.A. Mewaldt, Space Sci. Rev. (2006). doi:10.1007/s11214-006-9091-0

R.A. Mewaldt et al., Space Sci. Rev. (2007). doi:10.1007/s11214-007-9200-8

E. Möbius et al., Geophys. Res. Lett. (2002). doi:10.1029/2001GL013410

H. Moraal et al., in Physics of the Heliosheath, ed. by J. Heerikhusen, V. Florinski, G.P. Zank, N.V. Pogorelov (AIP, Melville, 2006), p. 219

R. Müller-Mellin et al., Space Sci. Rev. (2007, this issue). doi:10.1007/s11214-007-9204-4

J.S. Neal, L.W. Townsend, Radiat. Prot. Dosim. 115, 38 (2005)

N.V. Nitta et al., Astrophys. J. (2006). doi:10.1086/507442

T.G. Onsager et al., in GOES-8 and Beyond, SPIE Conference Proceedings, ed. by E.R. Washwell (SPIE, Bellingham, 1996), pp. 281-290

M.E. Pesses, J.R. Jokipii, D. Eichler, Astrophys. J. Lett. 246, L85 (1981)

V.S. Ptuskin, Space Sci. Rev. 99, 281 (2001)

D.V. Reames, Adv. Space Res. 13, 331 (1993)

D.V. Reames, Adv. Space Res. 15, 41 (1995)

D.V. Reames, Space Sci. Rev. 90, 413 (1999)

D.V. Reames, C.K. Ng, Astrophys. J. 610, 510 (2004)

D.V. Reames, T.T. von Rosenvinge, R.P. Lin, Astrophys. J. 292, 716 (1985)

D.V. Reames, R.G. Stone, M.-B. Kallenrode, Astrophys. J. 380, 287 (1991)

D.V. Reames, J.-P. Meyer, T.T. von Rosenvinge, Astrophys. J. Suppl. 90, 649 (1994)

D.V. Reames et al., Geophys. Res. Lett. 24, 2917 (1997)

D.V. Reames, C.K. Ng, D. Berdichevsky, Astrophys. J. 550, 1064 (2001)

W.K.M. Rice, G.P. Zank, G. Li, J. Geophys. Res. (2003). doi:10.1029/2002JA009756

I.G. Richardson, Space Sci. Rev. 111, 267 (2004)

N.A. Schwadron et al., Geophys. Res. Lett. (2002). doi:10.1029/2002GL015829

R. Schwenn, E. Marsch, Physics of the Inner Heliosphere I and II (Springer, Berlin, 1991)

J.D. Slavin, P.C. Frisch, Astrophys. J. 565, 364 (2002)

E.C. Stone et al., Space Sci. Rev. 86, 1 (1998a)

E.C. Stone et al., Space Sci. Rev. 86, 357 (1998b)

E.C. Stone et al., Space Sci. Rev. 86, 285 (1998c)

E.C. Stone et al., Science 309, 2017 (2005)

R.E. Turner, in Solar Eruptions and Energetic Particles, ed. by N. Gopalswamy, A. Mewaldt, J. Torsti (AGU, Washington, 2006), p. 367

A.J. Tylka, W.F. Dietrich, P.R. Boberg, IEEE Trans. Nucl. Sci. 44, 2140 (1997)

A.J. Tylka et al., Astrophys. J. 558, L59 (2001)

A.J. Tylka et al., in 28th International Cosmic Ray Conference, ed. by T. Kajta, Y. Asaoka, A. Kawachi, Y. Matsubara, M. Sasaki (Universal Academy Press, Inc., Tokyo, 2003), p. 3305

A.J. Tylka et al., Astrophys. J. 625, 474 (2005)

J. Vandegriff et al., Adv. Space Res. 36, 2323 (2005)

T.T. von Rosenvinge et al., in Solar and Galactic Composition, ed. by R.F. Wimmerschweingruber (AIP, Melville, 2001), p. 343

T.T. von Rosenvinge et al., Space Sci. Rev. (2007, this issue)

A. Vourlidas et al., Astrophys. J. 534, 456 (2000)

Y.-M. Wang, M. Pick, G.M. Mason, Astrophys. J. 639, 495 (2006a)

L. Wang et al., Geophys. Res. Lett. (2006b). doi:10.1029/2005GL024434

M.E. Wiedenbeck et al., in 28th International Cosmic Ray Conference, ed. by T. Kajta, Y. Asaoka, A. Kawachi, Y. Matsubara, M. Sasaki (Universal Academy Press, Inc., Tokyo, 2003), p. 3245

M. Zhang et al., in Physics of the Heliosheath, ed. by J. Heerikhusen, V. Florinski, G.P. Zank, N.V. Pogorelov (AIP, Melville, 2006), p. 226

R.D. Zwickl et al., Space Sci. Rev. 86, 633 (1998) 